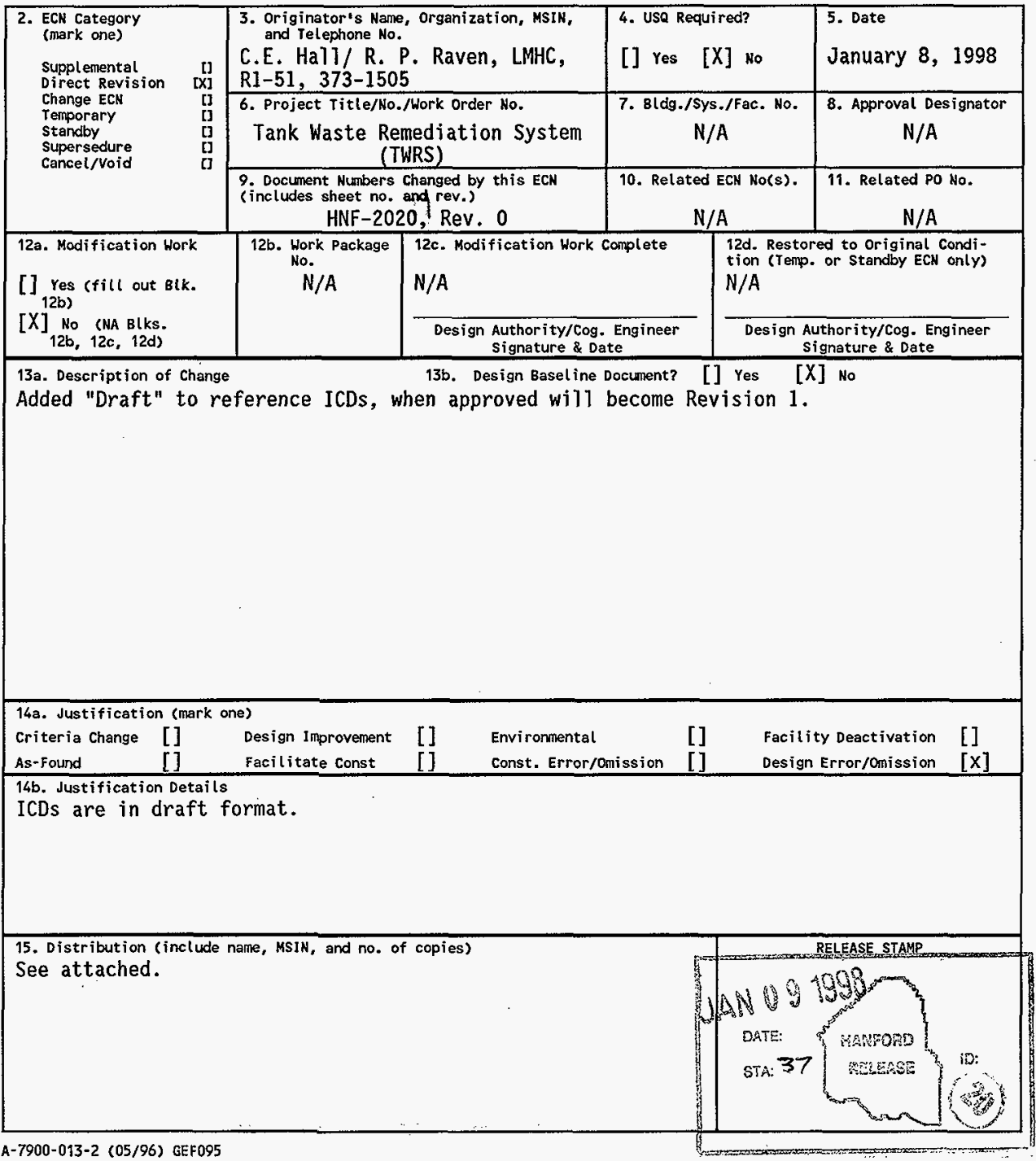




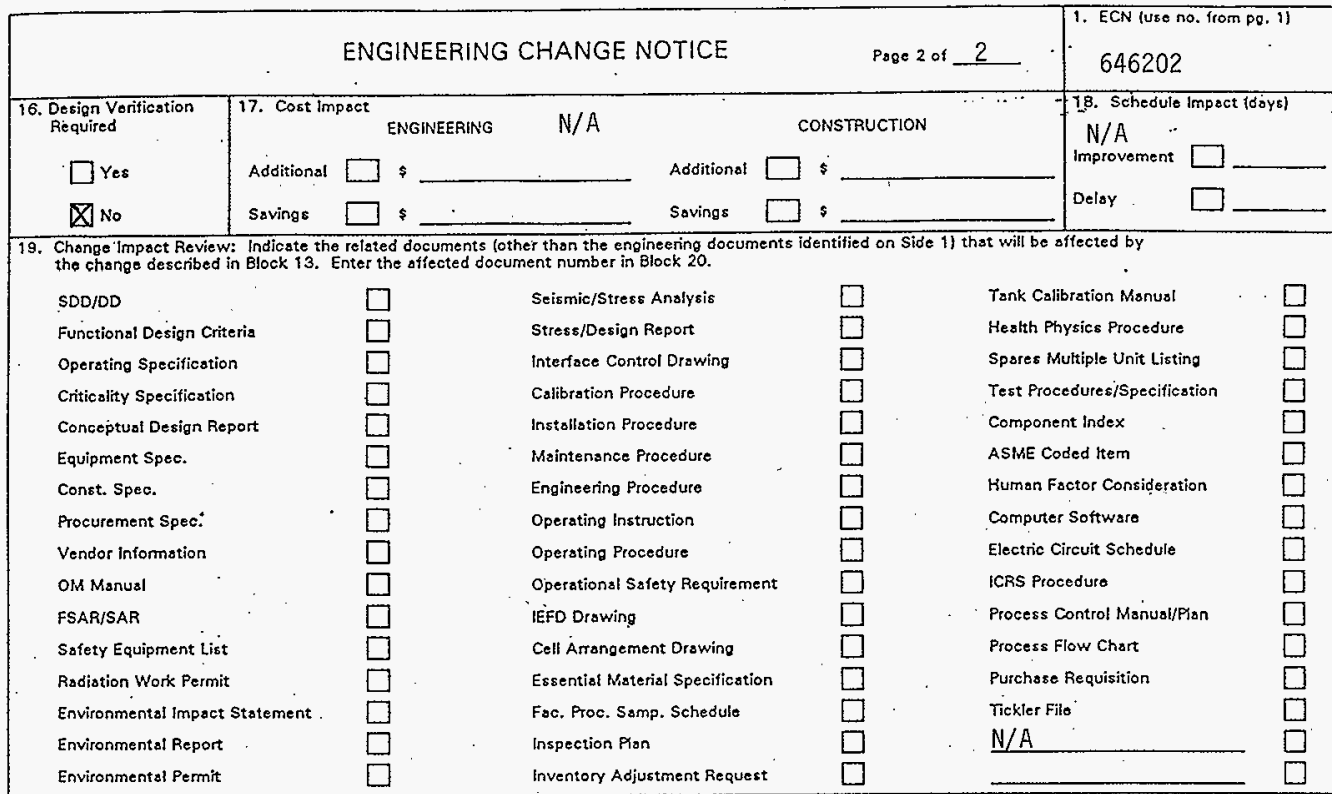

20. Other Affected Documents: (NOTE: Documents listed below will not be revised by this ECN.). Signatures below indicato that tho signing organization hes been notified of other sffected documents listed below.
$N / A$

Design Authority S.tt. Rifaey $1901 / 9 / 98$

Cog. Eng. $N / A$

Cog. Mor. N/A

QA

$$
N / A
$$

Sofety $N / A$

Environ. $N / A$

Ozher

Author, R.P. Raven Author's Mgr., R.D. Dhetanta
Dexign Agent

PE

aA

Sofety

Design

Environ.

Other

\section{DEPARTISENT OF ENERGY}

Signature or a Control Number that trocks the Approval Signature

ADDITIONAL 


\section{Tank Waste Remediation System Retrieval and Disposal Mission Readiness to Proceed Guidance and Requirements to Deliverables Crosswalk}

C. E. Hall / R. P. Raven

Lockheed Martin Hanford Company, Richland, WA 99352

U.S. Department of Energy Contract DE-AC06-96RL13200

EDT/ECN: $\quad 646202$

UC: 2030

Org Code: 7B220

Charge Code: D215P

B\&R Code: EW3130010 Total Pages: 84 noa

Key Words: TWRS, Retrieval and Disposal Mission; Readiness-to-Proceed

Abstract: Before RL can authorize proceeding with Phase 1B, the PHMC team must demonstrate its readiness to retrieve and deliver the waste to the private contractor(s) and to receive and dispose of the products and byproducts returned from the treatment. The PHMC team has organized their plans for providing these vitrification-support services into the Retrieval and Disposal Mission within the Tank Waste Remediation System (TWRS) Program.

TRADEMARK DISCLAIMER., Reference herein to any specific comercial product, process, or service by trade name, trademark, manufacturer, or otherwise, does not necessarily consti tute or imply its endorsement, recommendation, or favoring by the United States Government or any agency thereof or its contractors or subcontractors.

Printed in the United States of America. To obtain copies of this document, contact: WHC/BCS Document Control Services, P.O. Box 1970, Mailstop H6-08, Richland HA, 98352, Phone-1509) 372-2420Fax (509) 376-4989.
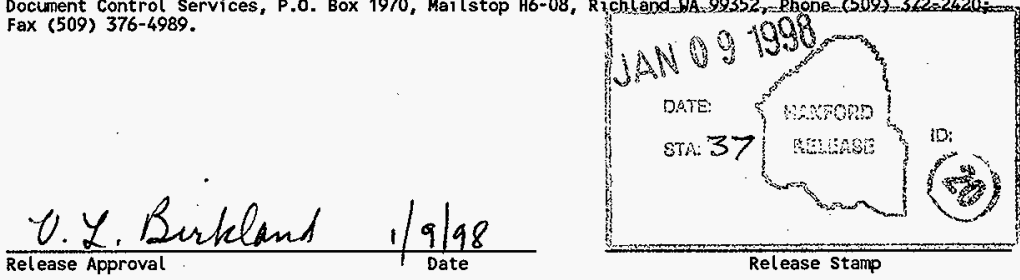

Release Stamp 


\section{RECORD OF REVISION}

(1) Document Number

HNF-2020

Page 1

(2) Title Tank Waste Remediation System Retrieval and Disposal Mission Readiness-to-

Proceed Guidance and Requirements to Deliverables Crosswalk

CHANGE CONTROL RECORO

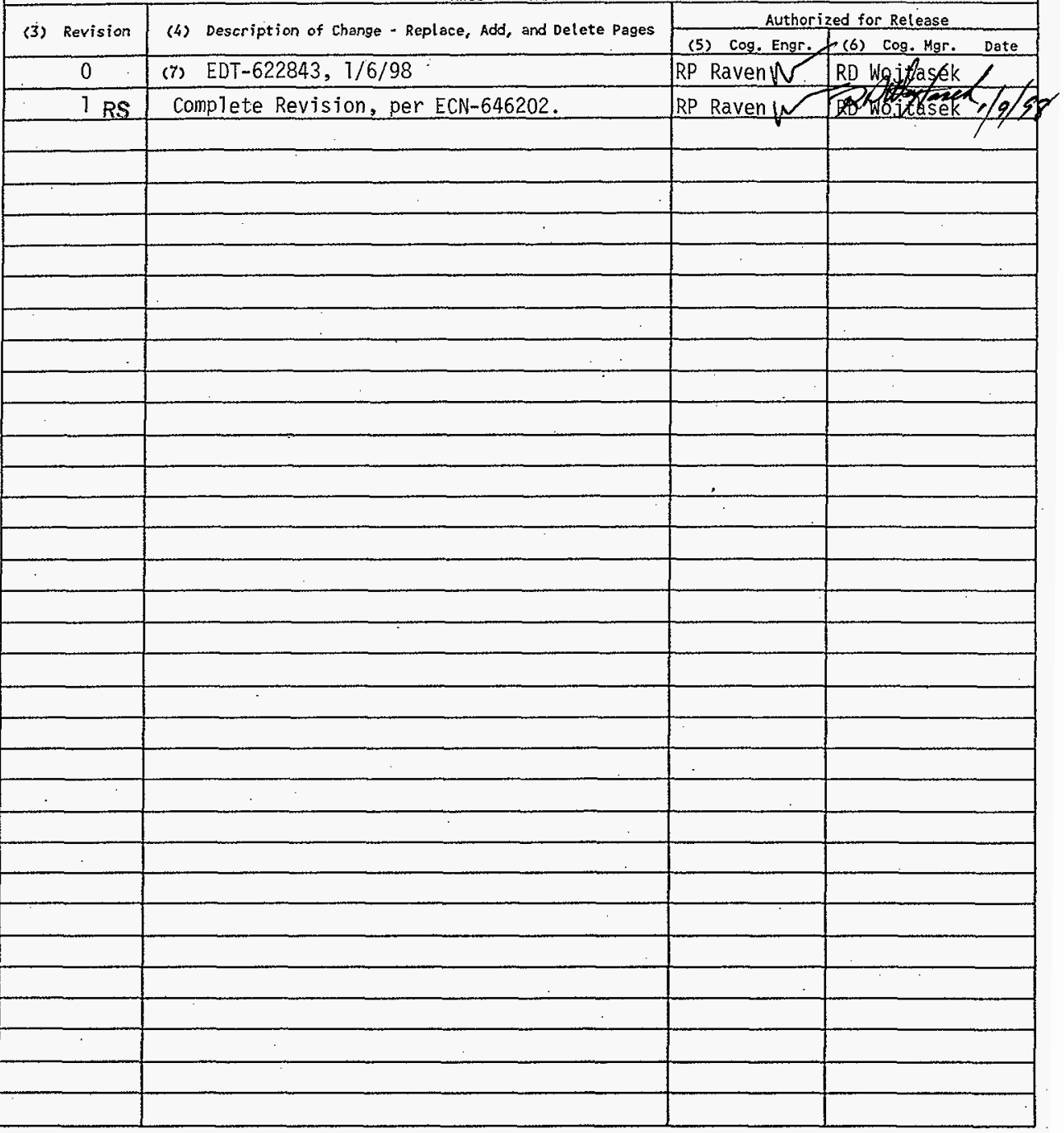


HNF-2020

Revision 1

\section{Tank Waste Remediation System Retrieval and Disposal Mission Readiness- to-Proceed Guidance and Requirements to Deliverables Crosswalk}

Prepared for the U.S. Department of Energy

$\$$ Fluor Daniel Hanford, Inc.

Hanford Management and Integration Contractor for the

U.S. Department of Energy under Contract DE-AC-0696-RLL13200 


\title{
Tank Waste Remediation System Retrieval and Disposal Mission Readiness-to-Proceed Guidance and Requirements to Deliverables Crosswalk
}

\author{
C. A. Hall
}

Dr. Charles Hall, Inc.

Date Published

January 1998

Prepared by Lockheed Martin Hanford Corporation

Richland, Washington

Prepared for the U.S. Department of Energy

Fluor Daniel Hanford, Inc.

P.O.: Box 1000

Richland, Washington

Hanford Management and Integration Contractor for the

U.S. Department of Energy under. Contract DE-AC-0696-RL13200 
HNF-2020 Rev 1

This page intentionally left blank. 
HNF-2020 Rev 1

Document Title: Tank Waste Remediation System Retrieval and Disposal Mission Readiness-to-Proceed Guidance and Requirements to Deliverables Crosswalk

Approved by:

D) N Weotome

D. M. McDaniel

Vice-President, IRM \& Systems Engineering and Integration Lockheed Martin Hanford Corporation 
HNF-2020 Rev 1

This page intentionally left blank. 


\section{CONTENTS}

A.1 RL letter 97-WDD-129, Contract Number DE-AC06-96RL13200, Tank Waste Remediation System (TWRS) Privatization - Hanford Contractors Readiness to Proceed ........................................... 3

A.2 RL letter 97-WDD-129, Contract Number DE-AC06-96RL13200, Tank Waste Remediation System (TWRS) Privatization - Hanford Contractors Readiness to Proceed ..............................................

A.3 RL letter 97-WDD-182, Review of Tank Waste Remediation System (TWRS) Privatization - Hanford Contractors Readiness to Proceed . .................. 3

B.1 DOE Order 430.1, Life Cycle Asset Management, GPG-FM-002, Critical Decision

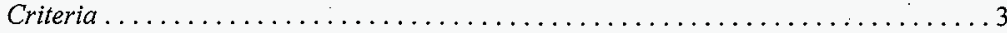

B.2. DOE Order 425.1, Startup and Restart of Nuclear Facilities, Section 4.d.(1)-(20) ...4 4

C.1 Draft Interface Control Documents $1-22 \ldots \ldots \ldots \ldots \ldots \ldots \ldots \ldots \ldots \ldots \ldots$

D.1 DOE Detailed Instructions for Assessment of RTP - Appendix C,

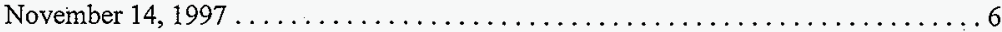

D.2 Plan for Determining the PHMC Team's RTP for Waste Feed Delivery $\ldots \ldots \ldots \ldots 6$

D.3 Plan for Determining the PHMC Team's RTP for Waste Feed Delivery.

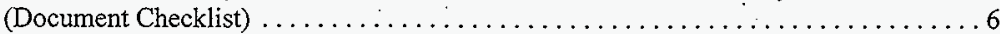

D.4 PHMC Team RTP Approach for the Evaluation of Administrative Readiness . . . . . 6

D.5 Draft Plan for Determining RTP for Infrastructure and Byproducts Delivery $\ldots \ldots \ldots 6$

D.6 Draft Plan for Determining RTP for Infrastructure and Byproducts Delivery, Appendix A, Technical Baseline Checklist $\ldots \ldots \ldots \ldots \ldots \ldots \ldots \ldots \ldots \ldots \ldots$.

D.7 Draft Plan for Determining RTP for Infrastructure and Byproducts Delivery, Appendix B, Programmatic Baseline Checklist $\ldots \ldots \ldots \ldots \ldots \ldots \ldots \ldots \ldots \ldots$

D.8 Draft Plan for Determining RTP for Infrastructure and Byproducts Delivery, Appendix C, Infrastructure (Management Baseline) Checklist . . . . . . . . . . . . 7

D.9 Draft Plan for Determining RTP for Infrastructure \& Byproducts Delivery, Appendix D, Feed Tank Transfer/Air Emissions (Technical Baseline) Checklist ..... 7 
D.10 Draft Plan for Determining RTP for Infrastructure and Byproducts Delivery, Appendix E, Infrastructure Feed Tank Transfer (Programmatic Baseline) Checklist . . . 7

D.11 Draft Plan for Determining RTP for Infrastructure and Byproducts Delivery, Appendix F, Infrastructure Feed Tank Transfer (Management Baseline) Checklist ... . 7

E.1 TWRS Waste Disposal Division Planning Guidance dated July $7,1997 \ldots \ldots \ldots \ldots 8$

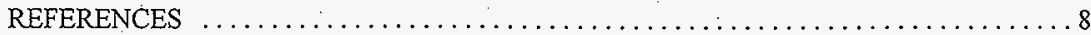

\section{LIST OF TABLES}

1. Guidance and Requirements to Deliverables Crosswalk 12

\section{LIST OF TERMS}

DOE

FDH

ICD

PHMC

RL

RTP

TWRS
U.S. Department of Energy

Fluor Daniel Hanford, Inc.

Interface Control Document

Project Hanford Management Contract

U.S. Department of Energy, Richland Operations Office readiness to proceed

Tank Waste Remediation System 


\section{TANK WASTE REMEDIATION SYSTEM RETRIEVAL AND DISPOSAL MISSION \\ READINESS-TO-PROCEED \\ GUIDANCE AND REQUIREMENTS TO DELIVERABLES CROSSWALK}

In September 1996, the U.S. Department of Energy, Richland Operations Office (RL) initiated the first of a two-phase program to remediate waste storage in tanks at the Hanford Site in Washington State. Initiating the first phase, RL signed contracts with two private companies who agreed to receive and vitrify a portion of the tank waste in a demonstration and to return the vitrified product and byproducts to the Project Management Hanford Contract (PHMC) team for disposition. The first phase of the overall remediation effort is a demonstration of treatment concepts, and the second phase includes treatment of the remaining tank wastes. The demonstration phase, Phase 1 of the project, is further subdivided into two parts, A and B. During Phase $1 \mathrm{~A}$, the vitrification contractors are to establish the technical, operational, regulatory, business, and financial elements required to provide treatment services on a fixedunit-price basis. Phase 1A deliverables will be evaluated by $\mathrm{RL}$ to determine whether it is in the best interest of the government to have one or more vitrification contractors proceed with Phase $1 \mathrm{~B}$, in which $6 \%$ to $13 \%$ of the tank waste would be treated in the demonstration. In addition, before RL can authorize proceeding with Phase 1B, the PHMC team must demonstrate its readiness to retrieve and deliver the waste to the private contractor(s) and to receive and dispose of the products and byproducts returned from the treatment. The PHMC team has organized their plans for providing these vitrification-support services into the Retrieval and Disposal Mission within the Tank Waste Remediation System (TWRS) Project.

Three RL core teams were established to assist in evaluating the PHMC team's readiness, specifically in regard to the following three task areas:

- Waste feed delivery

- Infrastructure and byproducts delivery

- Immobilized products.

The core teams each developed a set of criteria and plans to be used in evaluating the PHMC team's readiness to proceed (RTP). The three core teams are augmented by specialty teams established to provide expertise to the core team in the following five areas:

$\begin{array}{ll}\text { - } & \text { Risk management } \\ \text { - } & \text { Qufety } \\ \text { - } & \text { Admility assurance } \\ & \text { Regulatory compliance. }\end{array}$ 
The PHMC team evaluated its own readiness concurrent with an independent assessment of its readiness. Integral to the PHMC team's self-assessment, documents were identified that contain requirements and guidance for PHMC team's Retrieval and Disposal Mission. Some of these documents address portions of the overall TWRS Project that are applicable to the Retrieval and Disposal Mission. Because the parent TWRS Project is already underway, many of the activities needed to support vitrification operations are routine. In identifying evidence of readiness, the PHMC team identified, modified, or, when needed, developed documents at either the program or TWRS level, as appropriate. Proceeding with Phase 1B will employ proven technologies and expand the scope of a successfully operating program.

To facilitate self-assessment of RTP, the PHMC team identified requirements and guidance applicable to the initiation of the Retrieval and Disposal Mission field work. In the self-assessment, the PHMC team further identified which program or project document implements or responds to each guidance item or requirement. Because requirements and guidance are quite extensive, as are the number of program documents, the PHMC team developed a crosswalk linking each requirement or guidance item to one or more project or program planning documents. This crosswalk, or matrix, is included as Table 1. Following is an explanation of how items were selected from the applicable documents for inclusion in the matrix and how a graded level of detail was chosen for itemizing requirements and guidance from various source documents. The guidance/requirement source documents are organized into the following categories:

A. RL letters regarding PHMC team RTP

B. U.S. Department of Energy (DOE) Orders and Implementing Guides

C. Draft Interface Control Documents (ICD)

D. Draft Evaluation Guidance to the RL Core Teams (four documents)

E. RL Program Guidance.

The RL Core Team Critical Success Factors, dated December 22, 1997, are each addressed in Table 1, Section A-1, 36 Item Checklist, and Section B-2, DOE Order 425.1, Startup and Restart of Nuclear Facilities.

The RL Specialty Team Critical Success Factors dated November 22, 1997, are each addressed in Table 1, Section A-1, 36 Item Checklist; Section B-2, DOE Order 425.1; and numerous earlier DOE guidance documents.

Source document guidance and requirements are listed on the left side of the matrix. Numbers preceding guidance or requirements items are for internal use and have no other significance. Indented source document guidance or requirements indicate subordination. The program/project documents are shown on the top of the matrix. They are either deliverables to $\mathrm{RL}$ or supporting information available to RL upon request. Their titles are abbreviated in the matrix. 
An "I" is used to indicate where a program/project document addresses a specific requirement or guidance element. For simplification of the matrix, an "I," which is at the top level of a requirement or guidance element, also applies to all the subordinated items immediately below it. If a subordinate item has an "I," it only applies to that element.

\section{A.1 RL letter 97-WDD-129, Contract Number DE-AC06-96RL13200, Tank Waste Remediation System (TWRS) Privatization - Hanford Contractors Readiness to Proceed (36 Item Checklist)}

The RL's approach to evaluating the PHMC team's RTP is described in a letter from RL to Fluor Daniel Hanford, Inc. (FDH) (letter 97-WDD-129, Contract Number DE-AC0696RL13200, Tank Waste Remediation System (TWRS) Privatization - Hanford Contractors Readiness to Proceed [Taylor 1997a]). This letter includes the "Readiness-to-Proceed Evaluation Plan," which outlines RL's approach to evaluating the PHMC team's RTP. Each provision of the letter is itemized independently in the matrix, and it is shown where the evidence of response can be found. The first section in the matrix is composed of 36 items taken from the letter's Appendix B, "Checklist for PHMC Team Readiness to Proceed."

\section{A.2 RL letter 97-WDD-129, Contract Number DE-AC06-96RL13200, Tank Waste Remediation System (TWRS) Privatization - Hanford Contractors Readiness to Proceed (Body of Text)}

Requirements identified from the balance of the letter (Taylor 1997a) are included in the matrix as line items, and each is linked to one or more program/project planning documents.

\section{A.3 RL letter 97-WDD-182, Review of Tank Waste Remediation System (TWRS) Privatization - Hanford Contractors Readiness to Proceed}

The DOE's review of the PHMC team's RTP Plan is included in a letter from RL to FDH (letter 97-WDD-182, Review of Tank Waste Remediation System (TWRS) Privatization - Hanford Contractors Readiness to Proceed). Each provision of Taylor (1997b) is itemized independently in the matrix and cross-referenced to program or project plans.

\section{B.1 DOE Order 430.1, Life Cycle Asset Management, GPG-FM-002, Critical Decision Criteria}

DOE Order 430.1, Life Cycle Asset Management, as implemented through the "Good Practice Guide" GPG-FM-002, Critical Decision Criteria, was used extensively in determining which documents were needed for readiness at the Retrieval and Disposal Mission (i.e., the "program") level and which additional program documents were needed, or which program documents would require revision, at the TWRS level. For brevity, the provisions of the Good 
Practice Guide are included in the matrix at the summary outline level only, with notation corresponding to the specific provisions in the Good Practice Guide. Links are established to these general subject areas for cross-reference to program/project documents.

\section{B.2. DOE Order 425.1, Startup and Restart of Nuclear Facilities, Section 4.d.(1)-(20)}

The 20 core requirements of DOE Order 425.1 were employed as primary guidance in the development and revision of program/project documents. For brevity, 18 core requirements are listed and cross-referenced to program/project plans. Core requirements 16 and 20 were eliminated as applicable to DOE only.

\section{C.1 Draft Interface Control Documents 1-22}

The requirements extracted from the following 22 Draft ICDs include those commitments made by $R L$ for the project and/or program. These are treated as uncompromised requirements. The DOE commitments are itemized in their entirety:

- ICD-01, Interface Control Document Between DOE and the PHMC-Phase 1 Privatization-Raw Water (FDH 1997a)

- ICD-02, Interface Control Document Between DOE and the PHMC - Phase 1 Privatization-Potable Water (FDH 1997b)

- ICD-03, Interface Control Document Between DOE and the PHMC-Radioactive Solid Waste (FDH 1997c)

- ICD-04, Interface Between DOE and the PHMC - Dangerous Wastes (FDH 1997d)

- ICD-05, Interface Control Document Between DOE and the PHMC - NonRadioactive, Dangerous Liquid Effluent for Phase 1 Privatization (FDH 1997e)

- ICD-06, Interface Control Document Between DOE and the PHMC-Radioactive Dangerous Liquid Effluents (FDH 1997f)

- ICD-07, Interface Between DOE and the PHMC - Non-Dangerous Solid Water (FDH 1997g)

- ICD-08, Interface Between DOE and the PHMC - Liquid Sanitary Water (Not Released) (FDH 1997h)

- HNF-SP-1213-ICD-09, Interface Control Document Between DOE and the PHMC - Land for Siting Part A Privatization (FDH 1997i) 
- . HNF-SP-1214-ICD-10, Interface Control Document Between DOE and the PHMC - Deactivated Facility and Site for Phase 1 Privatization (FDH 1997j)

- ICD-11, Interface Control Document Between DOE and the PHMC - Electricity (FDH 1997k)

- ICD-12, Interface Control Document Between DOE and the PHMC - Phase I Privatization Roads and Rails (FDH 19971)

- ICD-13, Interface Control Document Between DOE and the PHMC - Phase I Privatization Non-Routine High-Level Solid Wastes (FDH 1997m)

- ICD-14, Interface Control Document Between DOE and the PHMC - Phase I Privatization Immobilized High-Level Waste Product (FDH 1997n)

- ICD-15, Interface Control Document Between DOE and the PHMC - Phase I Privatization Immobilized Low-Activity Waste Product (FDH 1997o)

- ICD-16, Interface Control Document Between DOE and the PHMC - Phase I Privatization Strontium/Transuranic/Entrained Solids (FDH 1997p)

- ICD-17, Interface Control Document Between DOE and the PHMC - Phase I Privatization Cesium-137 Intermediate Product (FDH 1997q)

- ICD-18, Interface Control Document Between DOE and the PHMC-Phase I Privatization - ${ }^{99}$ Technetium Secondary Product (FDH 1997r)

- ICD-19, Interface Control Document Between DOE and the PHMC - LowActivity Waste Feed (FDH 1997s)

- ICD-20, Interface Control Document Between DOE and the PHMC - Phase I Privatization - High-Level Waste Feed (FDH 1997t)

- ICD-21, Interface Control Document Between DOE and the PHMC - Phase 1 Waste Feed Tank Support Systems Low-Activity Waste Product (FDH 1997u)

- ICD-22; Interface Control Document Between DOE and the PHMC - Air Emissions (FDH 1997v). 


\section{D.1 DOE Detailed Instructions for Assessment of RTP - Appendix C, November 14, 1997}

Applicable criteria were identified from this draft guidance to the DOE core teams and specialty teams (RL 1997). These criteria are itemized in detail for cross-reference to the documents.

\section{D.2 Plan for Determining the PHMC Team's RTP for Waste Feed Delivery}

Applicable criteria were identified from the body of this draft guidance to the DOE Waste Feed Delivery review team. These criteria are itemized in detail for cross-reference to the planning documents.

\section{D.3 Plan for Determining the PHMC Team's RTP for Waste Feed Delivery (Document Checklist)}

Criteria were derived from Table 1 of the DOE Waste Feed Delivery core team's plan (Potter and Treat 1998), which provided examples of what deliverables could be expected from the PHMC team. Because the document titles are draft and are not always precise, and because some of the documents listed could be developed at later stages of the project, the titles are listed and cross-referenced by content to the actual program/project documents.

\section{D.4 PHMC Team RTP Approach for the Evaluation of Administrative Readiness}

This DOE document is early-draft planning guidance to its core team. The document was provided to the PHMC team with a caution to expect revision. Therefor, criteria items were selected at the highest level (i.e., those that were considered standards). These summary-level standards are incorporated as line items in the matrix and cross-referenced to program/project planning documents.

\section{D.5 Draft Plan for Determining RTP for Infrastructure and Byproducts Delivery}

This DOE draft document outlines criteria for its review teams and includes the checklist in D.6 through D.11 as well as some guidance text. This document is instructions for the DOE review of the RTP deliverables. 
D.6 Draft.Plan for Determining RTP for Infrastructure and Byproducts Delivery, Appendix A, Technical Baseline Checklist

This source document includes checklists derived from the 36 criteria found in Taylor (1997a). Because the 36 criteria were covered elsewhere in the matrix, restatement of the criteria or listing the items that obviously follow from the 36 criteria was avoided. Instead, checklist items that might be overlooked when using Taylor (1997a) directly are listed in the matrix. (See D.5 for explanation.)

D.7 : Draft Plan for Determining RTP for Infrastructure and Byproducts Delivery, Appendix B, Programmatic Baseline Checklist

Checklist items that might be overlooked when using Taylor (1997a) directly are listed in the matrix. (See D.5 for explanation.)

D.8 Draft Plan for Determining RTP for Infrastructure and Byproducts Delivery, Appendix C, Infrastructure (Management Baseline) Checklist

Checklist items that might be overlooked when using Taylor (1997a) directly are listed in the matrix. (See D.5 for explanation.)

D.9 Draft Plan for Determining RTP for Infrastructure \& Byproducts Delivery, Appendix D, Feed Tank Transfer/Air Emissions (Technical Baseline) Checklist

Checklist items that might be overlooked when using Taylor (1997a) directly are listed in the matrix. (See D.5 for explanation.)

D.10. Draft Plan for Determining RTP for Infrastructure and Byproducts Delivery, Appendix E, Infrastructure Feed Tank Transfer (Programmatic Baseline) Checklist

Checklist items that might be overlooked when using Taylor (1997a) directly are listed in the matrix. (See D.5 for explanation.)

D.11 Draft Plan for Determining RTP for Infrastructure and Byproducts Delivery, Appendix F, Infrastructure Feed Tank Transfer (Management Baseline) Checklist

Checklist items are listed in the matrix that might be overlooked when using Taylor (1997a) directly. (See D.5 for explanation.) 
HNF-2020 Rev 1

\section{E.1 TWRS Waste Disposal Division Planning Guidance dated July 7, 1997}

The TWRS planning guidance was screened for applicability because it is a TWRS Project-wide document. Portions selected as directly or indirectly applicable to the Retrieval and Disposal Mission were listed, summarized, and interpreted as nonrequirement guidance only. These criteria should include those found in the Draft Plan for Determining.RTP for Infrastructure \& Byproducts Delivery, Appendix G, Planning Guidance; therefor, Appendix G criteria were not itemized into the matrix.

\section{REFERENCES}

\section{U. S. Department of Energy Good Practice Guides}

GPG-FM-002, Critical Decision Criteria, Life Cycle Asset Management, Good Practice Guide, U.S. Department of Energy, Washington, D.C.

\section{U.S. Department of Energy Orders}

DOE Order 425:1, Startup and Restart of Nuclear Facilities, U.S. Department of Energy, Washington, D.C.

DOE Order 430.1, Life Cycle Asset Management, U.S. Department of Energy, Washington, D.C.

\section{Documents}

FDH, 1997a, Interface Control Document Between DOE and the PHMC - Phase 1 PrivatizationRaw Water, HNF-SP-1208-ICD-01, DRAFT prepared by Lockheed Martin Hanford Corporation for Fluor Daniel Hanford, Inc., Richland, Washington.

FDH, 1997b, Interface Control Document Between DOE and the PHMC - Phase I PrivatizationPotable Water, HNF-SP-1208-ICD-02, DRAFT prepared by Lockheed Martin Hanford Corporation for Fluor Daniel Hanford, Inc., Richland, Washington.

FDH, 1997c, Interface Control Document Between DOE and the PHMC - Radioactive Solid Waste, HNF-SP-1209-ICD-03, DRAFT prepared by Lockheed Martin Hanford Corporation for Fluor Daniel Hanford, Inc., Richland, Washington.

FDH, 1997d, Interface Between DOE and the PHMC - Dangerous Wastes, ICD-04, prepared by Lockheed Martin Hanford Corporation for Fluor Daniel Hanford, Inc., Richland, Washington (not released). 
FDH, 1997g, Interface Between DOE and the PHMC - Non-Dangerous Solid Waste, ICD-07 prepared by Lockheed Martin Hanford Corporation for Fluor Daniel Hanford, Inc., Richland, Washington (not released).

FDH, 1997h, Interface Between DOE and the PHMC - Interface Between DOE and the PHMC - Liquid Sanitary Water, ICD-08, prepared by Lockheed Martin Hanford Corporation for Fluor Daniel Hanford, Inc., Richland, Washington (not released).

FDH, 1997i, Interface Control Document Between DOE and the PHMC - Land for Siting Part A Privatization, HNF-SP-1215-ICD-09, DRAFT prepared by Lockheed Martin Hanford Corporation for Fluor Daniel Hanford, Inc., Richland, Washington.

FDH, 1997j, Interface Control Document Between DOE and the PHMC - Deactivated Facility and Site for Phase 1 Privatization, HNF-SP-1216-ICD-10, DRAFT prepared by Lockheed Martin Hanford Corporation for Fluor Daniel Hanford, Inc., Richland, Washington.

FDH, 1997k, Interface Control Document Between DOE and the PHMC - Electricity, HNF-SP-1217-ICD-11, DRAFT prepared by Lockheed Martin Hanford Corporation for Fluor Daniel Hanford, Inc., Richland, Washington.

FDH, 1997l, Interface Control Document Between DOE and the PHMC - Phase 1 Privatization Roads and Rails, HNF-SP-1218-ICD-12, DRAFT prepared by Lockheed Martin Hanford Corporation for Fluor Daniel Hanford, Inc., Richland, Washington.

FDH, $1997 \mathrm{~m}$, Interface Control Document Between DOE and the PHMC - Phase I Privatization Non-Routine High-Level Solid Wastes, HNF-SP-1219-ICD-13, DRAFT prepared by. Lockheed Martin Hanford Corporation for Fluor Daniel Hanford, Inc., Richland, Washington.

FDH, 1997n, Interface Control Document Between DOE and the PHMC - Phase 1 Privatization Immobilized High-Level Waste Product, HNF-SP-1220-ICD-14, DRAFT prepared by Lockheed Martin Hanford Corporation for Fluor Daniel Hanford, Inc., Richland, Washington.

FDH, 1997o, Interface Control Document Between DOE and the PHMC - Phase 1 Privatization Immobilized Low-Activity Waste Product, HNF-SP-1221-ICD-15, DRAFT prepared by Lockheed Martin Hanford Corporation for Fluor Daniel Hanford, Inc., Richland, Washington.

FDH, 1997p, Interface Control Document Between DOE and the PHMC - Phase 1 Privatization Strontium/Transuranic/Entrained Solids, HNF-SP-1222-ICD-16, DRAFT prepared by Lockheed Martin Hanford Corporation for Fluor Daniel Hanford, Inc:, Richland, Washington. 
FDH, 1997o, Interface Control Document Between DOE and the PHMC - Phase 1 Privatization Immobilized Low-Activity Waste Product, HNF-SP-1221-ICD-15, DRAFT prepared by Lockheed Martin Hanford Corporation for Fluor Daniel Hanford, Inc., Richland, Washington.

FDH, 1997p, Interface Control Document Between DOE and the PHMC - Phase 1 Privatization Strontium/Transuranic/Entrained Solids, HNF-SP-1222-ICD-16, DRAFT prepared by Lockheed Martin Hanford Corporation for Fluor Daniel Hanford, Inc., Richland, Washington.

FDH, 1997q, Interface Control Document Between DOE and the PHMC - Phase 1 Privatization Cesium-137 Intermediate Product, HNF-SP-1223-ICD-17, DRAFT prepared by Lockheed Martin Hanford Corporation for Fluor Daniel Hanford, Inc., Richland, Washington.

FDH, 1997r, Interface Control Document Between DOE and the PHMC - Phase 1 Privatization ${ }^{99}$ Technetium Secondary Product, HNF-SP-1224-ICD-18, DRAFT prepared by Lockheed Martin Hanford Corporation for Fluor Daniel Hanford, Inc., Richland, Washington.

FDH, 1997s, Interface Control Document Between DOE and the PHMC - Low-Activity Waste Feed, HNF-SP-1225-ICD-19, DRAFT prepared by Lockheed Martin Hanford Corporation for Fluor Daniel Hanford, Inc., Richland, Washington.

FDH, 1997t, Interface Control Document Between DOE and the PHMC - Phase 1 Privatization High-Level Waste Feed, HNF-SP-1224-ICD-20, DRAFT prepared by Lockheed Martin Hanford Corporation for Fluor Daniel Hanford, Inc., Richland, Washington.

FDH, 1997u, Interface Control Document Between DOE and the PHMC - Phase 1 Waste Feed Tank Support Systems Low-Activity Waste Product, HNF-SP-1225-ICD-21, DRAFT prepared by Lockheed Martin Hanford Corporation for Fluor Daniel Hanford, Inc., Richland, Washington.

FDH, 1997v, Interface Control Document Between DOE and the PHMC - Air Emissions, HNF-SP-1225-ICD-22, DRAFT prepared by Lockheed Martin Hanford Corporation for Fluor Daniel Hanford, Inc., Richland, Washington.

Potter, R. D., and R. L. Treat, 1998, Tank Waste Remediation System Retrieval and Disposal Mission Waste Feed Delivery Plan, HNF-1881, Rev. 1, prepared by Lockheed Martin Hanford Corporation for Fluor Daniel Hanford, Inc., Richland, Washington.

RL, 1997, Detailed Instructions for Assessment of RTP, Informal Guidance Documents, U.S. Department of Energy, Richland Operations Office, Richland, Washington. 
Taylor, W. J., 1997a, Contract Number DE-AC06-96RL13200, Tank Waste Remediation System (TWRS) Privatization - Hanford Contractors Readiness to Proceed (letter 97-WDD-129 to H. J. Hatch, Fluor Daniel Hanford, Inc., August 8), U.S. Department of Energy, Richland Operations Office, Richland, Washington.

Taylor, W. J., 1997b, Review of Tank Water Remediation System (TWRS) PrivatizationHanford Contractors Readiness to Proceed, (letter 97-WDD-182 to H. J. Hatch, Fluor Daniel Hanford, Inc., December 2), U.S. Department of Energy, Richland Operations Office, Richland, Washington. 
HNF-2020 Rev 1

Table 1. Guidance and Requirements to Deliverables Crosswalk.

["Many" 11" x 17" pages to follow.] 


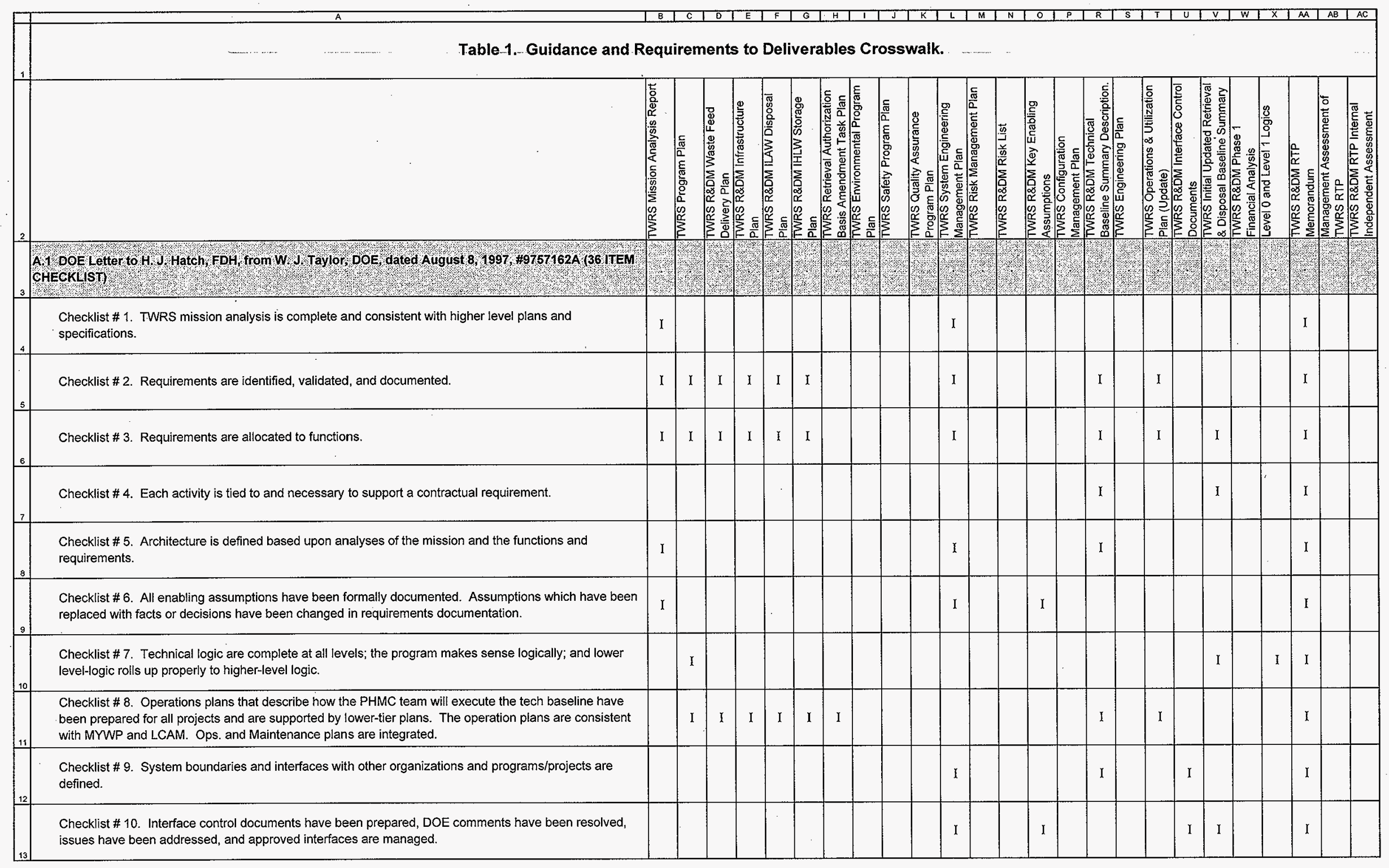




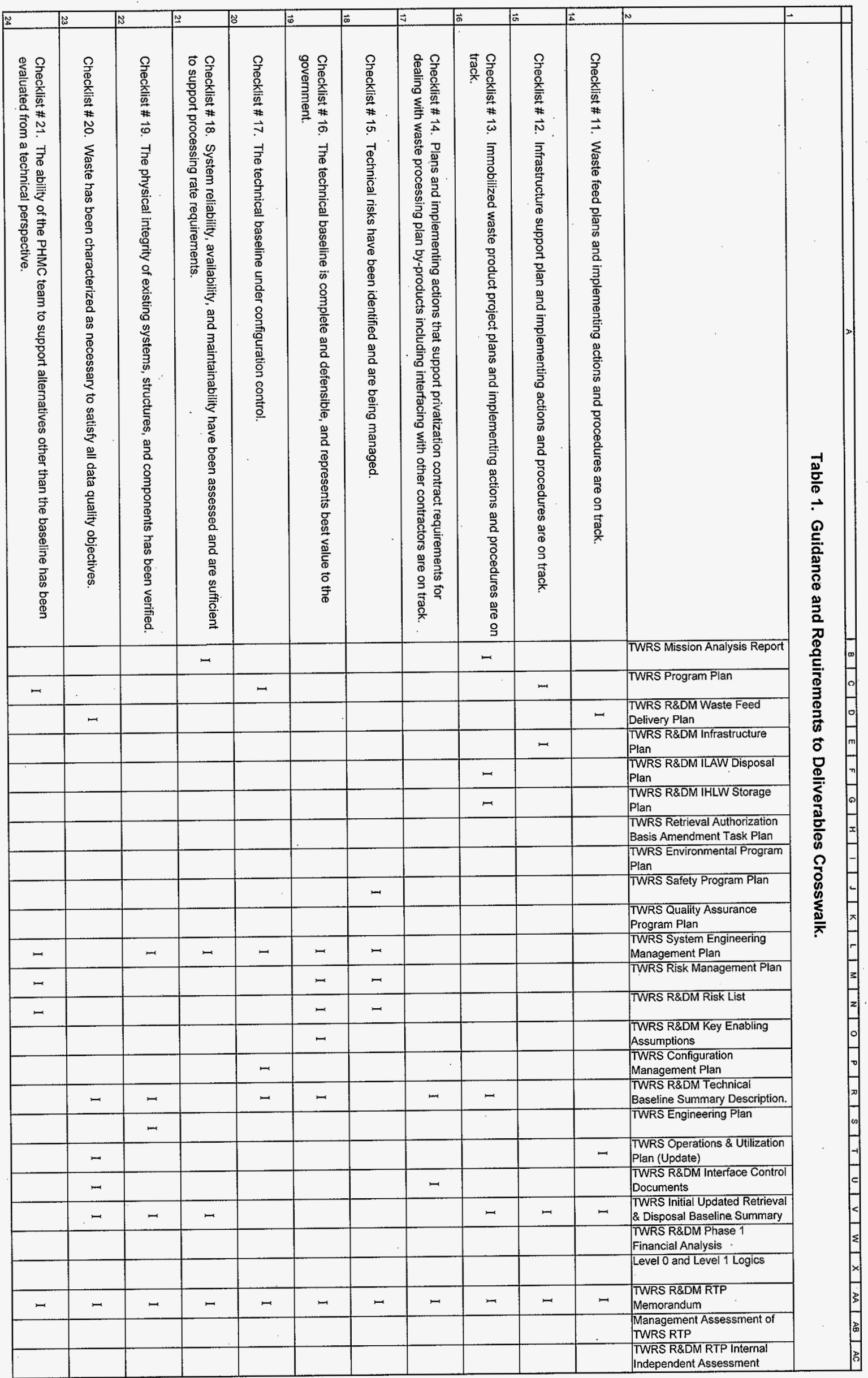





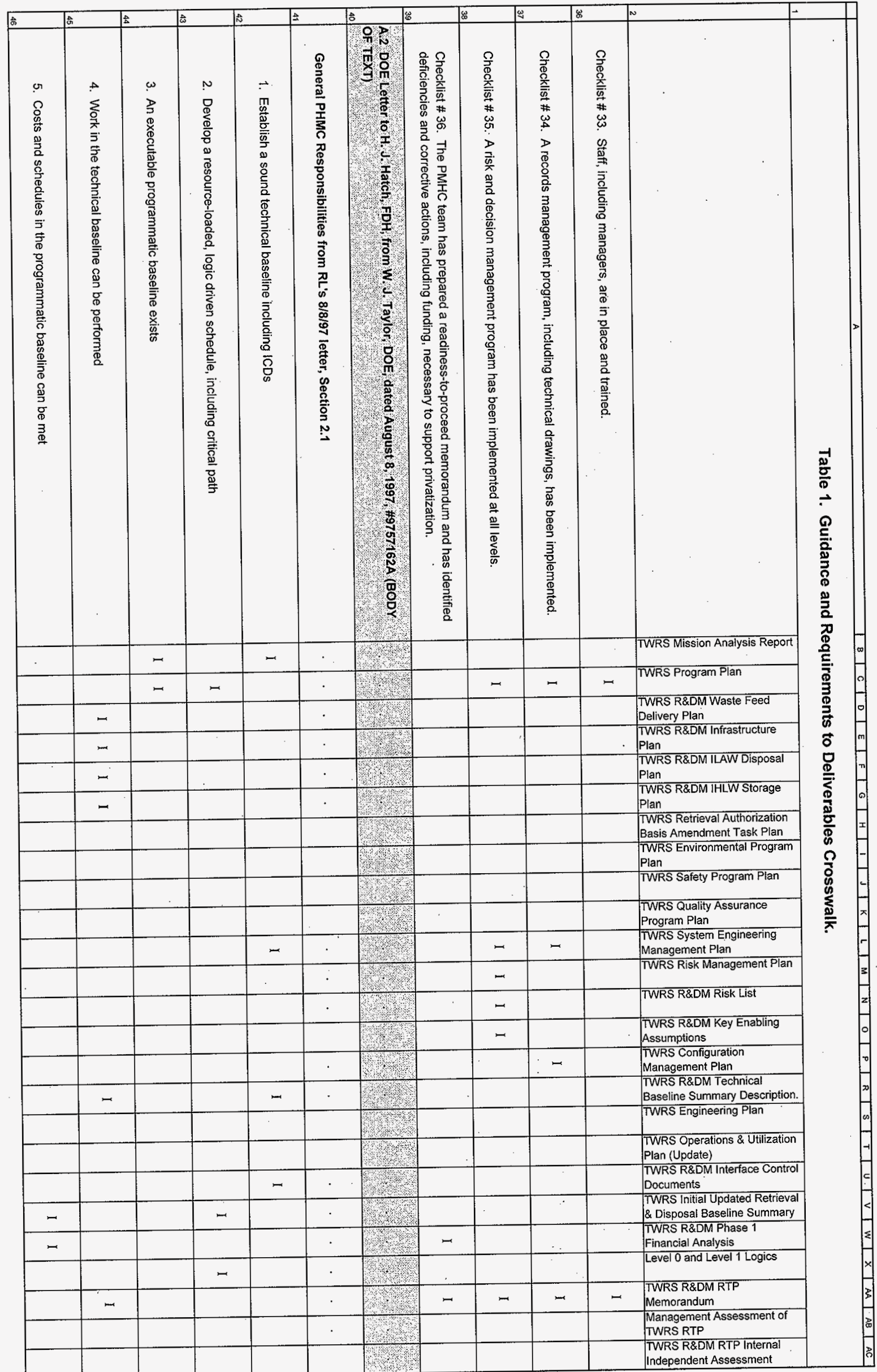




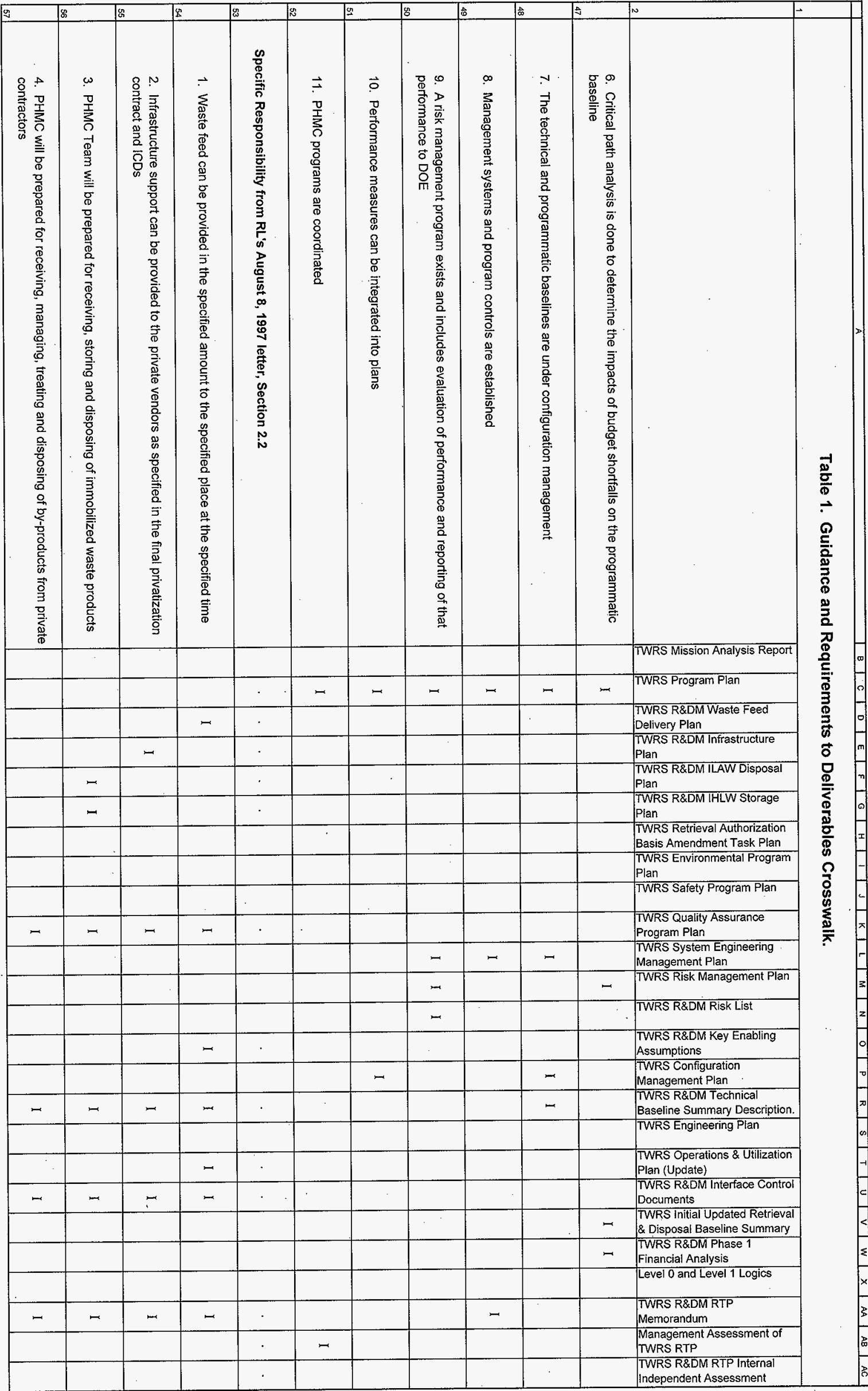




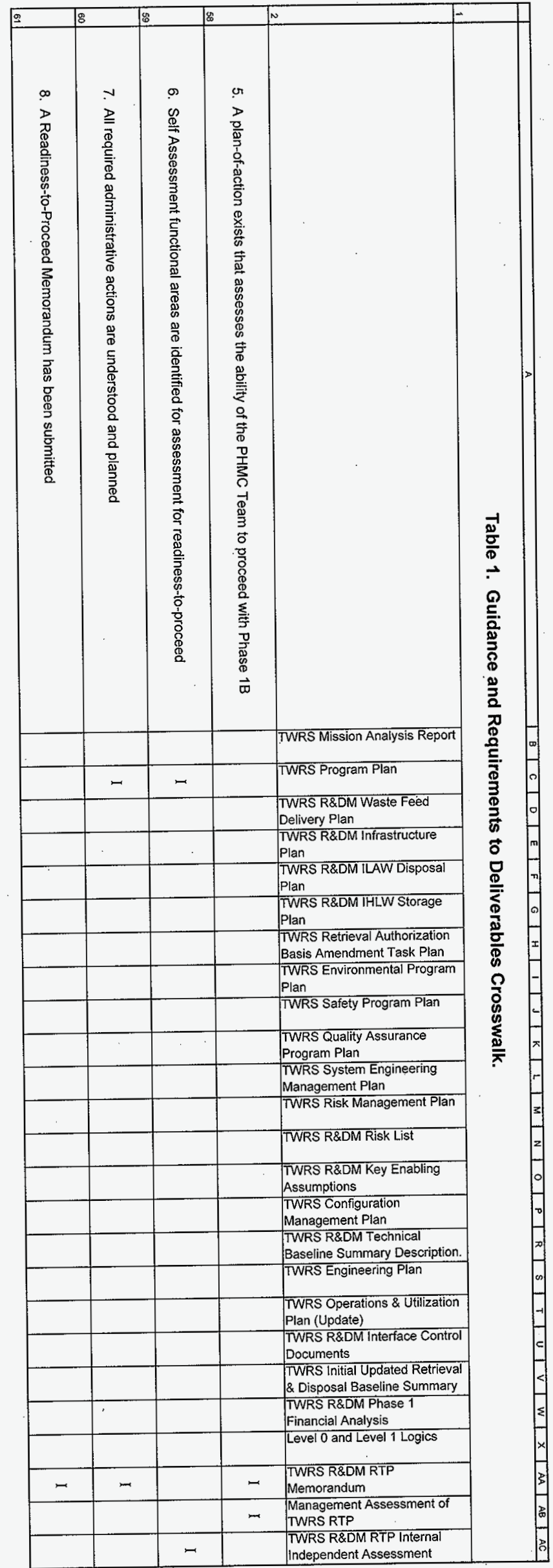




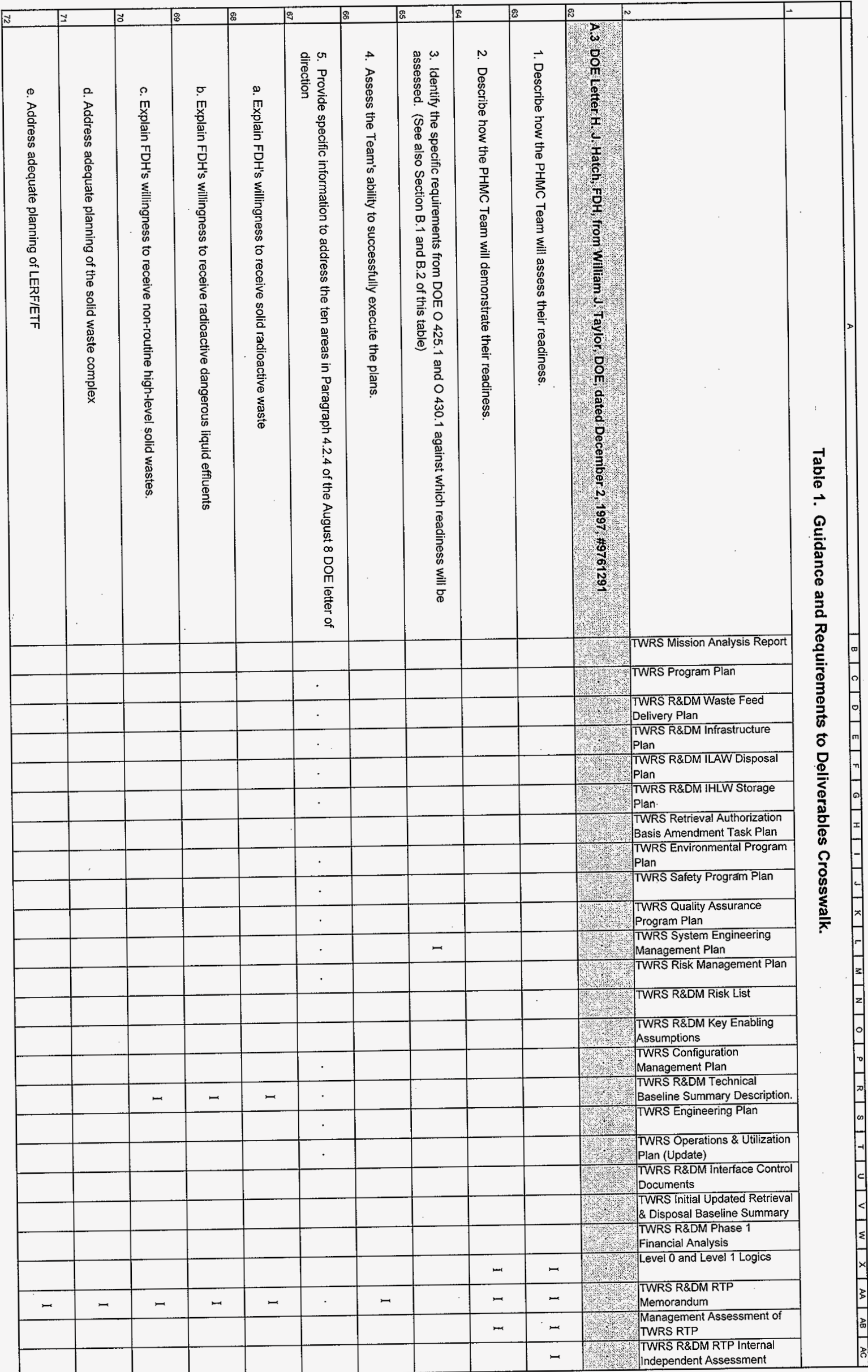




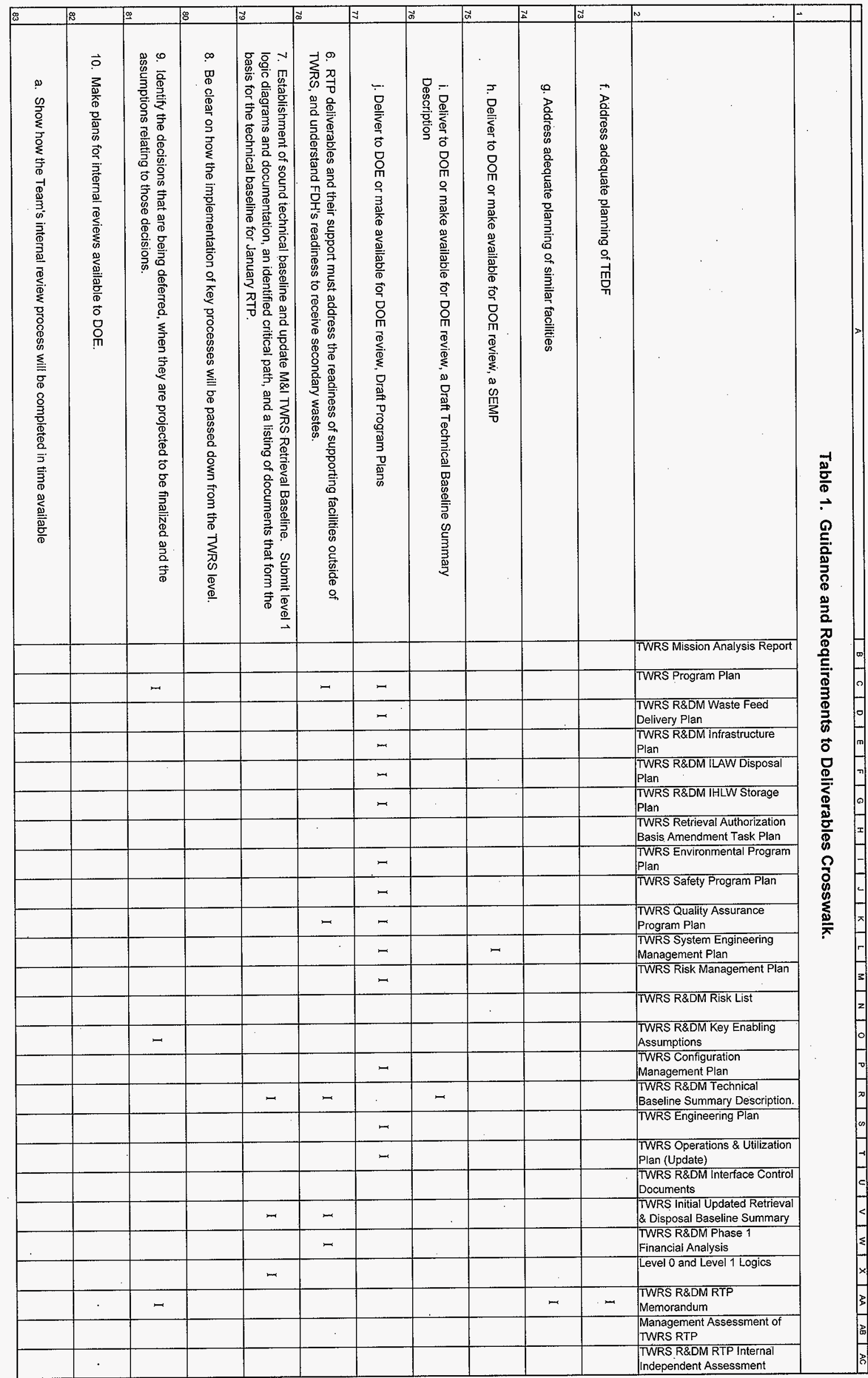




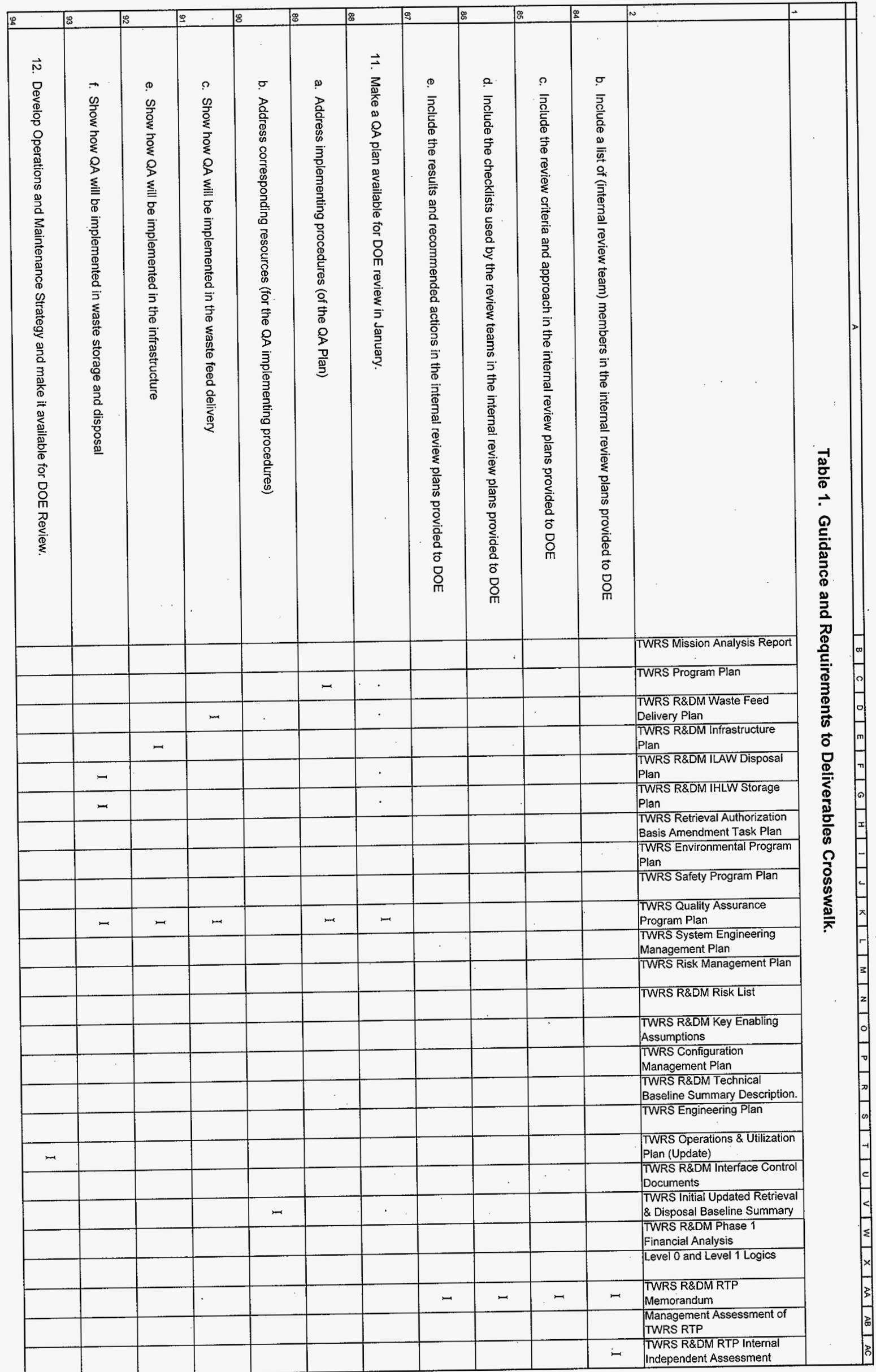




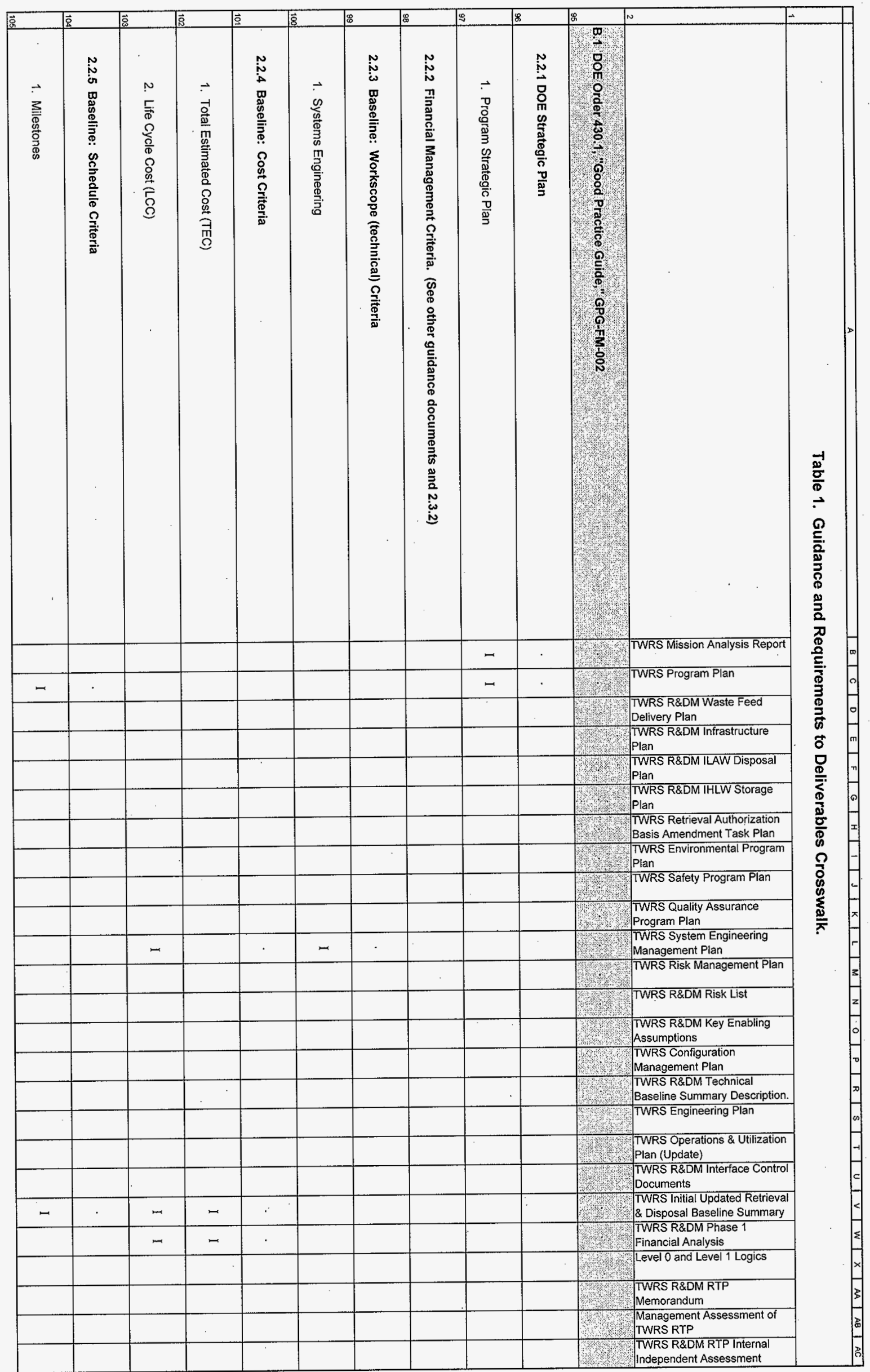




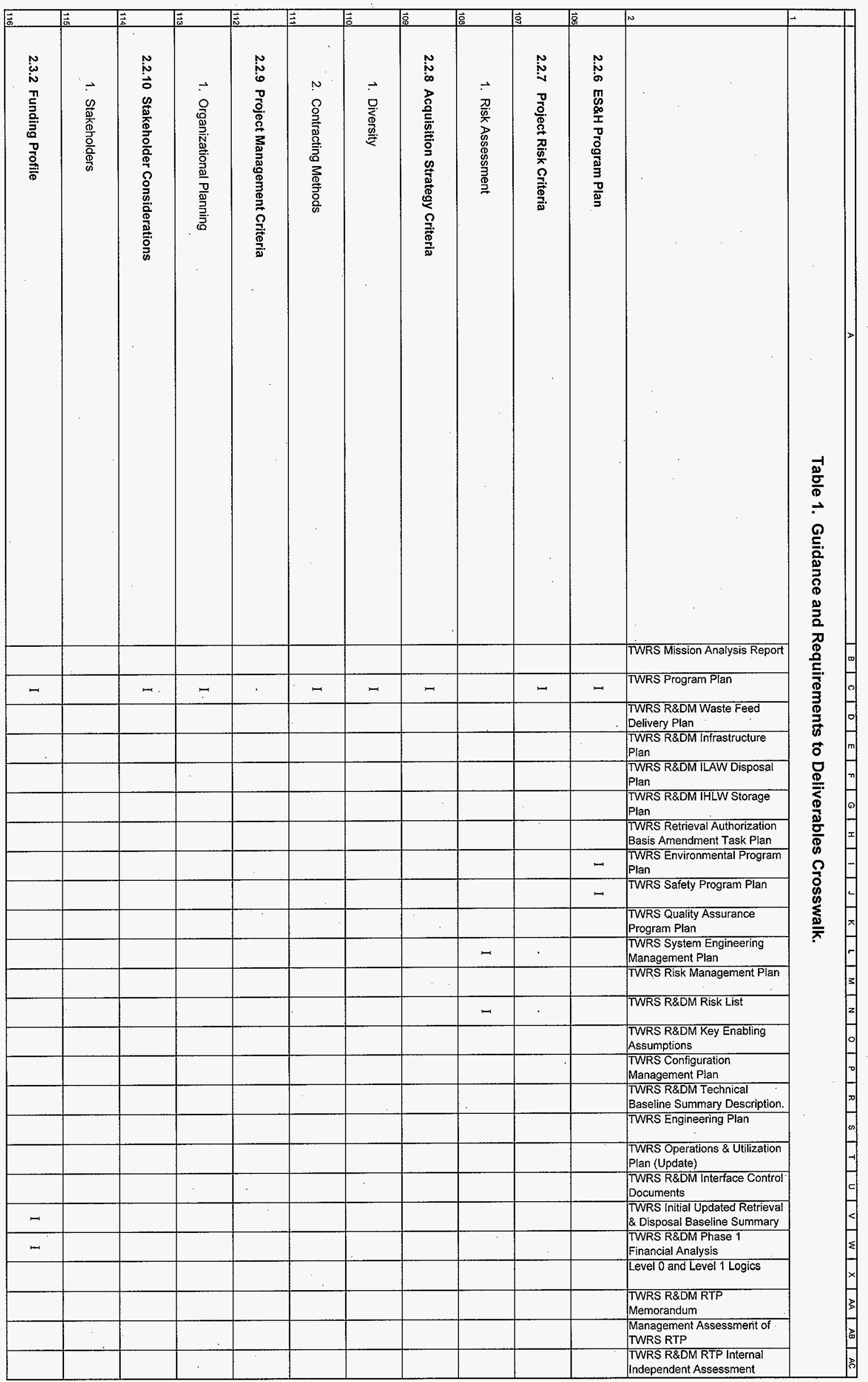




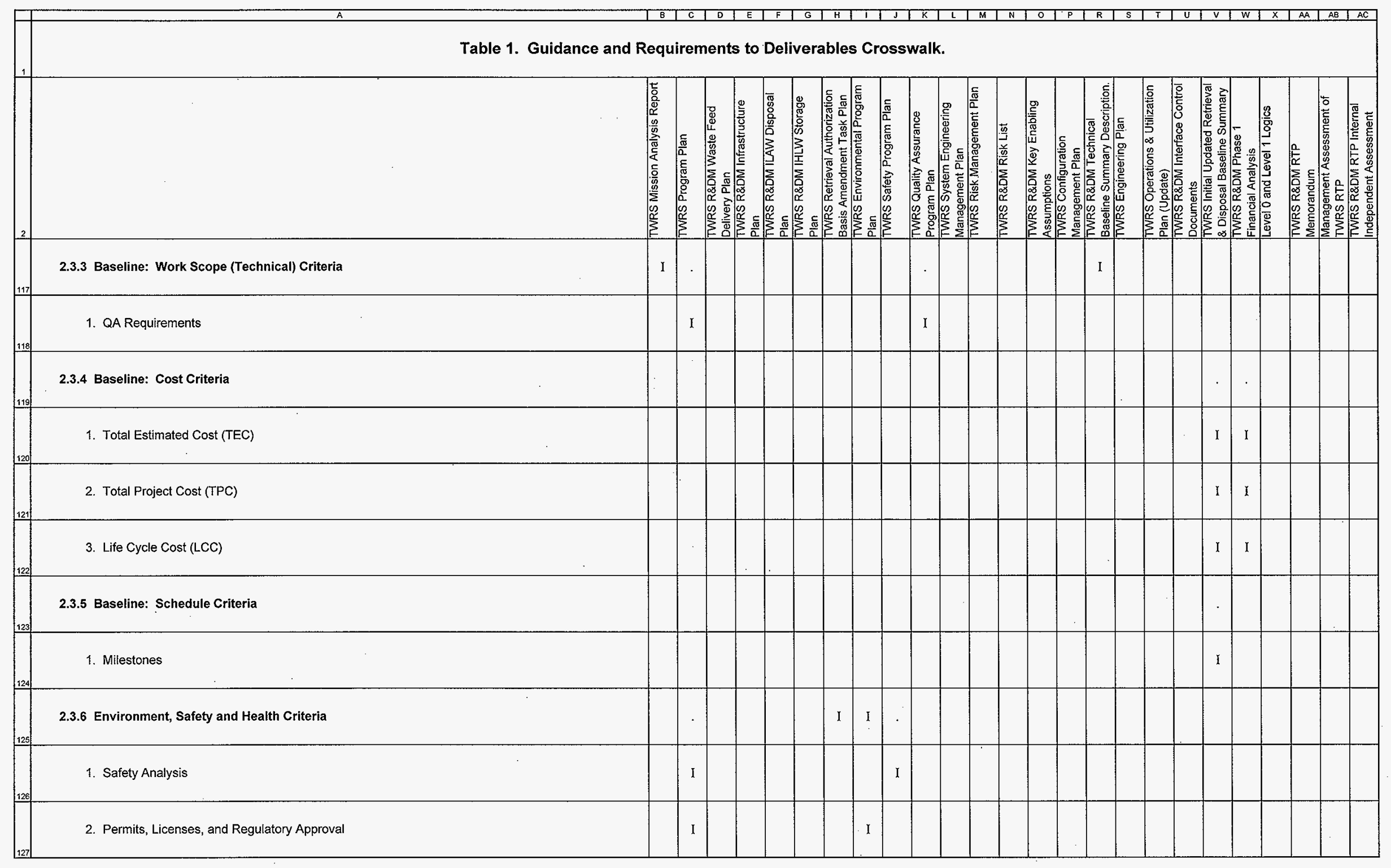




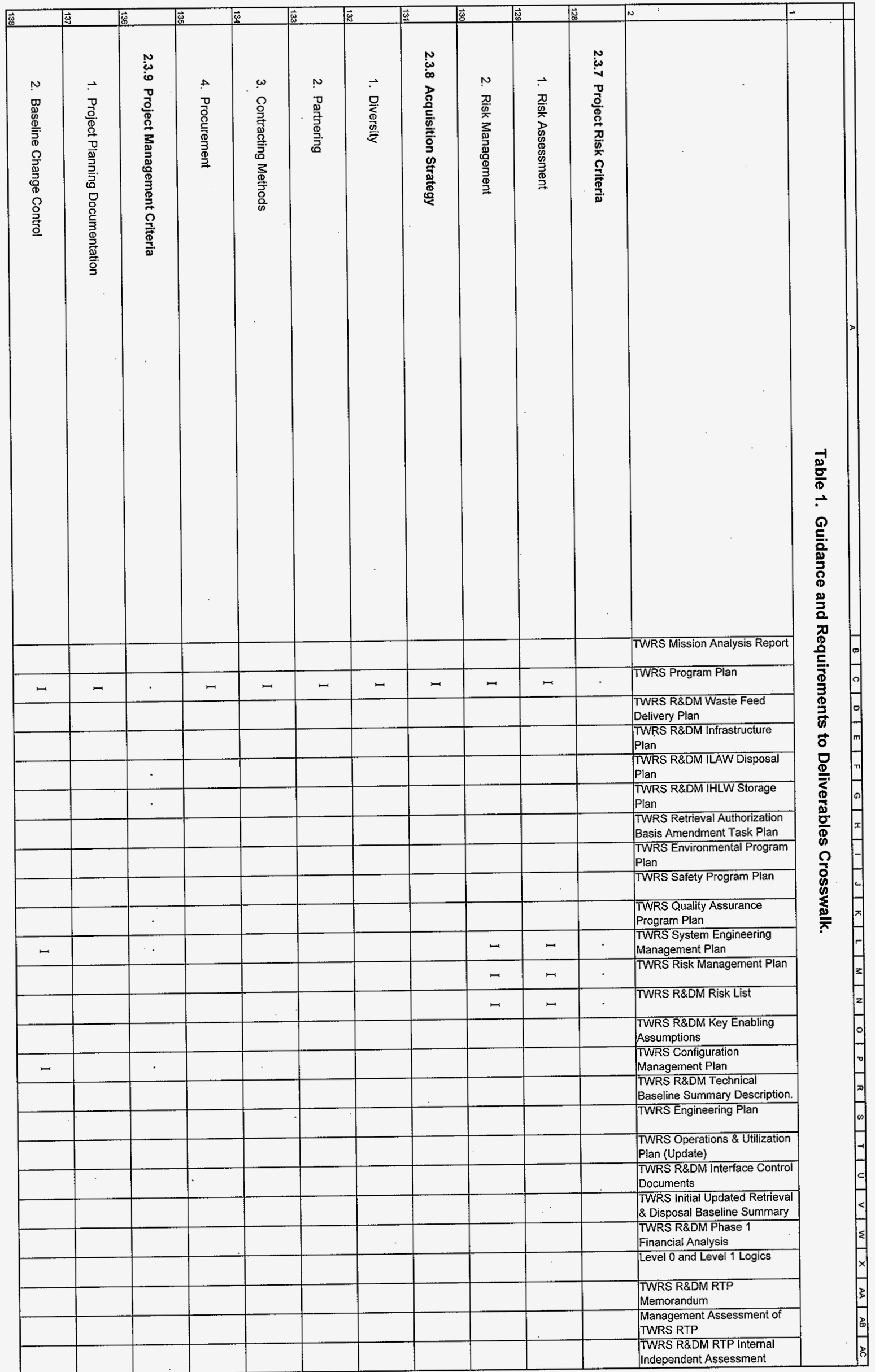




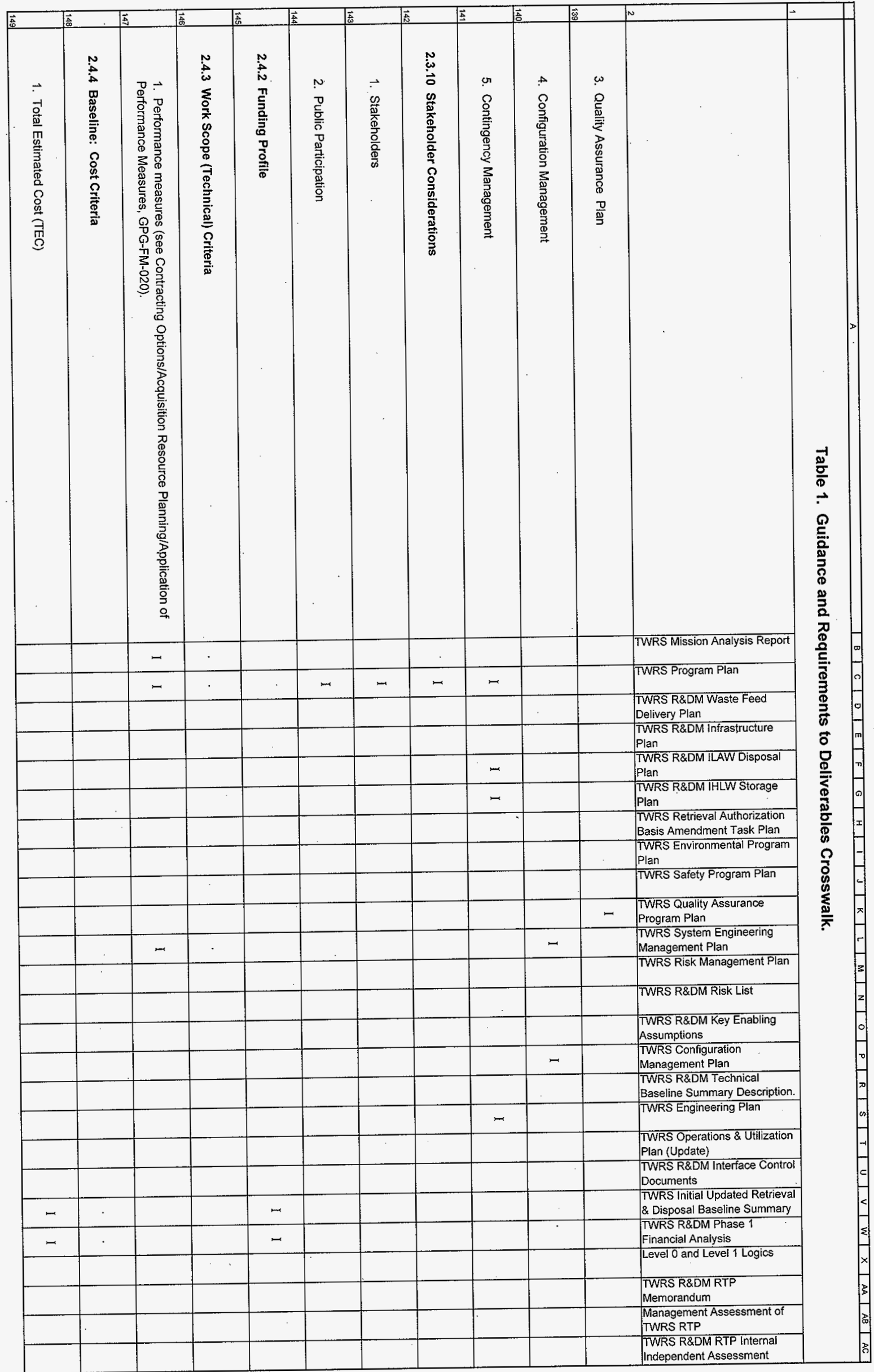




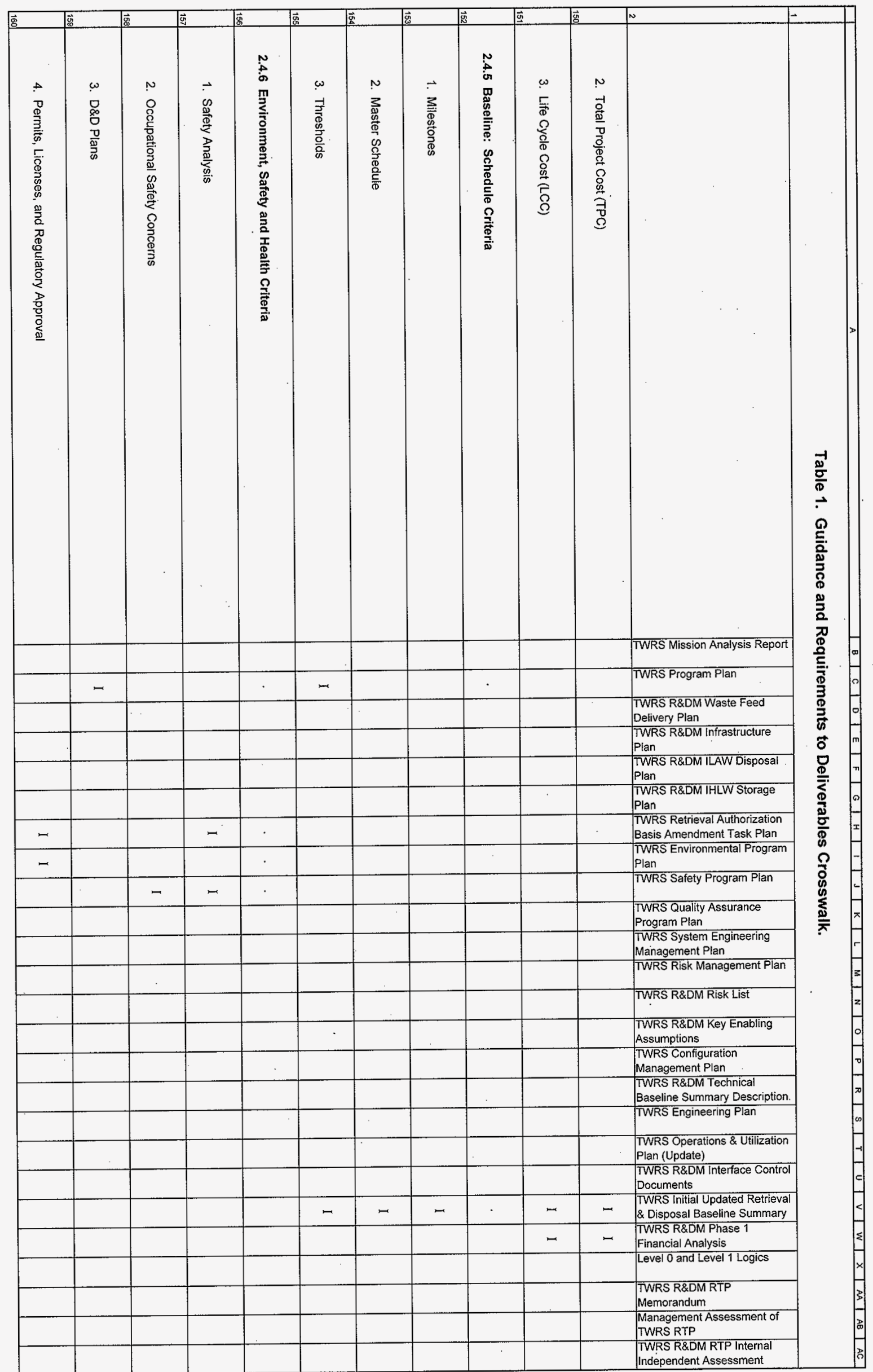




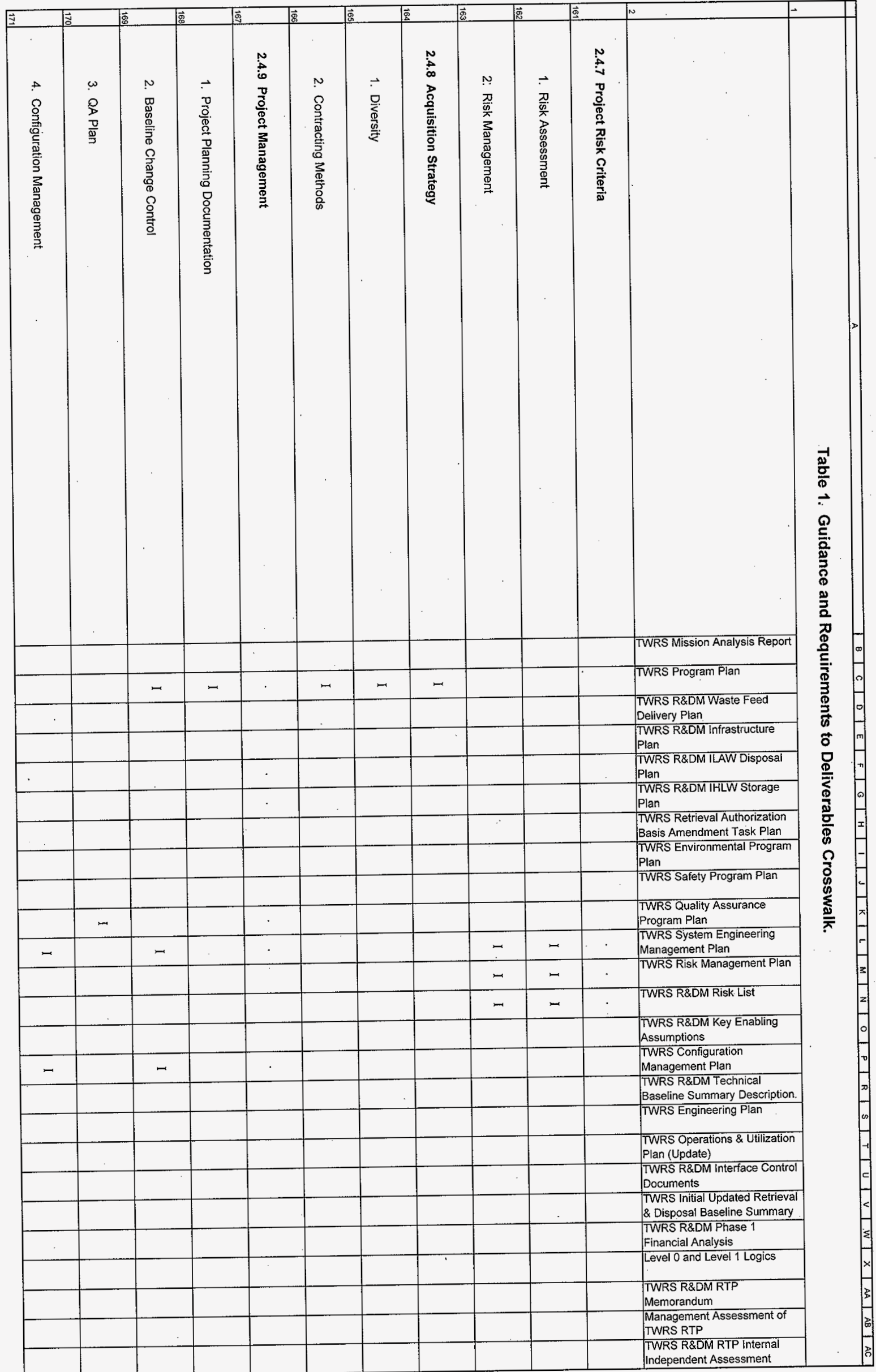




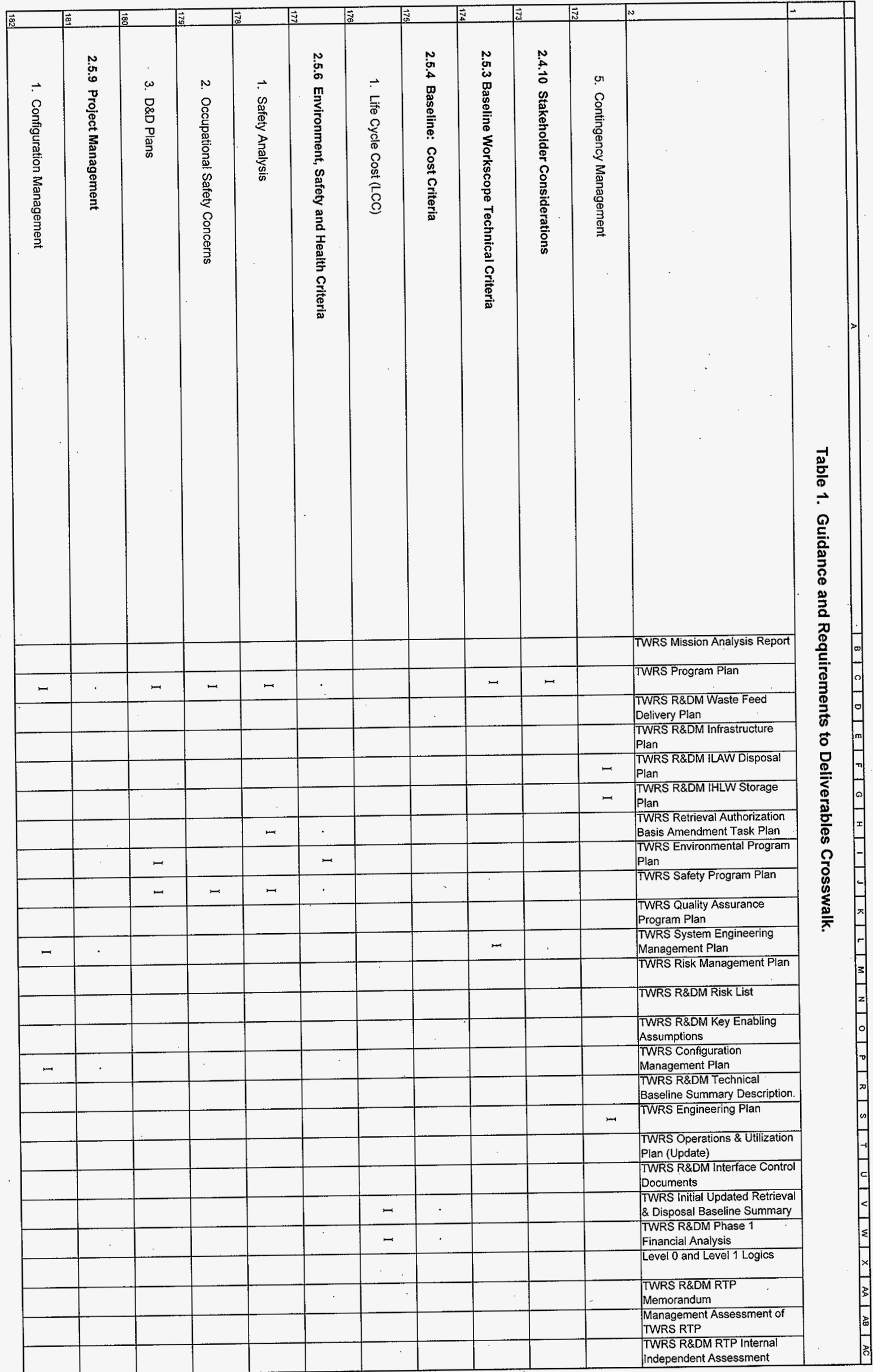





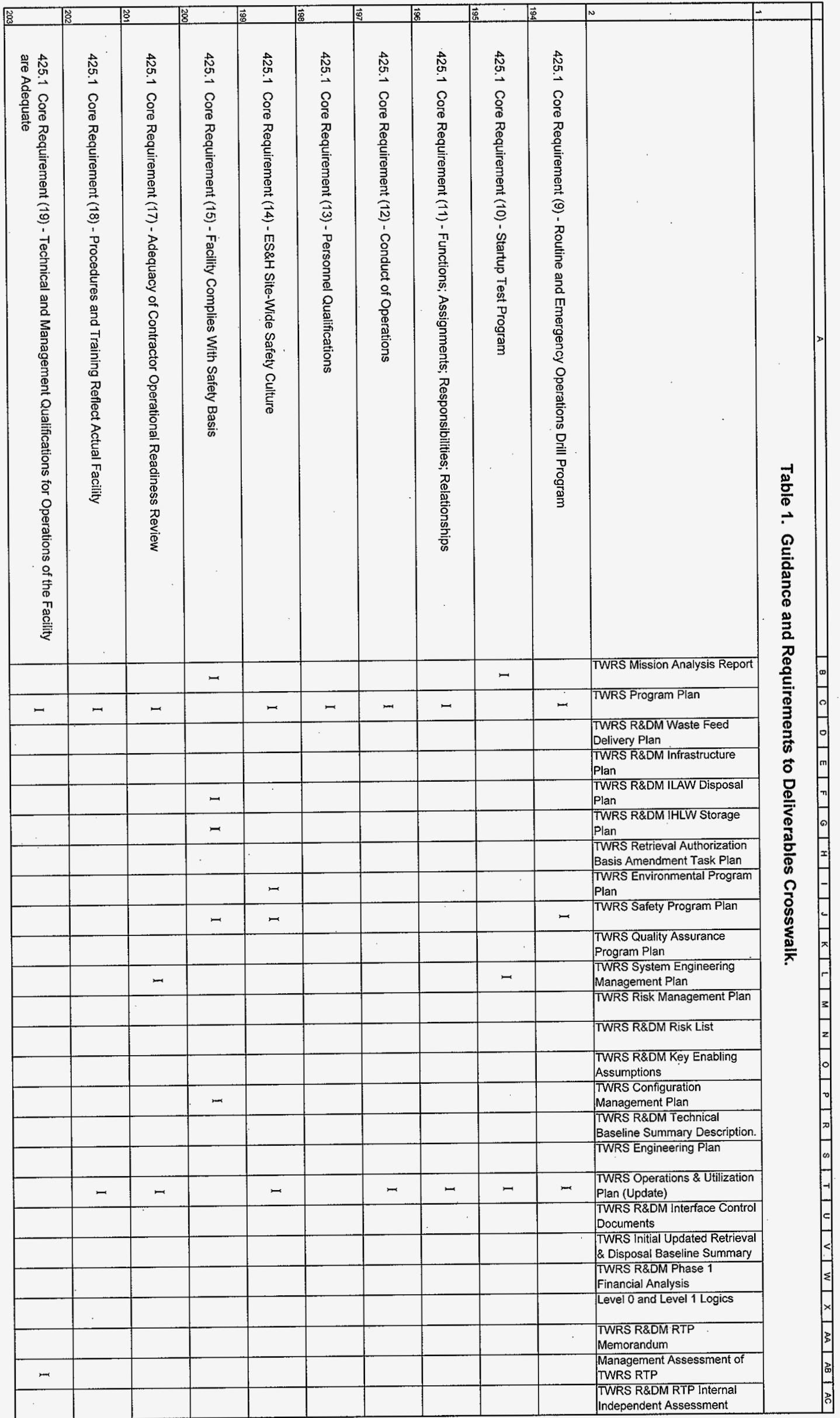




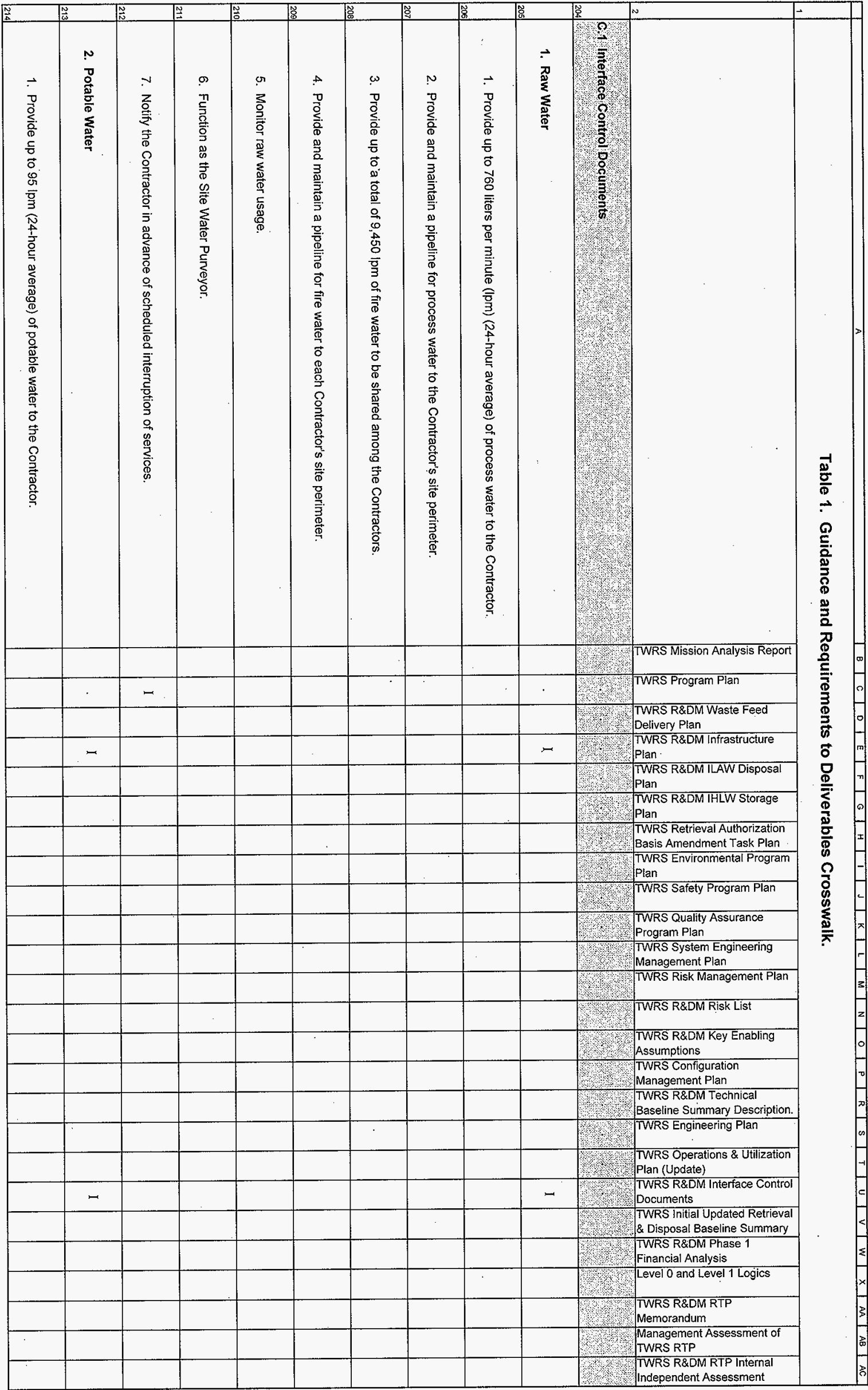




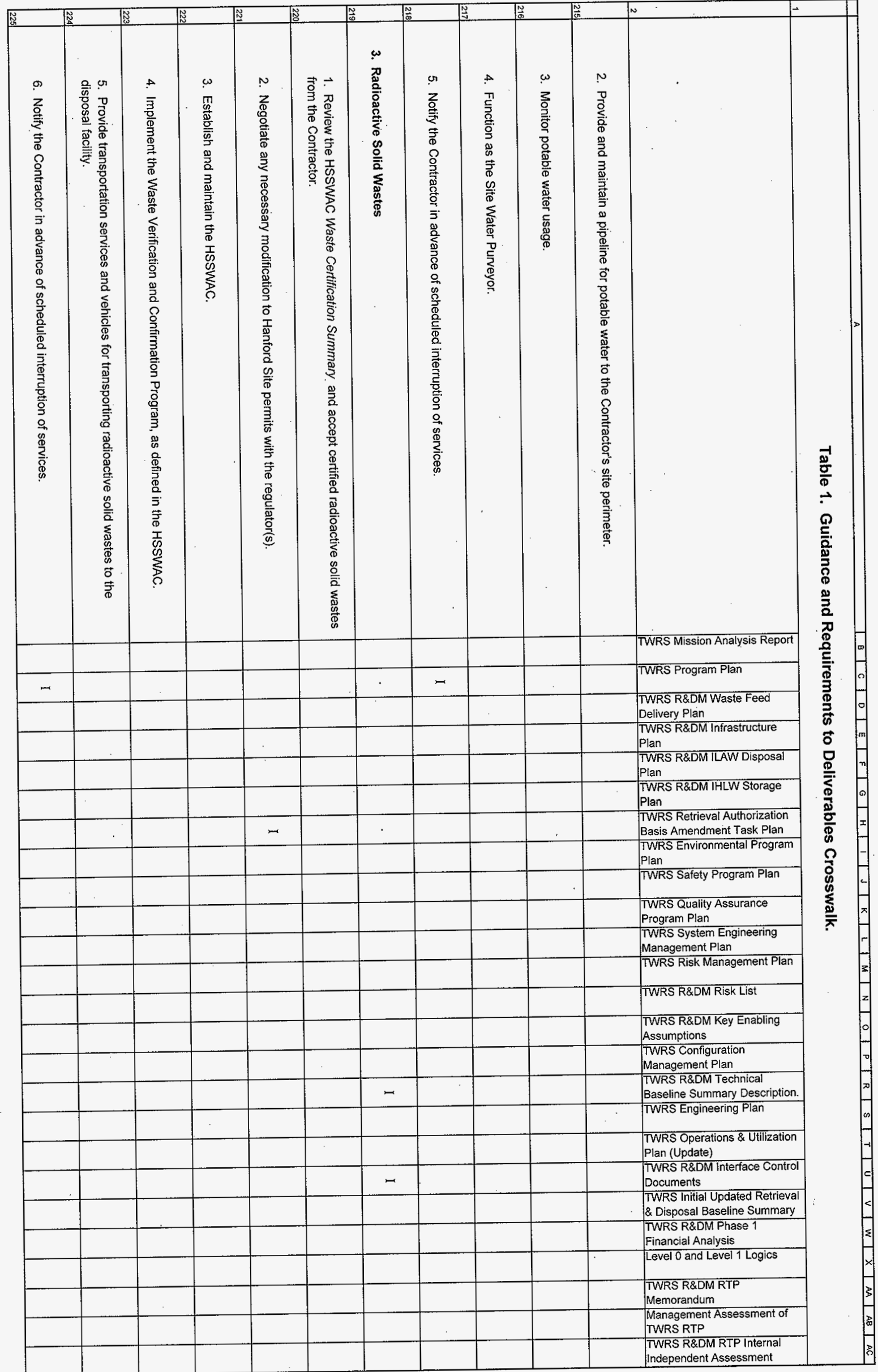




\begin{tabular}{|c|c|c|c|c|c|c|c|c|c|c|c|c|}
\hline & 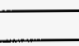 & & 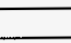 & & 㩊 & 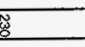 & ב & 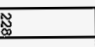 & 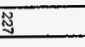 & 工 & N & 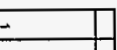 \\
\hline 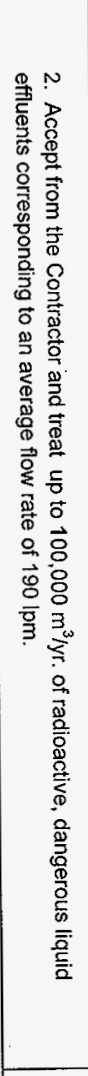 & 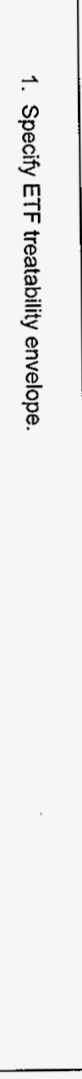 & 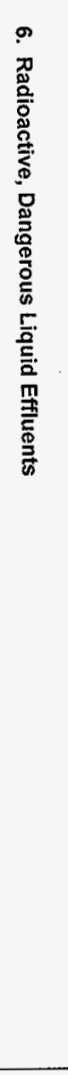 & 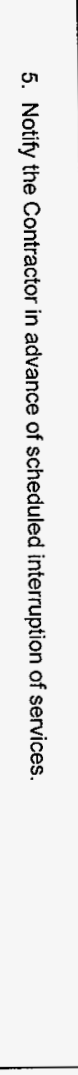 & 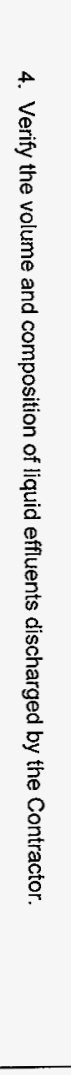 & 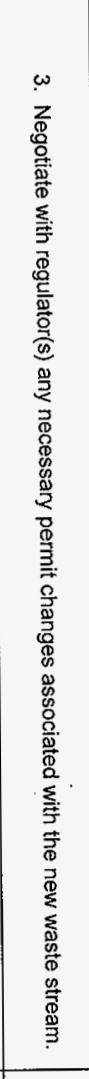 & 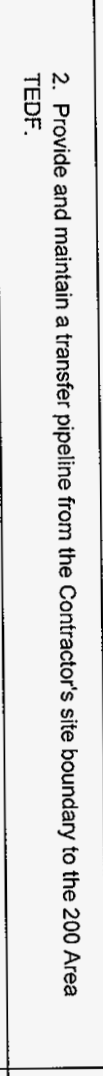 & 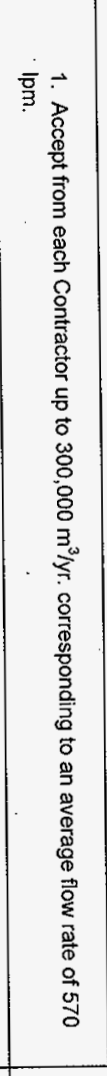 & 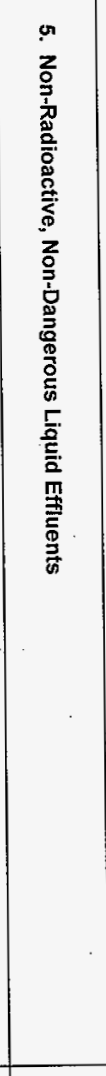 & 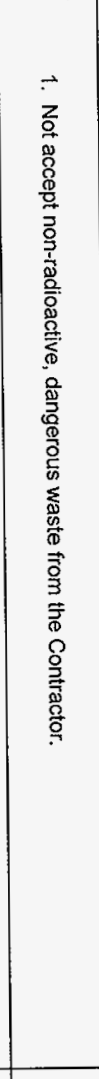 & 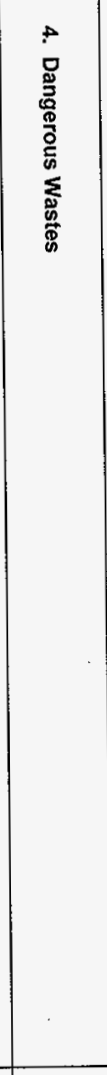 & & 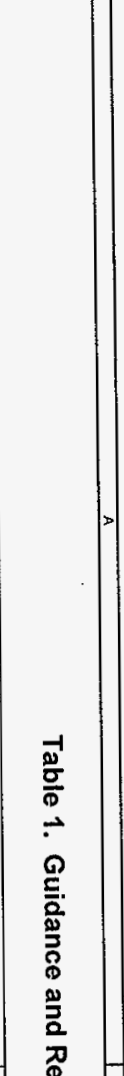 \\
\hline & & & & & & & & & & & TWRS Mission Analysis Report & 总 \\
\hline & & . & - & & & & & . & & & TWRS Program Plan & $\overline{9}$ \\
\hline & & & & & & & & & & & $\begin{array}{l}\text { TWRS R\&DM Waste Feed } \\
\text { Delivery Plan }\end{array}$ & $\stackrel{D}{\stackrel{D}{*}}$ \\
\hline & & & & & & & & & & & $\begin{array}{l}\text { TWRS R\&DM Infrastructure } \\
\text { Plan }\end{array}$ & $\tilde{0}$ \\
\hline & & & & & & & & & & & $\begin{array}{l}\text { TWRS R\&DM ILAW Disposai } \\
\text { Plan }\end{array}$ & $\underset{\Phi}{=}$ \\
\hline & & & & & & & & & & & $\begin{array}{l}\text { TWRS R\&DM IFLW Storage } \\
\text { Plan }\end{array}$ & $\widehat{\underline{0}}$ \\
\hline & & - & & & & & & 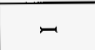 & $m$ & & $\begin{array}{l}\text { TWRS Retrieval Authorization } \\
\text { Basis Amendment Task Plan }\end{array}$ & $\frac{\sigma}{0}$ \\
\hline & & & & & & & & & & & $\begin{array}{l}\text { TWRS Environmental Program } \\
\text { Plan }\end{array}$ & $\stackrel{\infty}{\infty}$ \\
\hline & & & & & & & & & & & TWRS Safety Program Plan & 点 \\
\hline & & & & & & & & & & & $\begin{array}{l}\text { TWRS Quality Assurance } \\
\text { Program Plan }\end{array}$ & 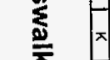 \\
\hline & & & & & & & & & & & $\begin{array}{l}\text { TWRS System Engineering } \\
\text { Management Plan }\end{array}$ & \\
\hline & & & & & & & & & & & ITWRS Risk Management Plan & \\
\hline & & & & & & & & & & & TWRS R\&DM Risk List & \\
\hline & & & & & & & & & & & $\begin{array}{l}\text { TWRS R\&DM Key Enabling } \\
\text { Assumptions }\end{array}$ & \\
\hline & & & & & & & & & & & $\begin{array}{l}\text { TWRS Configuration } \\
\text { Management Plan }\end{array}$ & \\
\hline & & & & & & & & & & & $\begin{array}{l}\text { TWRS R\&DM Technical } \\
\text { Baseline Summary Description. }\end{array}$ & \\
\hline & & & & & & & & & & & TWRS Engineering Plan & \\
\hline & & & & & & & & & & & $\begin{array}{l}\text { TwRS Operations \& Ufilization } \\
\text { Plan (Update) }\end{array}$ & \\
\hline & & - & & & & & & - & - & . & $\begin{array}{l}\text { TW/RS R\&DM Interface Control } \\
\text { Documents }\end{array}$ & \\
\hline & & & & & & & & & & & $\begin{array}{l}\text { TWRS Intial Updated Retrieval } \\
\text { \& Disposal Baseline Summary }\end{array}$ & \\
\hline & & & & & & & & & & & \begin{tabular}{|l|} 
TWRS R\&DM Phase 1 \\
Financial Analysis
\end{tabular} & \\
\hline & & & & & & & & & & & 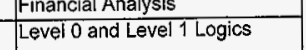 & \\
\hline & & & & & & & & & & & TWRS R\&DM RTP & \\
\hline & & & & & & & & & & & $\begin{array}{l}\text { Memorandum } \\
\text { Manacement Assessment of }\end{array}$ & \\
\hline & & & & & & & & & & & 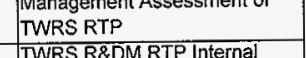 & \\
\hline & & & & & & & & & & & $\begin{array}{l}\text { TWRS R\&DM RTP Internal } \\
\text { independent Assessment }\end{array}$ & \\
\hline
\end{tabular}




\begin{tabular}{|c|c|c|c|c|c|c|c|c|c|c|c|c|}
\hline- & . & & & & - & & 莡 & $=$ & 䈏 & $\bar{\square}$ & F & $\rightarrow$ \\
\hline 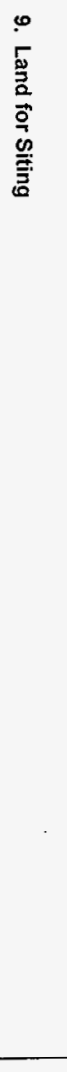 & 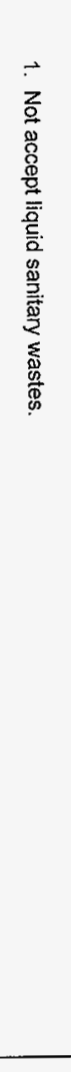 & 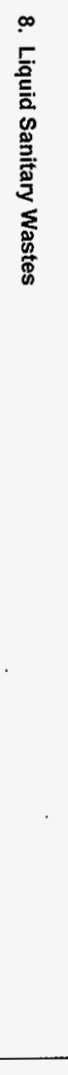 & 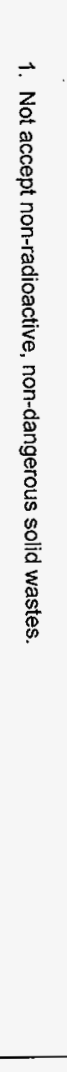 & 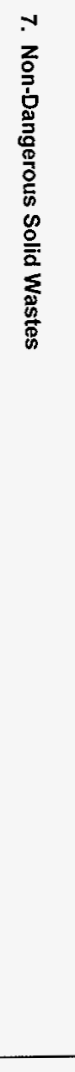 & 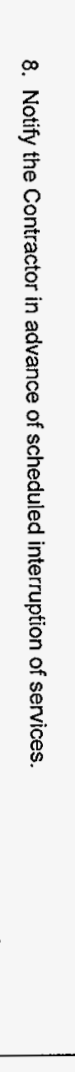 & 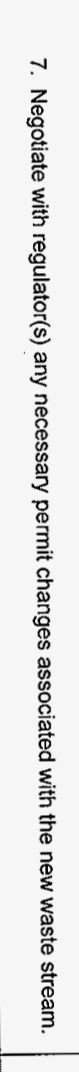 & 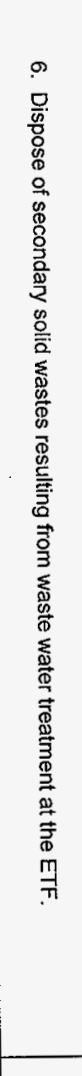 & 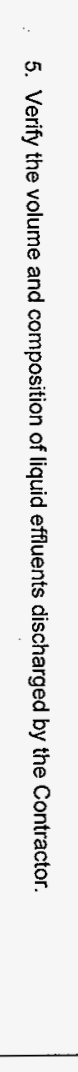 & 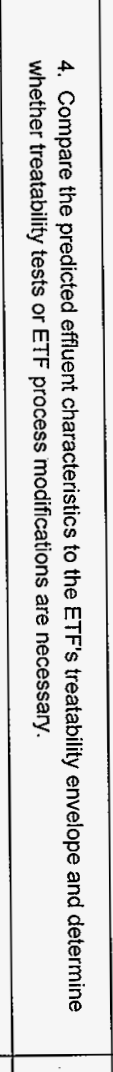 & 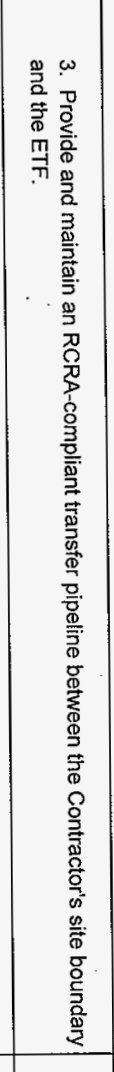 & & 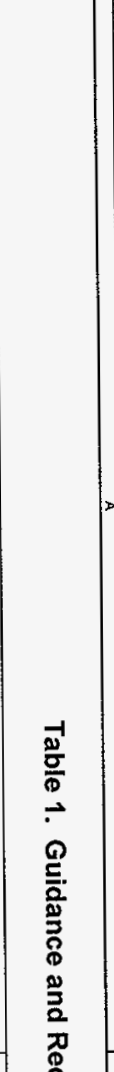 \\
\hline & & & & & & & & & & & \begin{tabular}{|l|} 
TWRS Mission Analysis Report \\
TWRS Program Plan
\end{tabular} & $\stackrel{8}{8}$ \\
\hline & & & & & - & & & & & & \begin{tabular}{|l} 
TWRS Program Plan \\
TWRS R\&DM Waste Feed
\end{tabular} & $\stackrel{\Phi}{\Xi}$ \\
\hline & & & & & & & & & & & $\begin{array}{l}\text { TWRS R\&DM Waste Feed } \\
\text { Delilevry Plan } \\
\text { TWRS R\&DM Infrastructure }\end{array}$ & 市 \\
\hline$m$ & & & & & & & & & & & $\begin{array}{l}\text { WTRS R\&DM Infrastructure } \\
\text { plan }\end{array}$ & it \\
\hline & & & & & & & & & & & $\begin{array}{l}\text { TWRS R\&DM ILAW Disposal } \\
\text { Plan }\end{array}$ & $\stackrel{\text { D }}{=}$ \\
\hline & & & & & & & & & & & $\begin{array}{l}\text { TWRS R\&DM IHLW Storage } \\
\text { Plan }\end{array}$ & 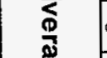 \\
\hline & & & & & & & & & & & $\begin{array}{l}\text { TWRS Retrieval Authorization } \\
\text { Basis Amendment Task Plan } \\
\end{array}$ & $\frac{\sigma}{\phi}$ \\
\hline & & & & & & & & & & & $\begin{array}{l}\text { TWRS Environmental Program } \\
\text { Plan }\end{array}$ & ? \\
\hline & & & & & & & & & & & TWRS Safety Program Plan & 品 \\
\hline & & & & & & & & & & & $\begin{array}{l}\text { TWRS Quality Assurance } \\
\text { Program Plan }\end{array}$ & 王 \\
\hline & & & & & & & & & & & $\begin{array}{l}\text { TWRS System Engineering } \\
\text { Management Plan }\end{array}$ & \\
\hline & & & & & & & & & & & TWRS Risk Management Plan & \\
\hline & & & & & & & & & & & TWRS R\&DM Risk List & \\
\hline & & & & & & & & & & & $\begin{array}{l}\text { TWRS R\&DM Key Enabling } \\
\text { Assumptions }\end{array}$ & \\
\hline & & & & & & & & & & & \begin{tabular}{|l} 
TWRS Configuration \\
Management Plan
\end{tabular} & \\
\hline & $=$ & & - & . & & & & & & & $\begin{array}{l}\text { TWRS RQDM Technical } \\
\text { Baseline Summary Description. }\end{array}$ & \\
\hline & & & & & & & & & & & TWRS Engineering Plan & \\
\hline & & & & & & & & & & & $\begin{array}{l}\text { TWRS Operations \& Utilization } \\
\text { Plan (Update) }\end{array}$ & \\
\hline- & & - & $=$ & & & & & & & & $\begin{array}{l}\text { TWRS R\&DM I Interface Control } \\
\text { Documents }\end{array}$ & \\
\hline & & & & & & & & & & & \begin{tabular}{|l|} 
Documenis \\
TWRS Initial Updated Retrieval
\end{tabular} & \\
\hline & & & & & & & & & & & \begin{tabular}{|l|} 
\& Disposal Baseline Summary \\
TWRS R\&DM Phase 1
\end{tabular} & \\
\hline & & & & & & & & & & & \begin{tabular}{|l} 
Financial Analysis \\
Level 0 and Level 1 Logics
\end{tabular} & \\
\hline & & & & & & & & & & & TWRS R\&DM RTP & \\
\hline & & & & & & & & & & & $\begin{array}{l}\text { TWR RRDM RTP } \\
\text { Memorandum }\end{array}$ & \\
\hline & & & & & & & & & & & 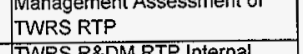 & \\
\hline & & & & & & & & & & & $\begin{array}{l}\text { TWR R RDM RIP Internal } \\
\text { independent Assessment }\end{array}$ & \\
\hline
\end{tabular}




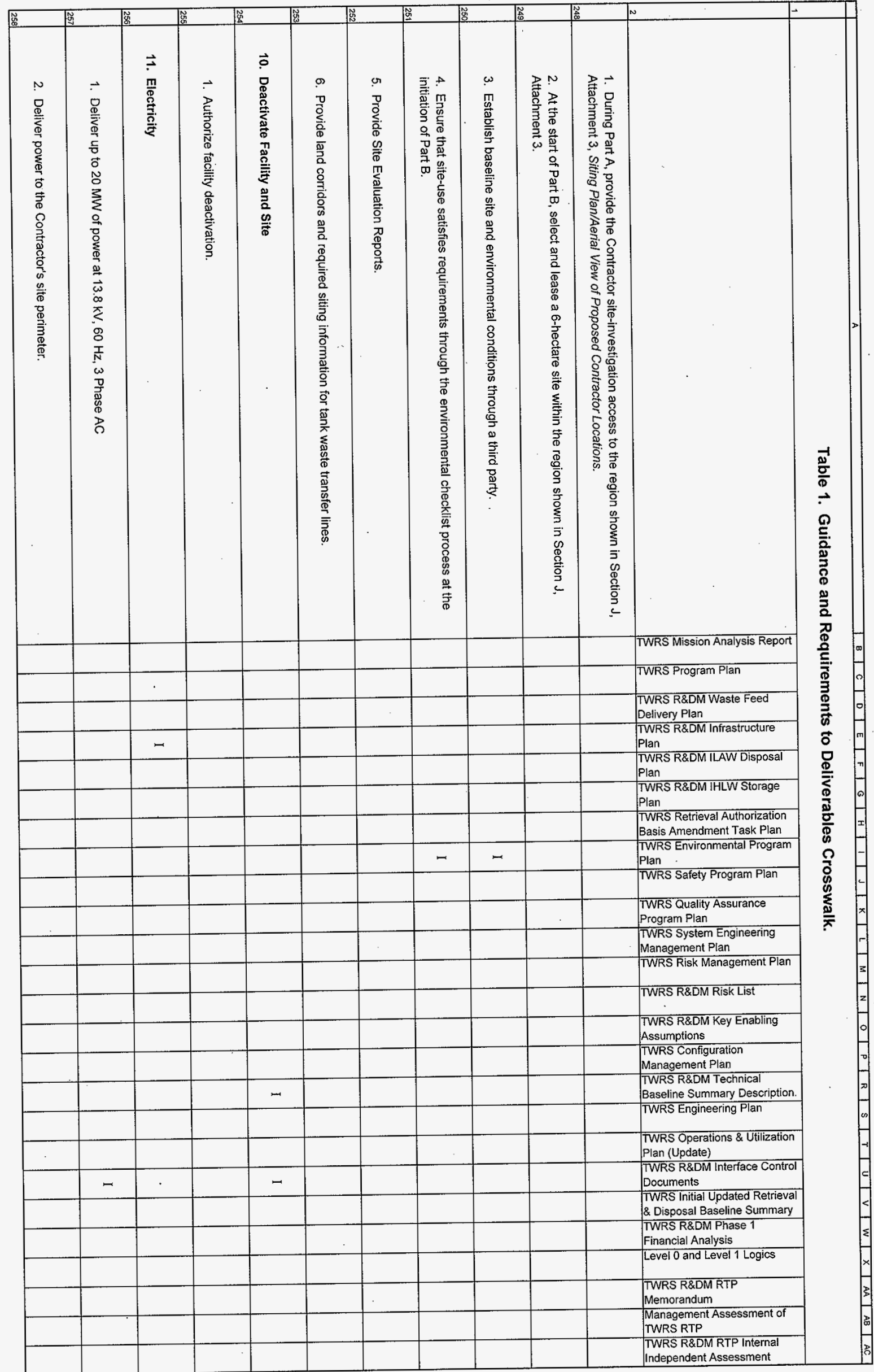




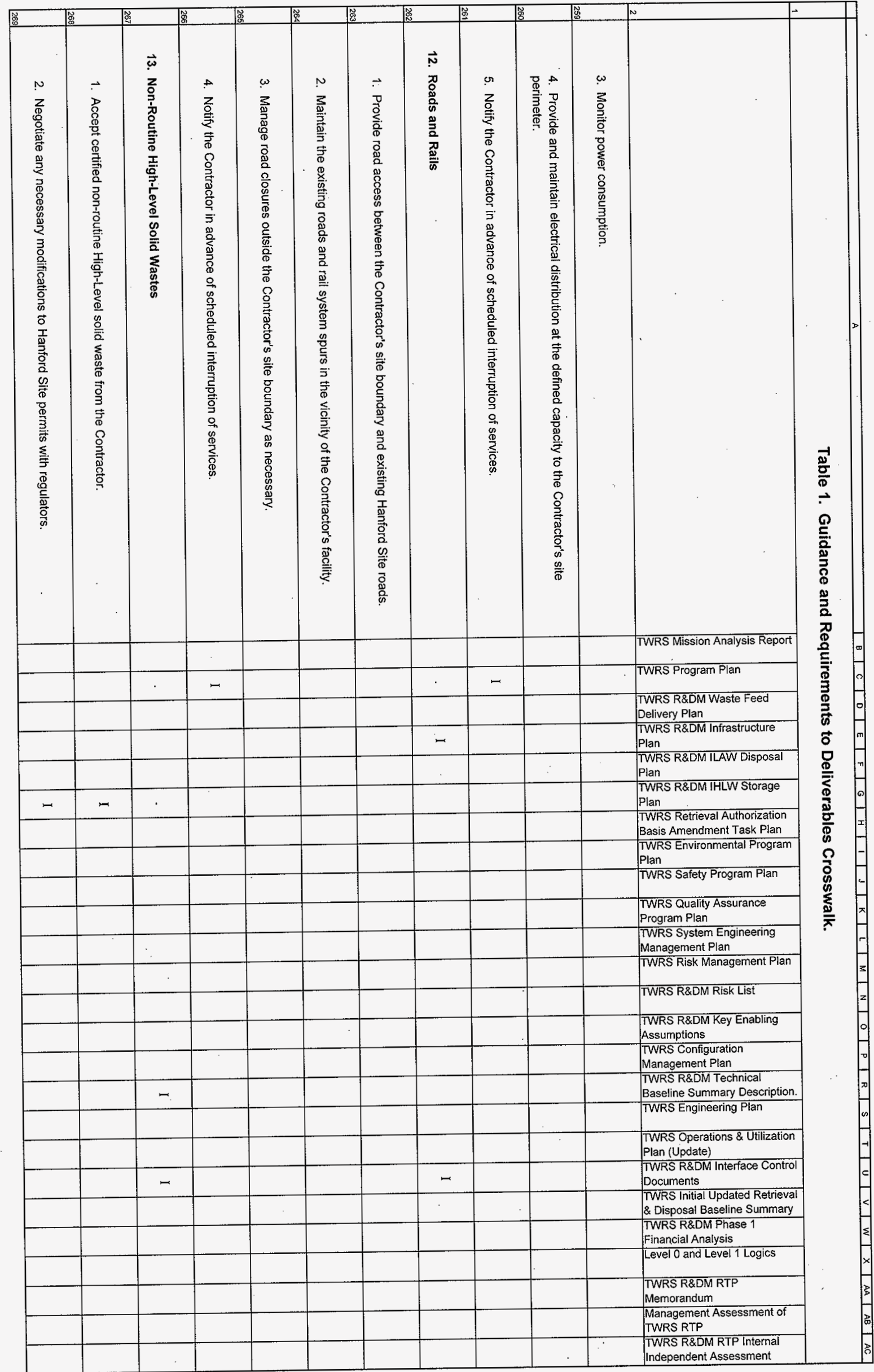




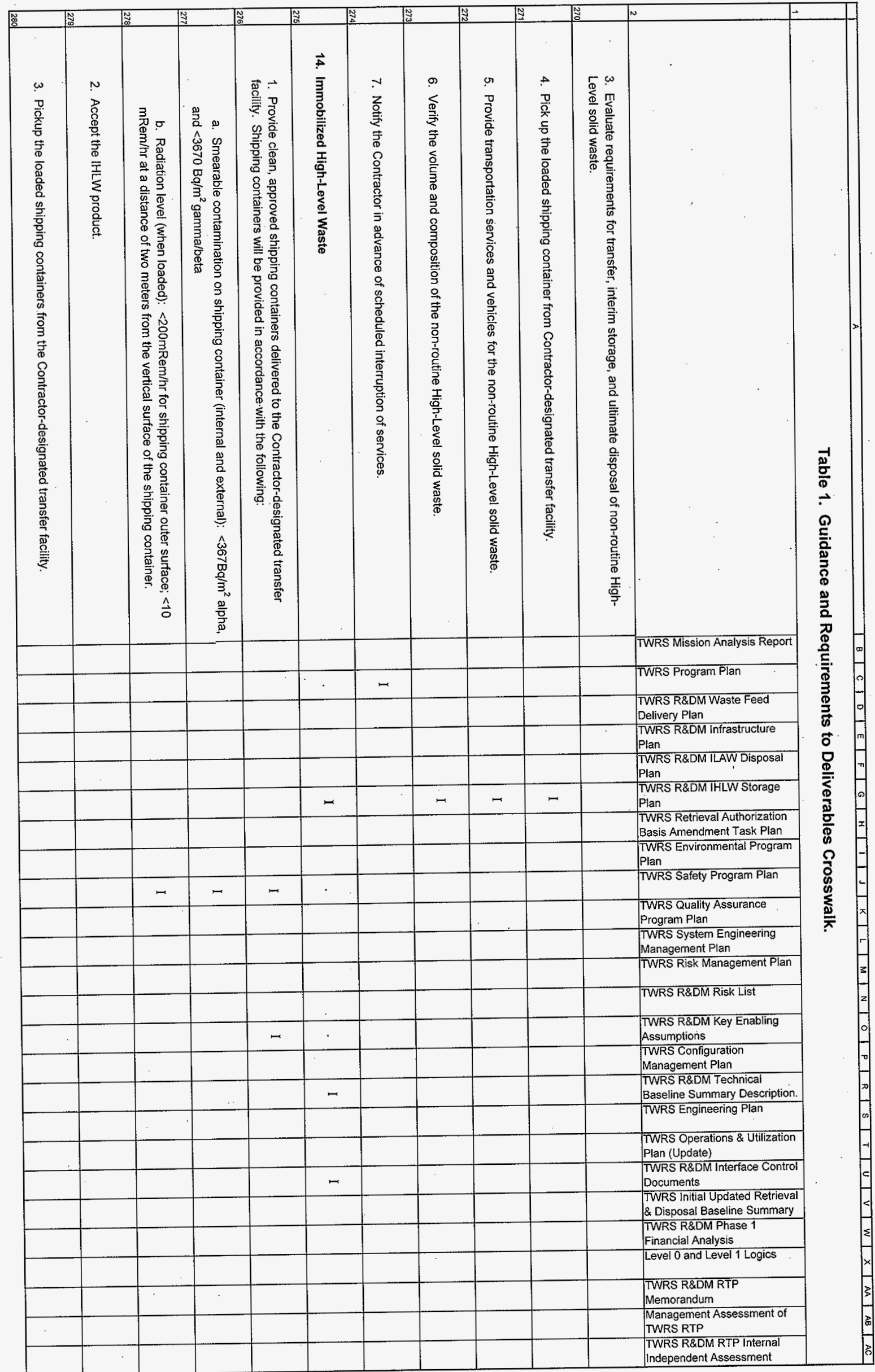




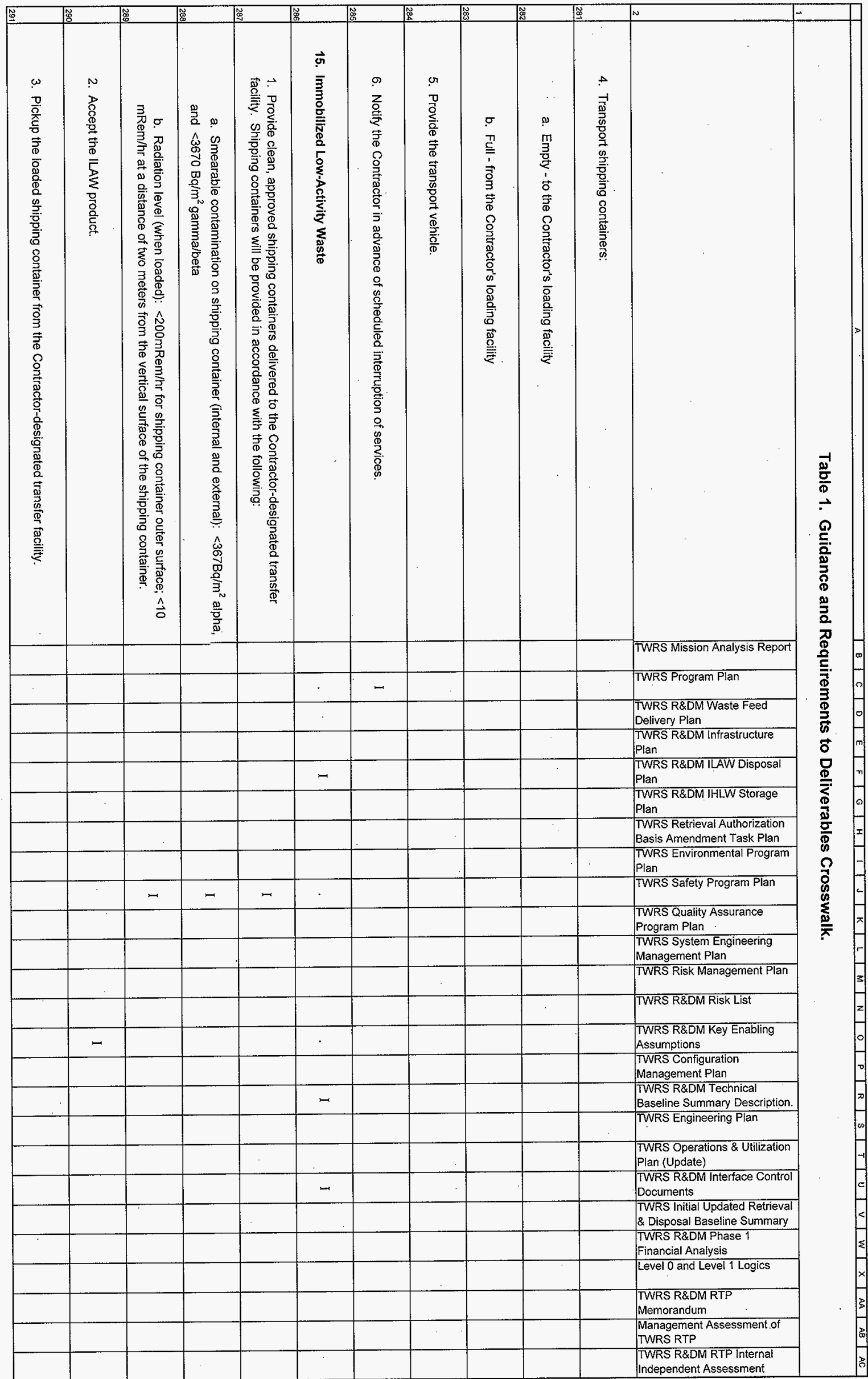




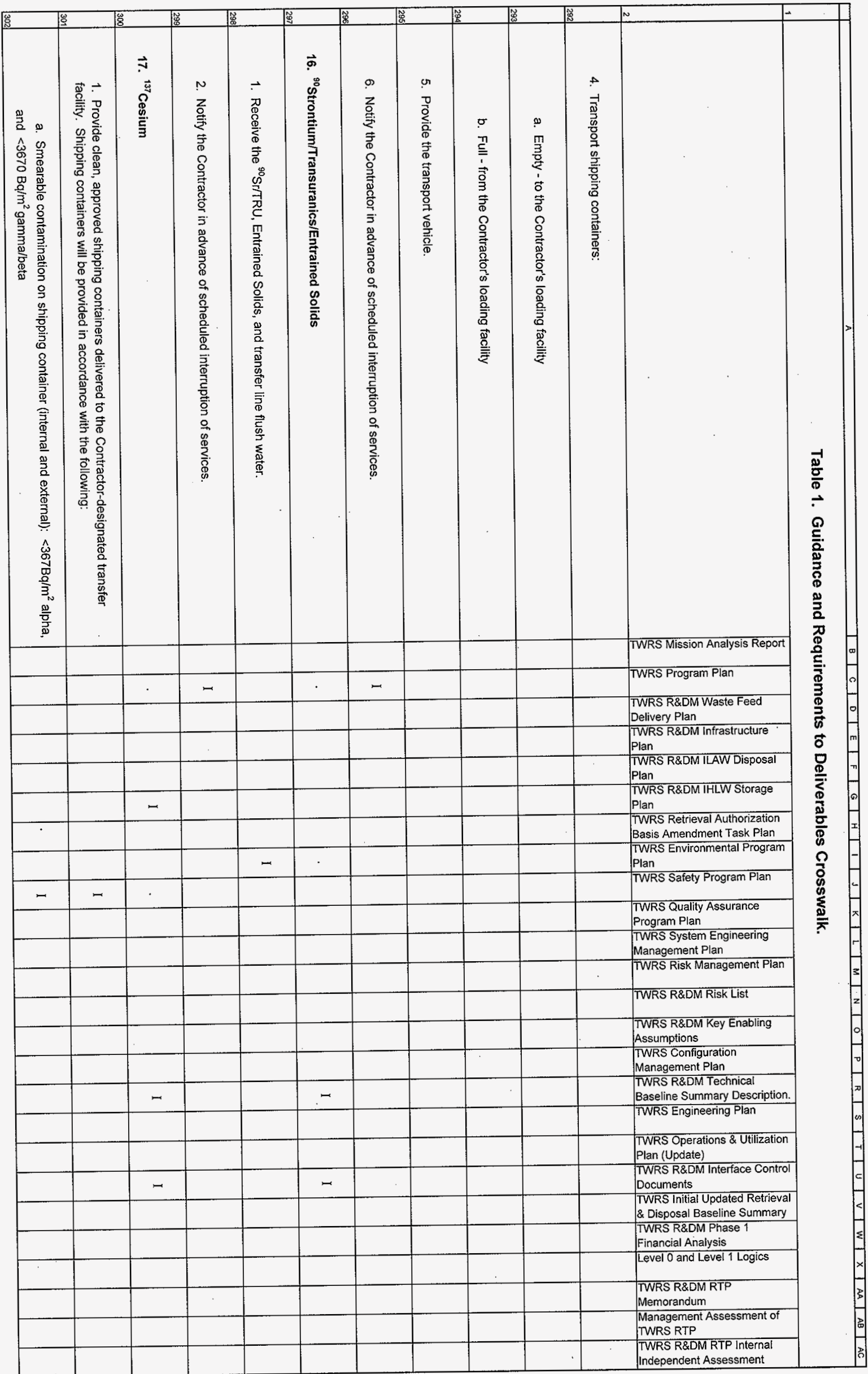




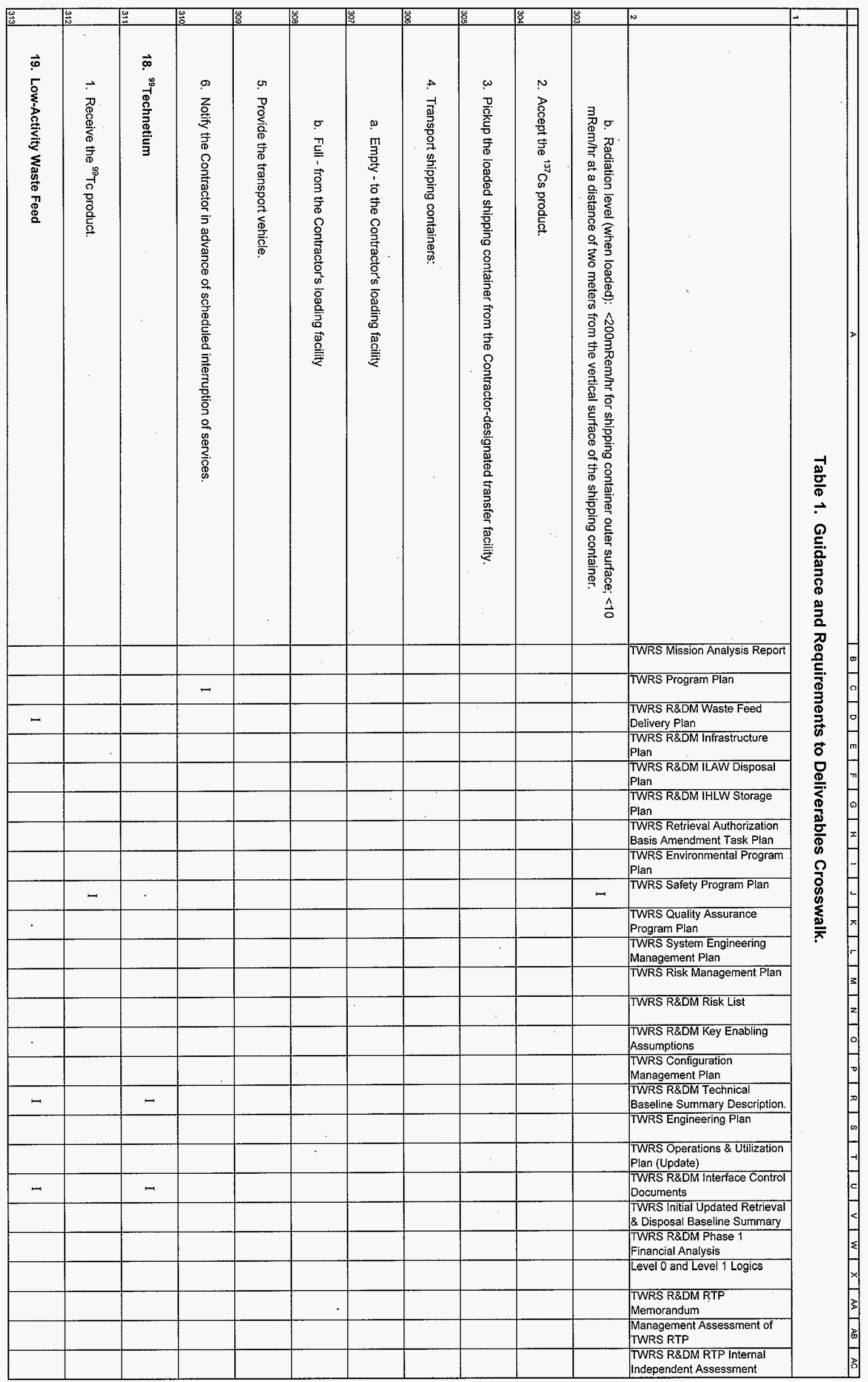




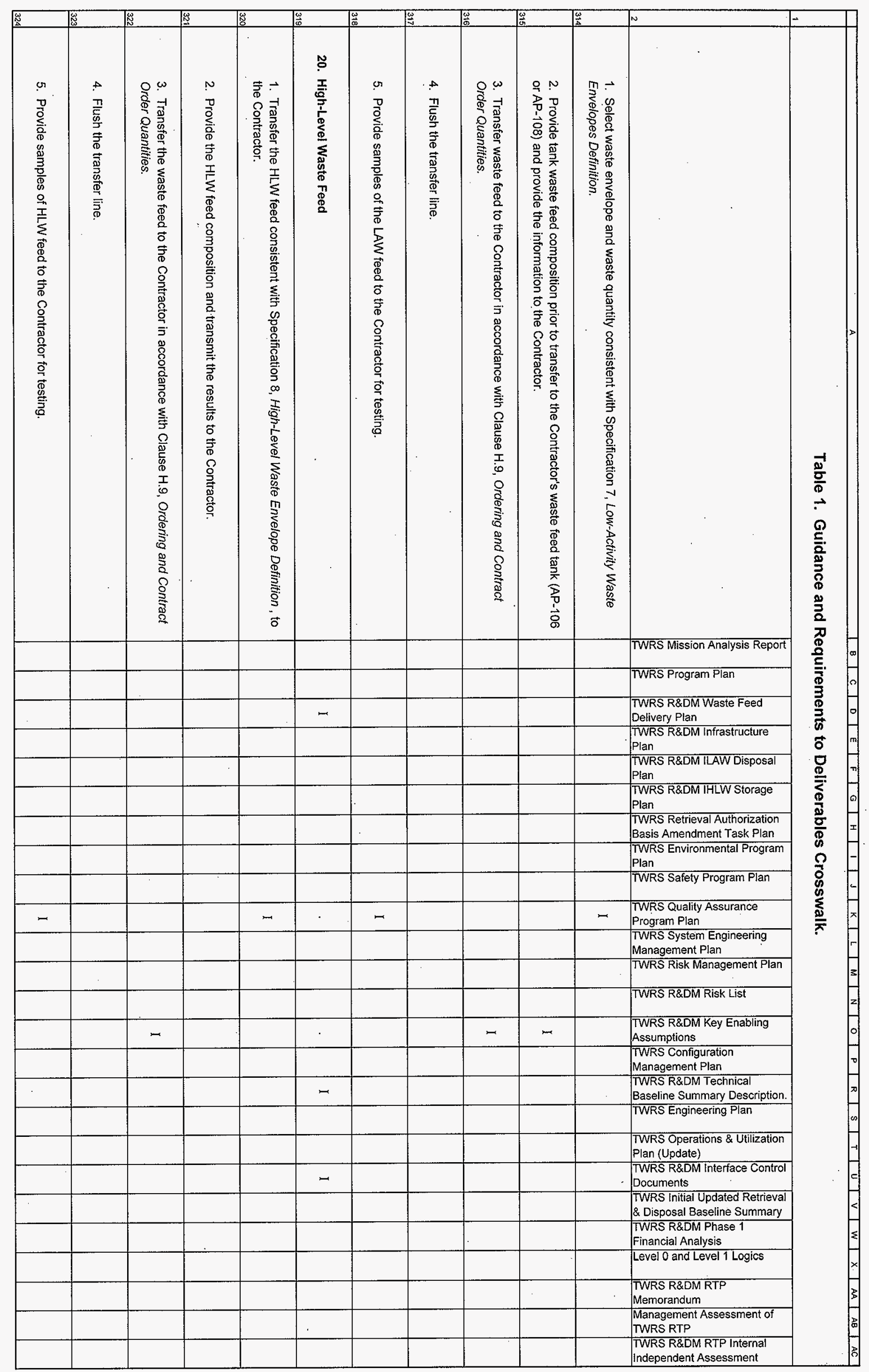




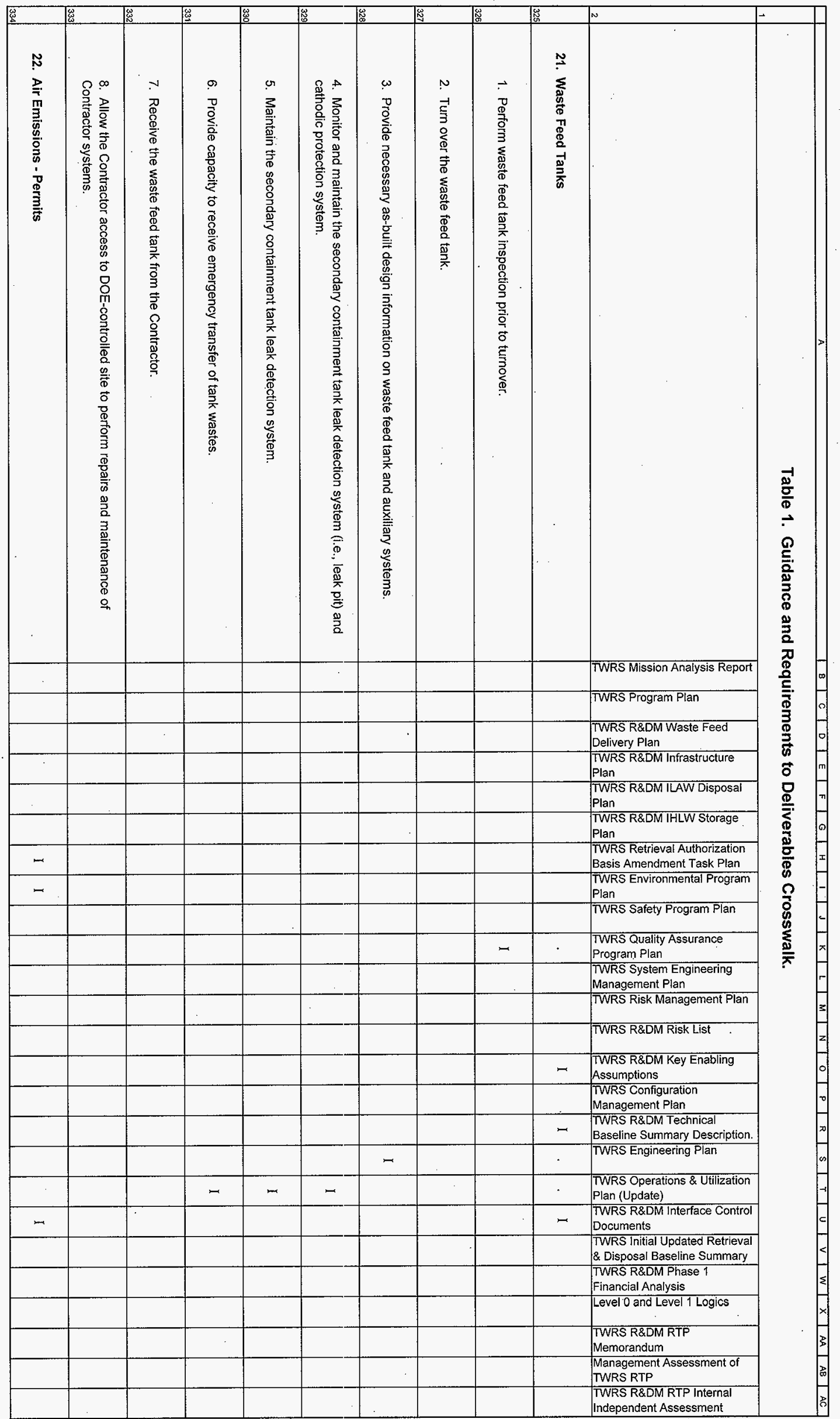




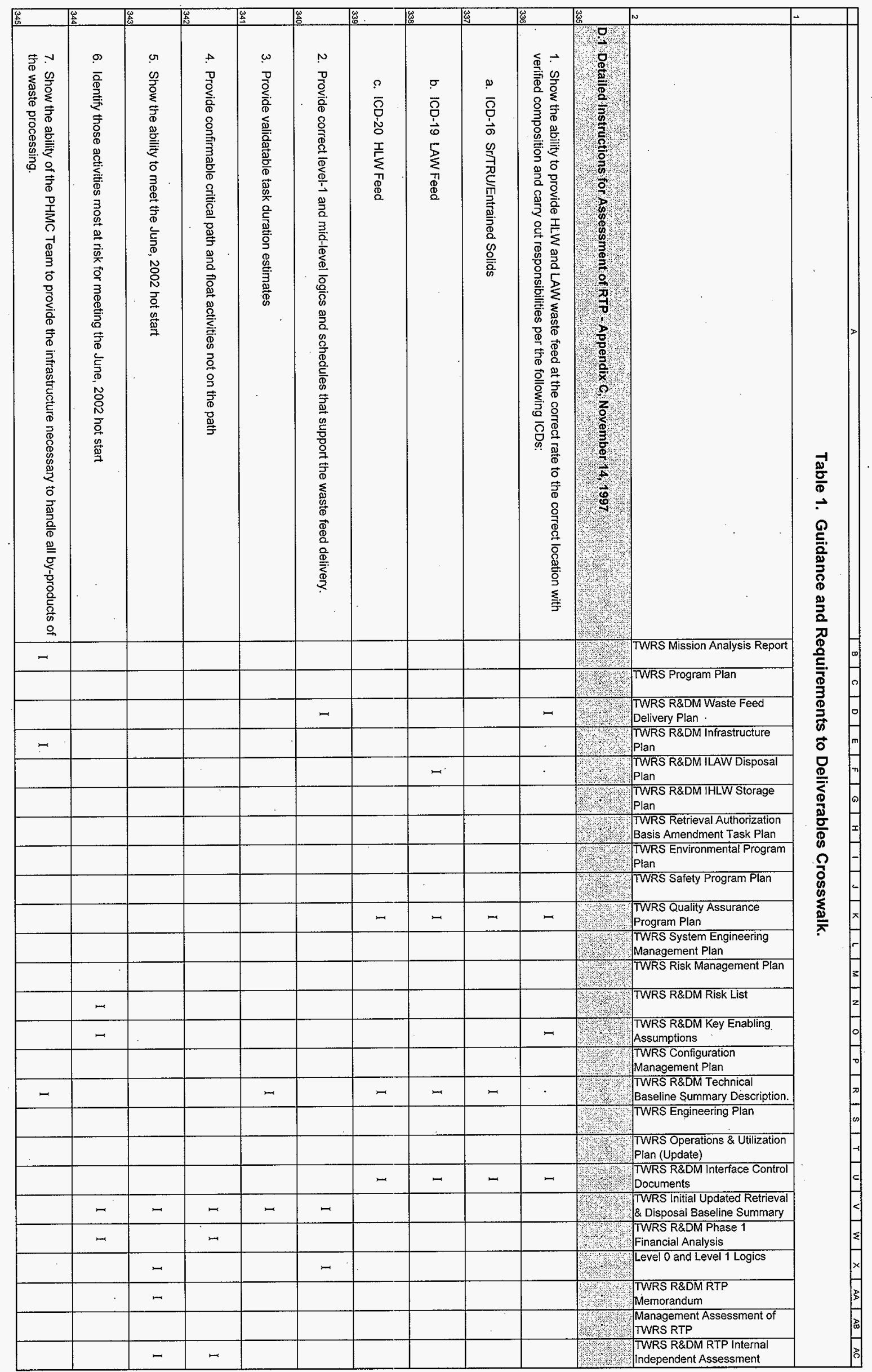




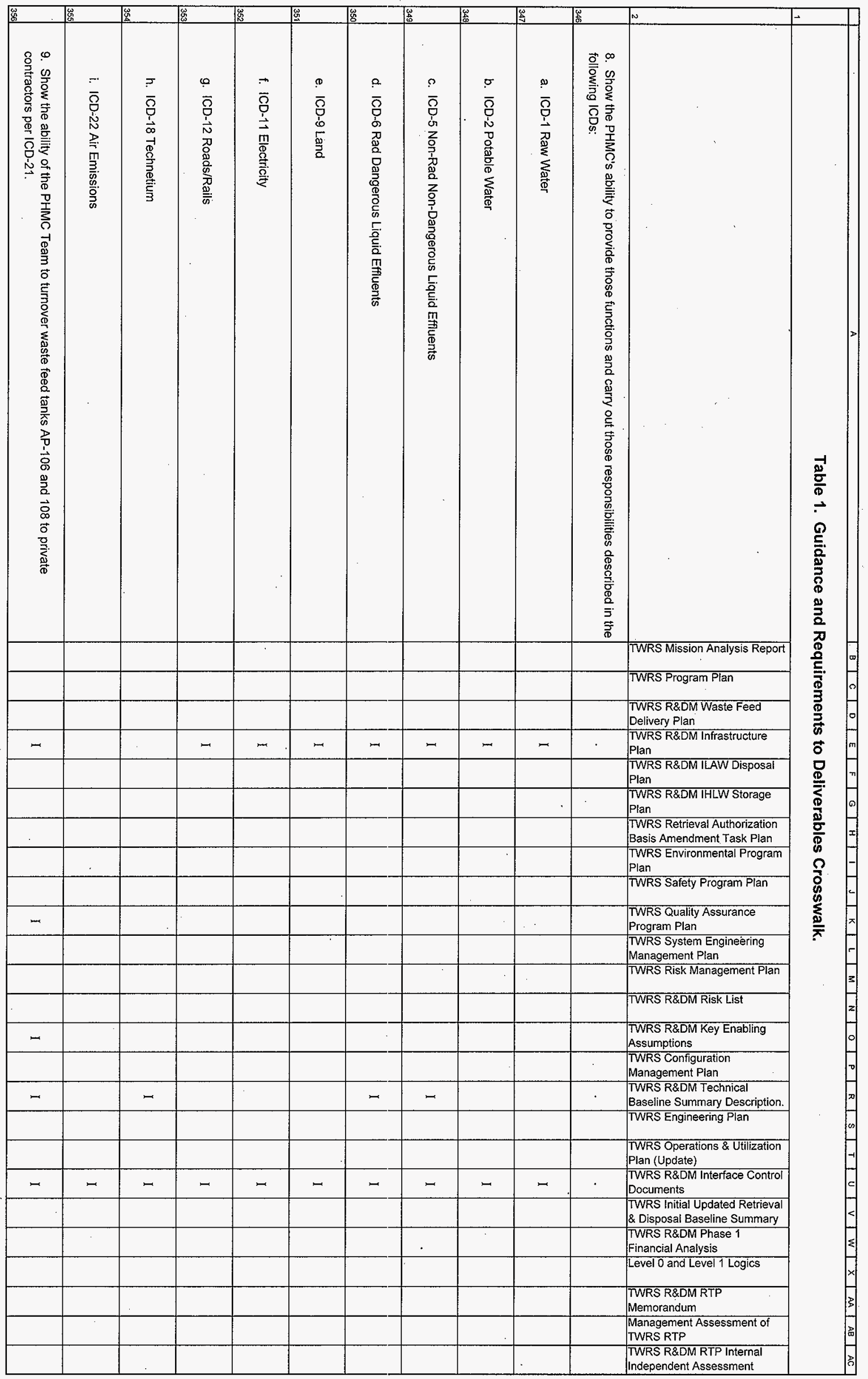




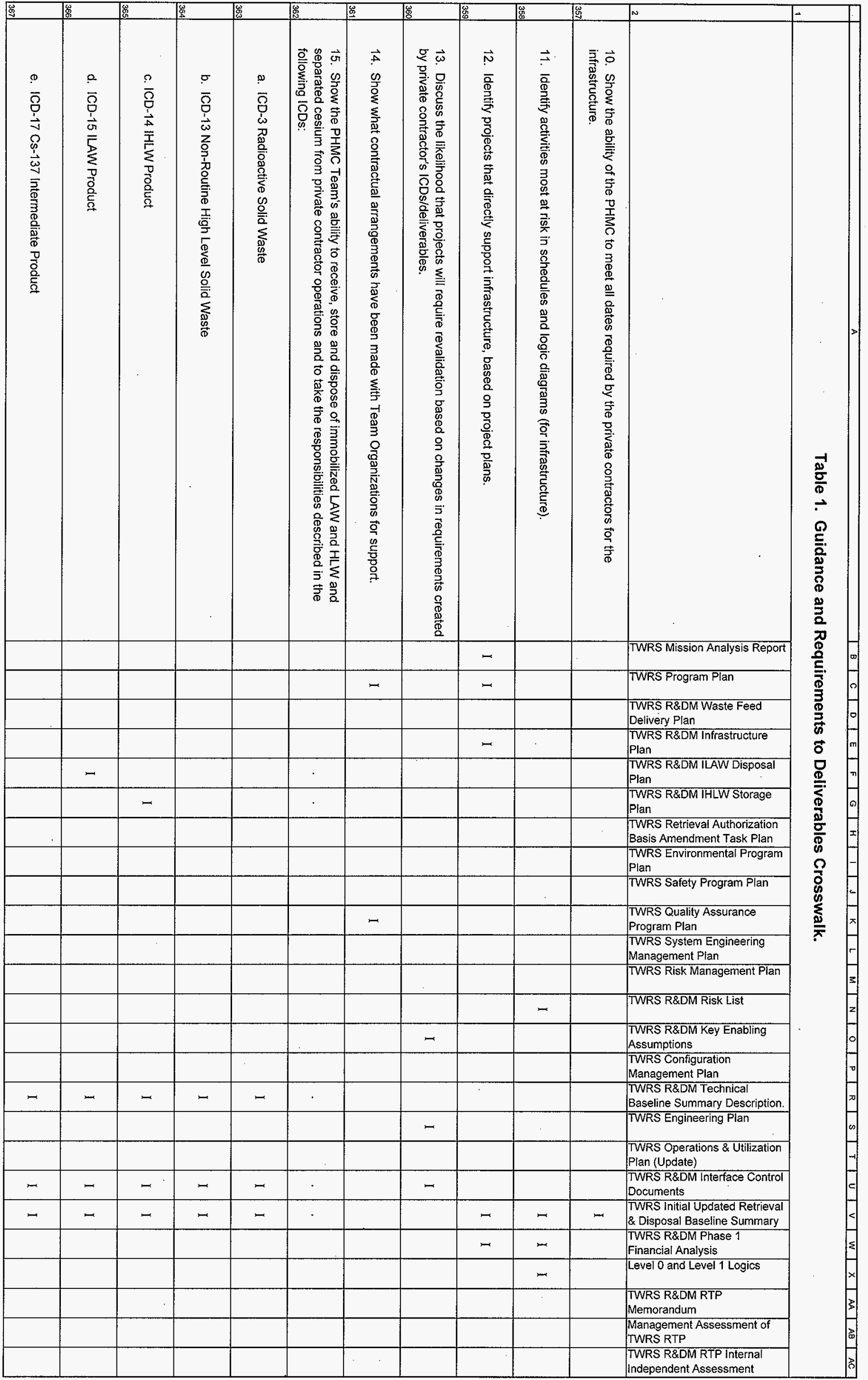




\begin{tabular}{|c|c|c|c|c|c|c|c|c|c|c|c|c|}
\hline & 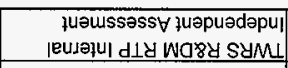 & & & & & 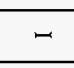 & & & & & & \\
\hline & 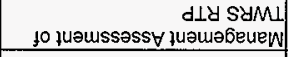 & & & & & - & & & & & & \\
\hline & $\begin{array}{r}\text { unpuedowar } \\
\text { dLy wogy SYMM }\end{array}$ & & & & & $\neg$ & & & & & & \\
\hline & 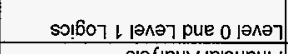 & & $m$ & & & & & & & & & \\
\hline & 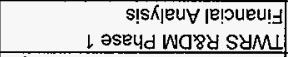 & - & $\sim$ & & & & & & & & & \\
\hline & 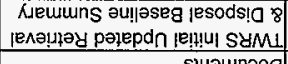 & - & 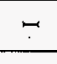 & & & & & & & & & \\
\hline & 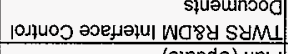 & & & & & & & & & & & \\
\hline & 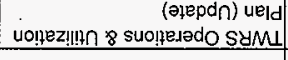 & & & & & & & & & & & \\
\hline & UEld Gu!̣әəulbü SYML & & & & & & & 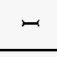 & & & & \\
\hline & 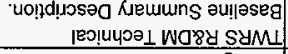 & - & & & & & & & & & & \\
\hline & \begin{tabular}{|r|} 
ueld fuawabeuew \\
uonemblyuos symi \\
\end{tabular} & & & & & & & & & & & \\
\hline & 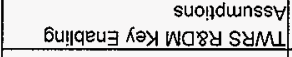 & & & & & - & 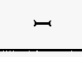 & & & & & \\
\hline & 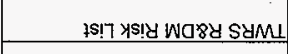 & & - & & & $\sim$ & & - & & & & \\
\hline & 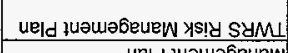 & & & & - & & & & & & & \\
\hline & 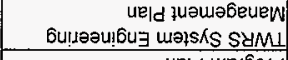 & & & & & . & & & & & & \\
\hline$\overline{\bar{\pi}}$ & 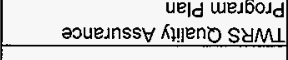 & & & & & & & & $=$ & $=$ & $z$ & \\
\hline : & Ueld welsoodd Riajes SYML & & & & & & - & & & & & \\
\hline$\bigcup_{0}^{\circ}$ & 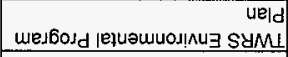 & & & & & & & & & & & \\
\hline$\frac{\frac{\mathrm{\sigma}}{\sigma}}{\frac{\pi}{\pi}}$ & 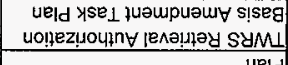 & & & & & & & & & & & \\
\hline 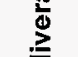 & 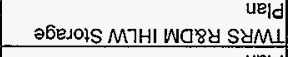 & - & & - & & & & & & & - & \\
\hline$\overline{\bar{\Phi}}$ & |esods!o MV7I wary suld & - & & - & & & & & & & - & \\
\hline$\stackrel{2}{3}$. & 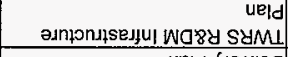 & & & & & & & & & $=$ & & \\
\hline 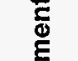 & 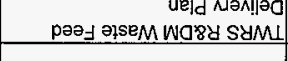 & & & & & & & & - & & & \\
\hline$\stackrel{\overline{0}}{=}$ & 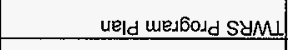 & & & & - & & & & & & & - \\
\hline$\overline{\mathscr{g}}$ & 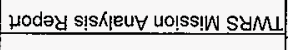 & & & & & & & & & & & \\
\hline 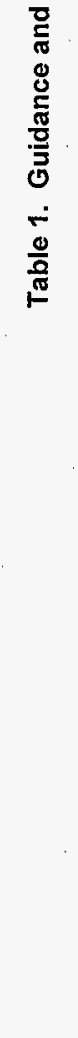 & & 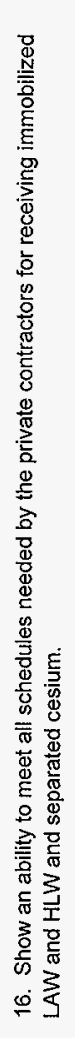 & 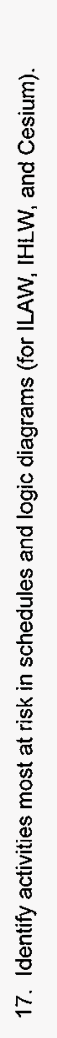 & 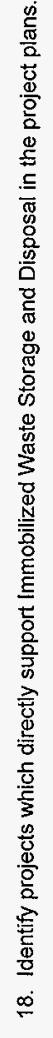 & 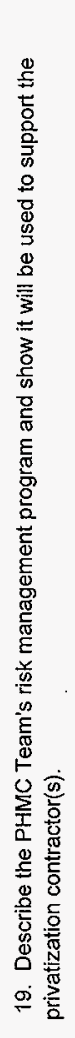 & 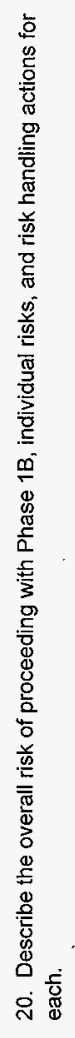 & 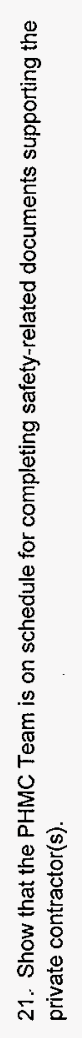 & 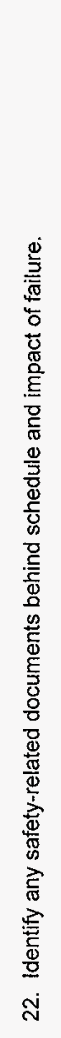 & 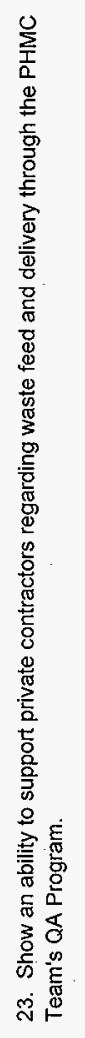 & 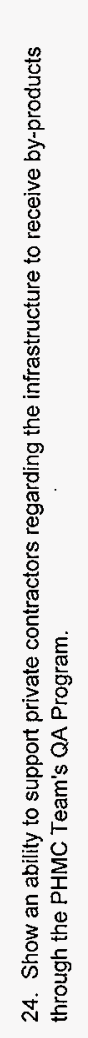 & 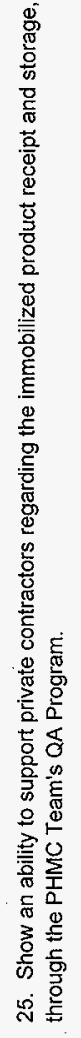 & 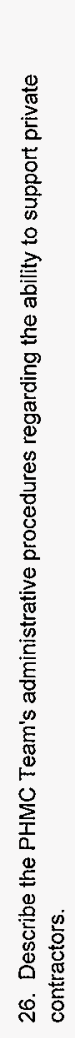 \\
\hline & & & & & & & & & & & & \\
\hline
\end{tabular}




\begin{tabular}{|c|c|c|c|c|c|c|c|c|c|c|c|c|}
\hline & & & & & 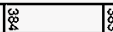 & & & & & & $j^{N}$ & \\
\hline 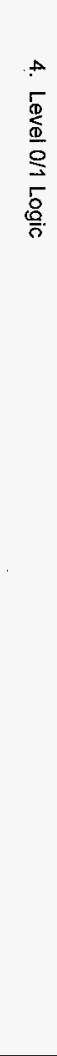 & 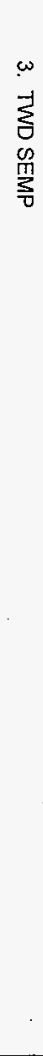 & 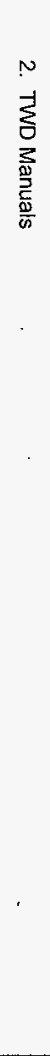 & 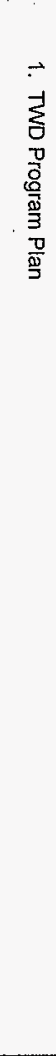 & 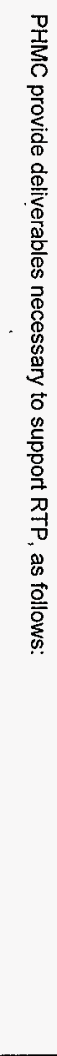 & 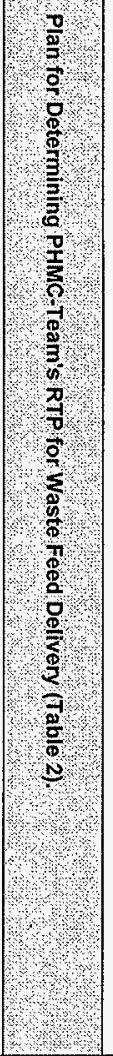 & 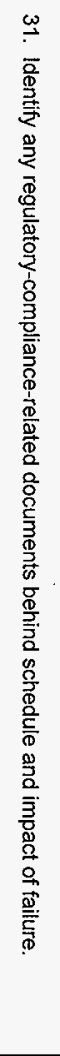 & 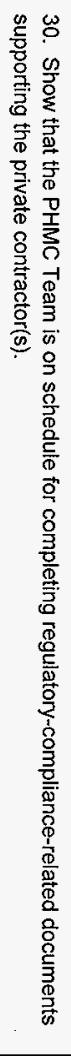 & 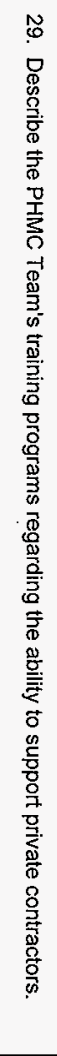 & 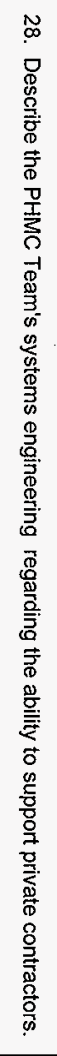 & 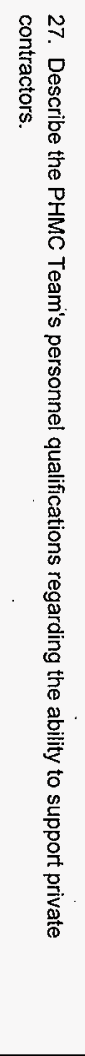 & & 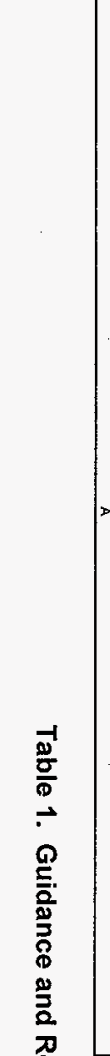 \\
\hline & & & 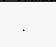 & 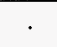 & 13 & & & & & & TWRS Mission Analysis Report & 兽 \\
\hline & & - & - & 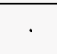 & 1. & & & 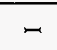 & & $r$ & TWRS Program Plan & 票 \\
\hline & & & & . & -4 & & & & & & $\begin{array}{l}\text { TWRS R\&DM Waste Feed } \\
\text { Delivery Plan }\end{array}$ & $\stackrel{0}{2}$ \\
\hline & & & & & 20 & & & & & & $\begin{array}{l}\text { TWRS REDM Infrastructure } \\
\text { Plan }\end{array}$ & $\frac{\infty}{0}$ \\
\hline & & & & & 30 & & & & & & $\begin{array}{l}\text { TWRS R\&DM ILAW Disposal } \\
\text { Plan }\end{array}$ & $\underset{\Phi}{\underline{Q}}$ \\
\hline & & & & & 1 & & & & & & $\begin{array}{l}\text { TWRS R\&DM IHLLW Storage } \\
\text { Plan }\end{array}$ & $\overline{\bar{\Phi}}$ \\
\hline & & & & . & 3 & - & - & & & & $\begin{array}{l}\text { TWRS Retrieval Authorization } \\
\text { Basis Amendment Task Plan }\end{array}$ & $\frac{5}{8}$ \\
\hline & & & & . & 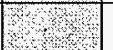 & & $m$ & & & & $\begin{array}{l}\text { TWRS Environmental Program } \\
\text { Plan }\end{array}$ & $\begin{array}{l}0 \\
0 \\
0\end{array}$ \\
\hline & & & & 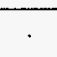 & \% & & - & & & & TWRS Safety Program Plan & 惫 \\
\hline & & & & & 2 & & & & & & $\begin{array}{l}\text { TWRS Quality Assurance } \\
\text { Program Plan }\end{array}$ & है \\
\hline & - & & & . & 28 & & & & - & & $\begin{array}{l}\text { TWRS System Engineering } \\
\text { Management Plan }\end{array}$ & \\
\hline & & & & . & 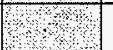 & & & & & & TWRS Risk Management Plan & \\
\hline & & & & & & - & & & & & IWRS R\&DM Risk List & \\
\hline & & & & & & & - & & & & $\begin{array}{l}\text { TWRS R\&DM Key Enabling } \\
\text { Assumptions }\end{array}$ & \\
\hline & & & & & Pon & & & & & & $\begin{array}{l}\text { TWRS Configuration } \\
\text { Management Plan }\end{array}$ & \\
\hline & & & & & & & & & & & $\begin{array}{l}\text { TWRS R\&DM Technical } \\
\text { Baseline Summary Description. }\end{array}$ & \\
\hline & & & & & & & & & & & TWRS Engineering Plan & \\
\hline & & & & & & & & & & & $\begin{array}{l}\text { TWRS Operations \& Utilization } \\
\text { Plan (Update) }\end{array}$ & \\
\hline & & & & & $1 \%$ & & & & & & $\begin{array}{l}\text { FWRS R\&DM Interface Control } \\
\text { Documents }\end{array}$ & \\
\hline & & & & & & & & & & & $\begin{array}{l}\text { TwRS Initial Updated Retrieval } \\
\text { \& Disposal Baseline Summary }\end{array}$ & \\
\hline & & & & & & & & & & & $\begin{array}{l}\text { TWRS R\&DM Phase } 1 \\
\text { Financial Analysis }\end{array}$ & \\
\hline- & & & & - & $=$ & & & & & & Level 0 and Level 1 Logics & \\
\hline & & & & . & & & & & & & $\begin{array}{l}\text { TWRS R\&DM RTP } \\
\text { Memorandum }\end{array}$ & \\
\hline & & & & & 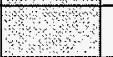 & & & & & & $\begin{array}{l}\text { Management Assessment of } \\
\text { TWRS RTP }\end{array}$ & \\
\hline & & & & & 1 & & & & & & \begin{tabular}{|l|} 
TWRS R\&DM RTP Internal \\
Indeoendent Assessment
\end{tabular} & \\
\hline
\end{tabular}




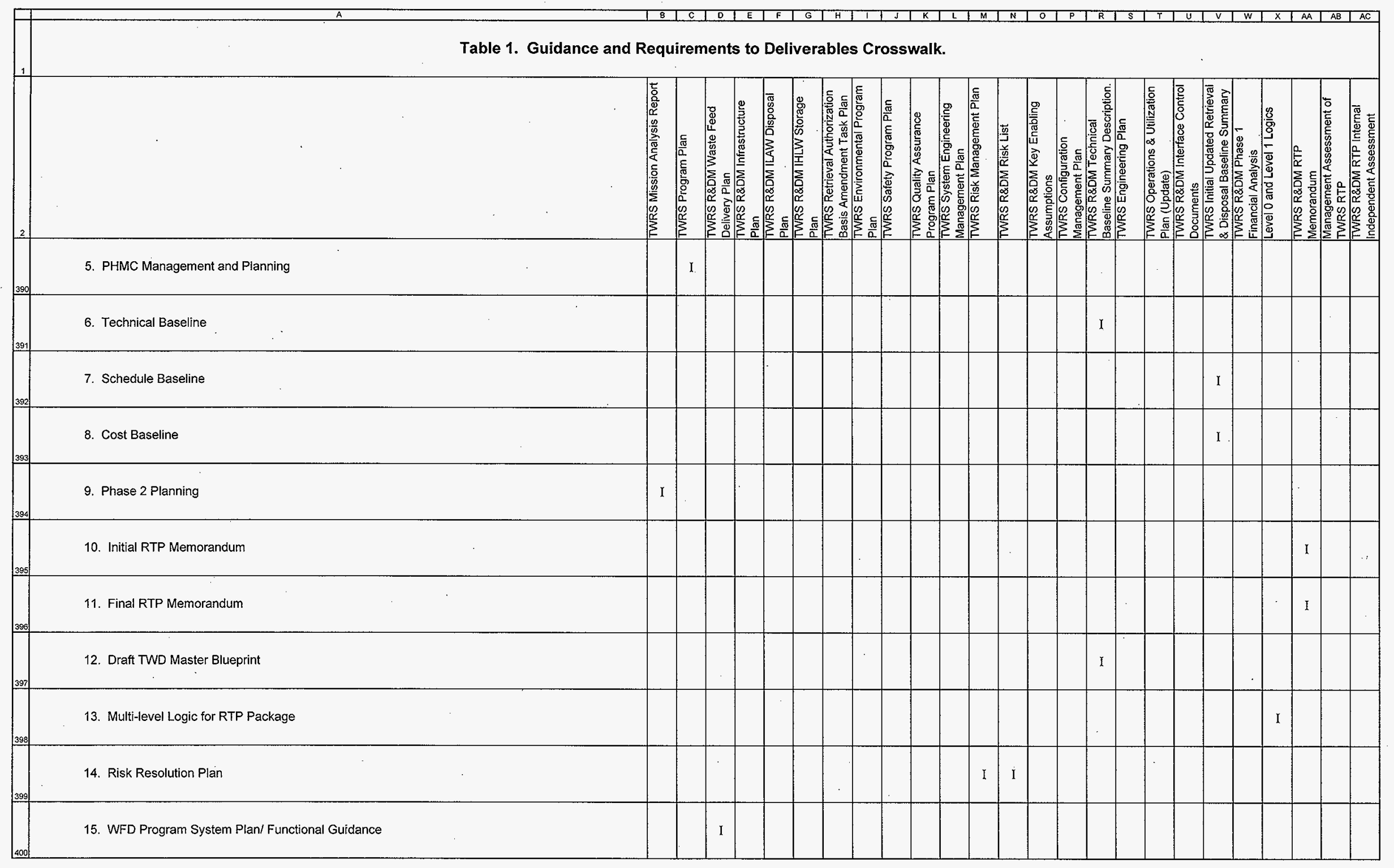




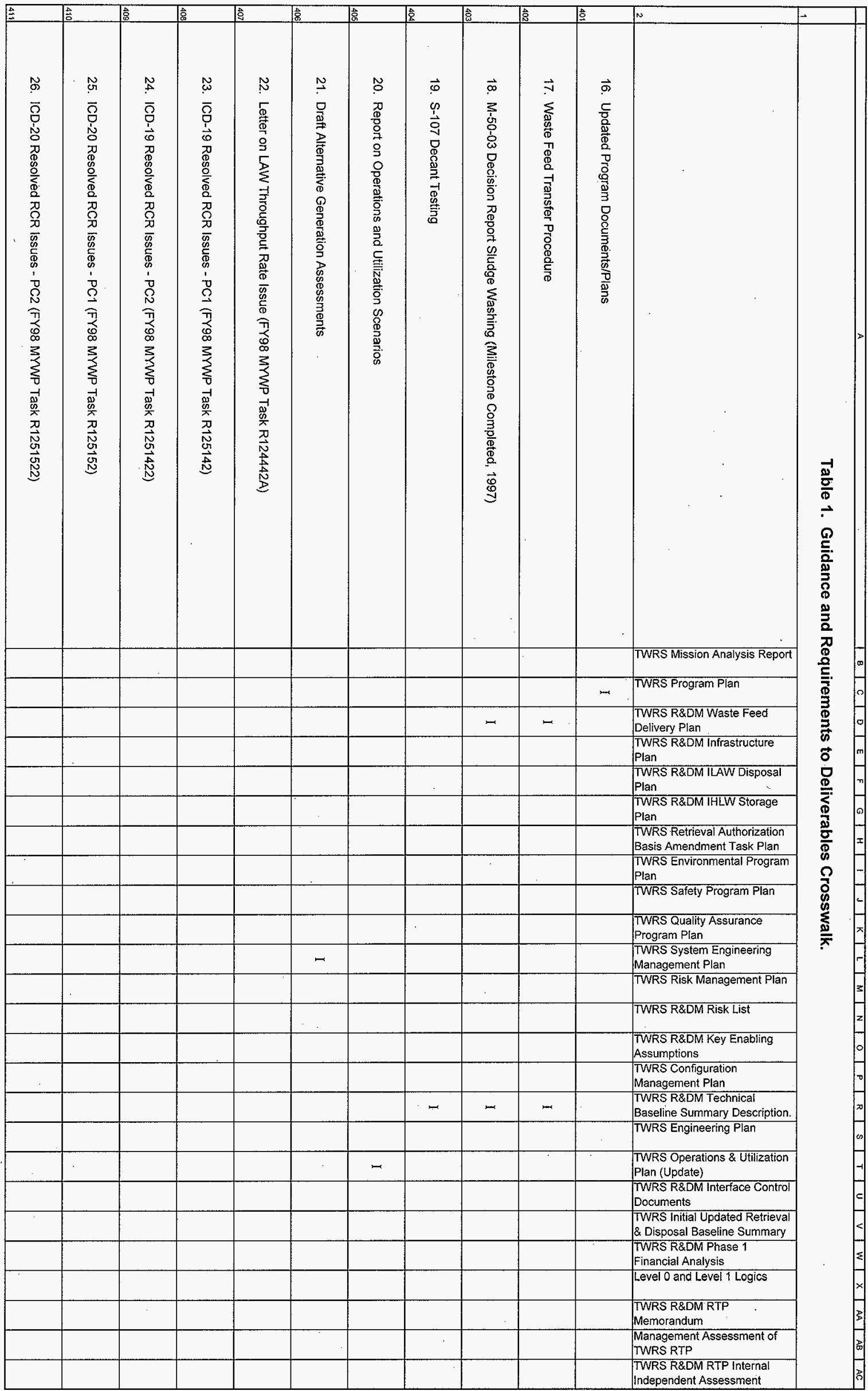




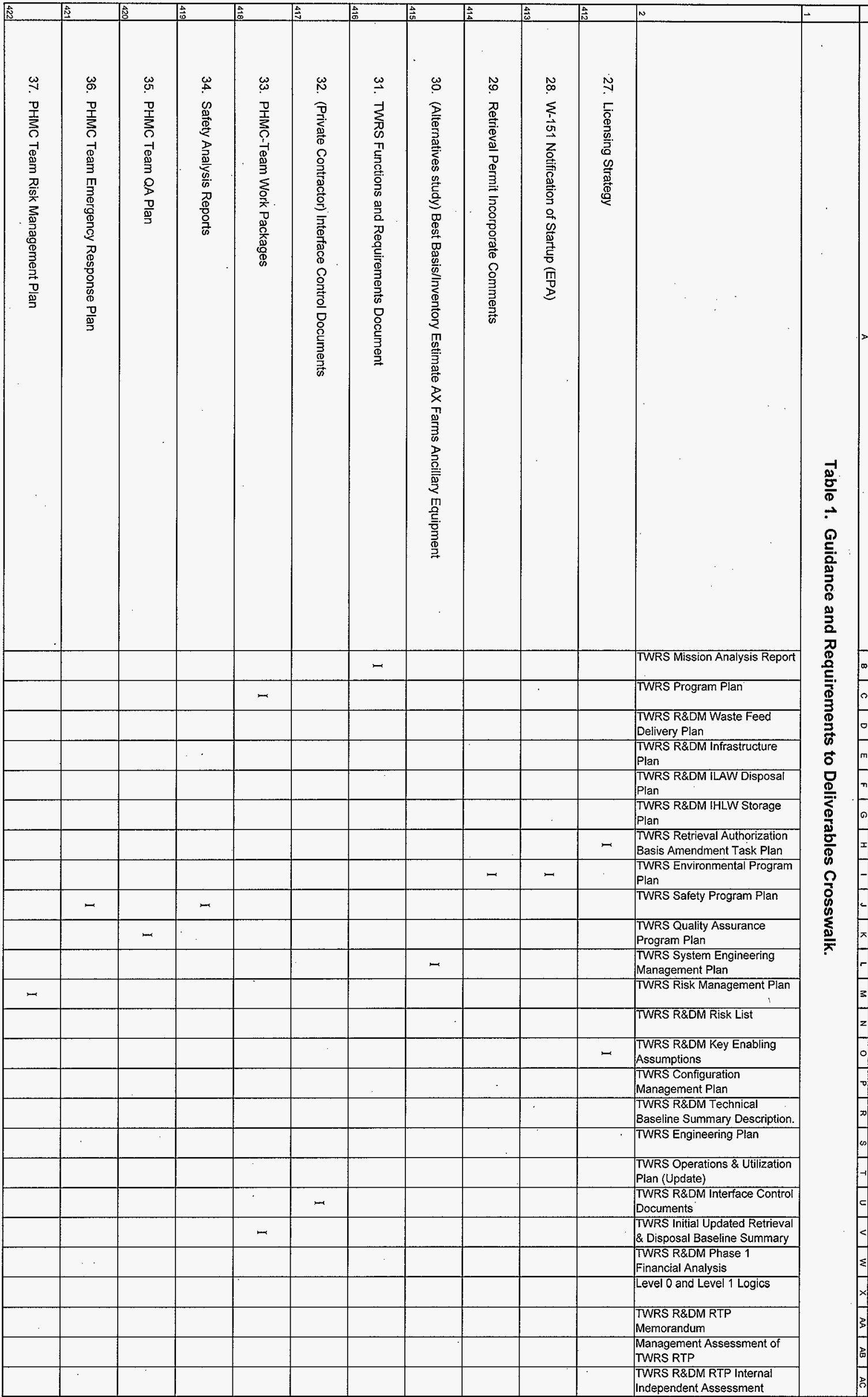




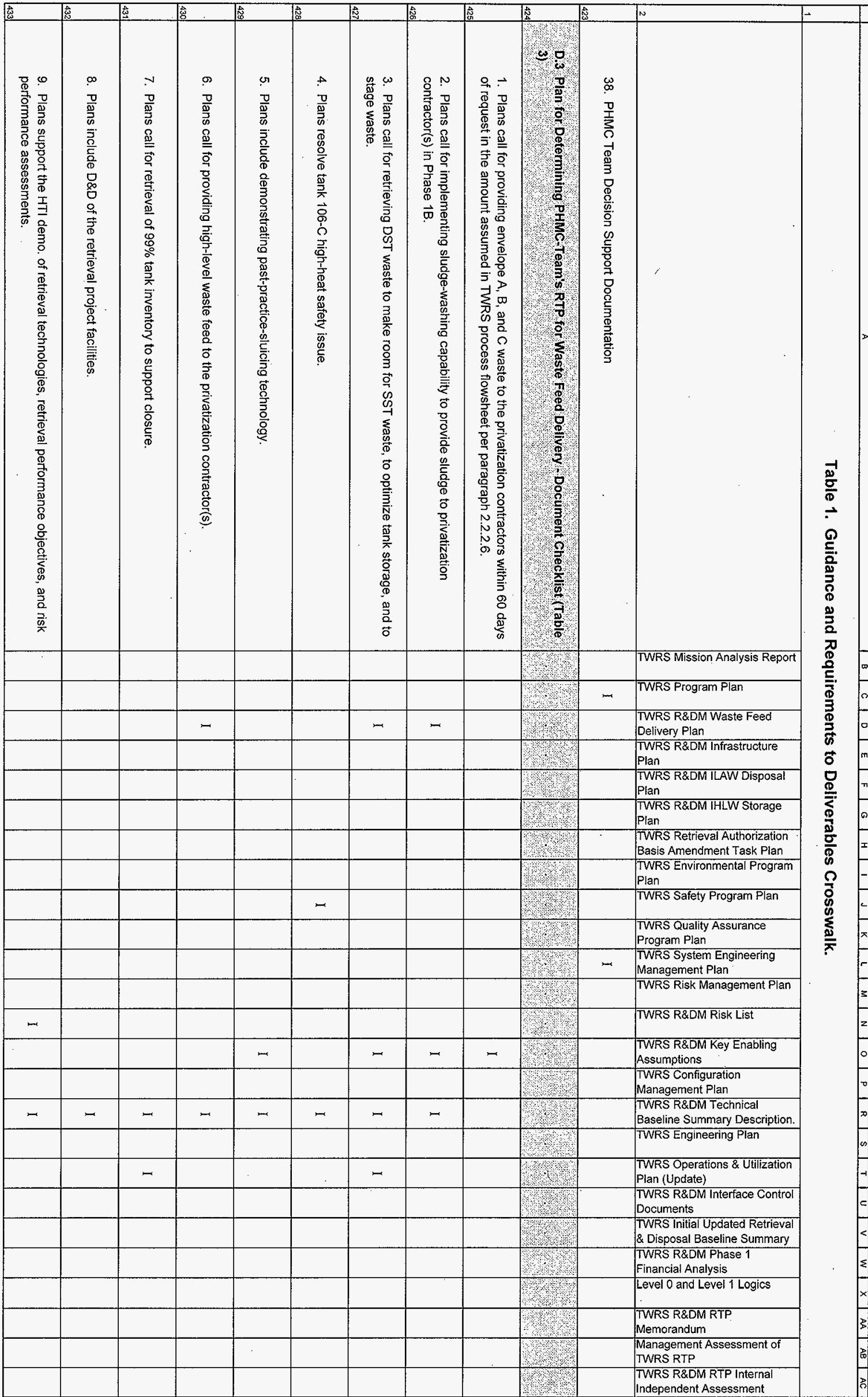




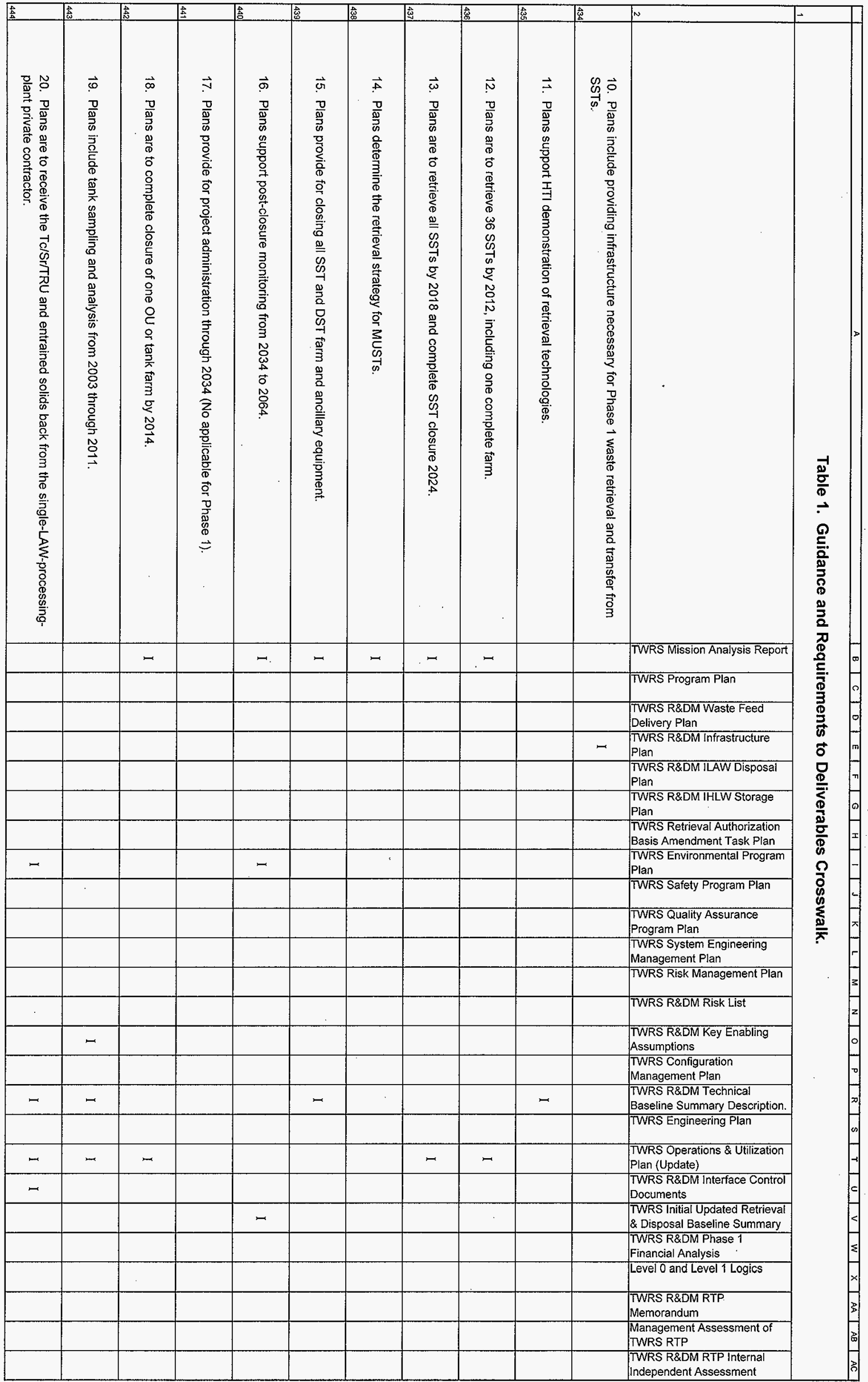




\begin{tabular}{|c|c|c|c|c|c|c|c|c|c|c|c|c|c|c|c|c|c|c|c|c|c|c|c|c|}
\hline 2 & & 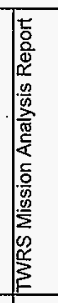 & 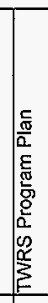 & 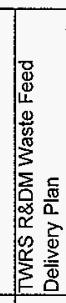 & 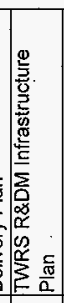 & 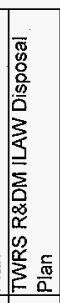 & 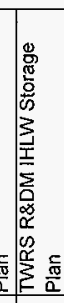 & 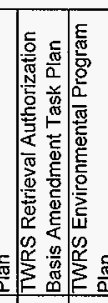 & 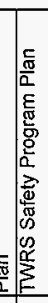 & 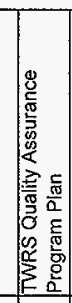 & 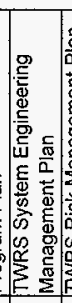 & 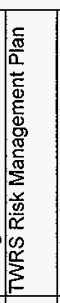 & 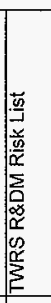 & 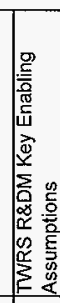 & 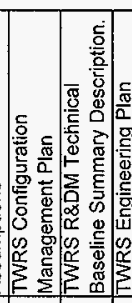 & & 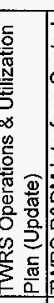 & 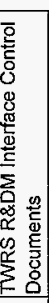 & 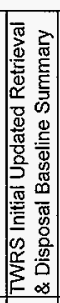 & 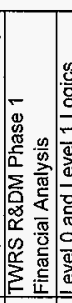 & 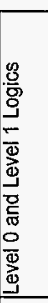 & 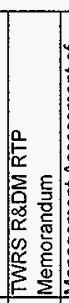 & 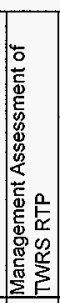 & 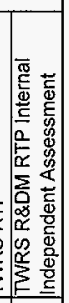 \\
\hline & 21. Plans are to perform acceptance testing of received $\mathrm{Tc} / \mathrm{S} r \mathrm{TRU}$. & & & & & & & & & I & & & & & I & & & I & & & & & & \\
\hline & 22. Plans are to arrange for delivery of TC/SrTRU back to a DST Tank Farm. & & & & & & & & & & & & & & & & I & & & & & & & \\
\hline 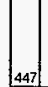 & $\begin{array}{l}\text { 23. Plans are to store separated TC, entrained HLW solids, and SrTRU products and collect costs of } \\
\text { this transport and storage. }\end{array}$ & & & & & & & & & & & & & & I & & I & & I & & & & & \\
\hline & 24. Plans are that TWR will be responsible for consolidation and feed staging for Phase 1 treatment. & & & I & & & & & & & & & & & & & & & & & & & & \\
\hline & $\begin{array}{l}\text { 25. Plans are that TWR will be responsible to ensure safe minimum ops of SST farms after SSTs are } \\
\text { interim-stabilized by } 2002 \text {. }\end{array}$ & & & & & & & & & & & & & & & & I & & & & & & & \\
\hline$l_{1500}$ & $\begin{array}{l}\text { 26. Plans are that TWR will be responsible for DST farm ops in FY06 and for S\&M of all farms for } \\
\text { which TWR assumes responsibility. }\end{array}$ & & & & & & & & & & & & & & & & I & & & & & & & \\
\hline & 27. Plans are for the PHMC to complete retrieval of at least one tank farm and 36 SSTs. & I & & & & & & & & & & & & & & & & & & & & & & \\
\hline & $\begin{array}{l}\text { 28. Plans are for the } 113 \text { remaining to be retrieved by a private contractor, who will assume } \\
\text { responsibility for ops and maintenance of those tank farms. }\end{array}$ & I & & & & & & & & & & & & & & & & & & & & & & \\
\hline & 29. Plans are to transfer DST ops to a private contractor in 2012. & I & & & & & & & & & & & & & & & & & & & & & & \\
\hline & $\begin{array}{l}\text { 30. Plans are for the 242-A evaporator to be supplied by the Liquid Effluents Program to support ops } \\
\text { until the immobilization facilities are in operation. }\end{array}$ & & & & & & & & & & & & & I & 1 & & I & & & & & & & \\
\hline & 31. Plans are for upgrades to the evaporator needed to extend ops. to 2011 to occur by 2005 . & & & & & & & & & & & & & I & & & & & I & & & & & \\
\hline
\end{tabular}




\begin{tabular}{|c|c|c|c|c|c|c|c|c|c|c|c|c|}
\hline & 咅 & 8 & 3 & & & & 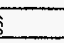 & & 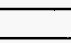 & 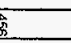 & $\omega$ & \\
\hline 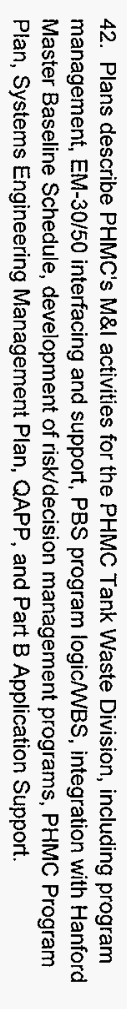 & 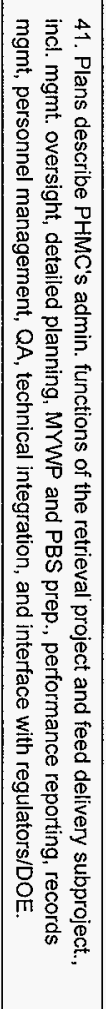 & 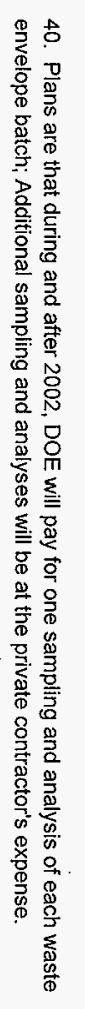 & 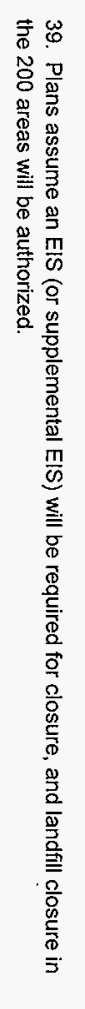 & 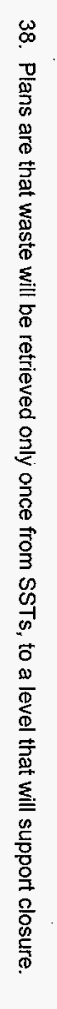 & 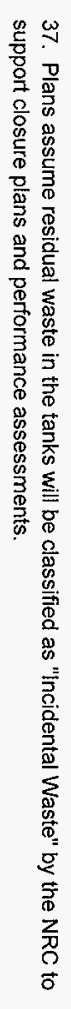 & 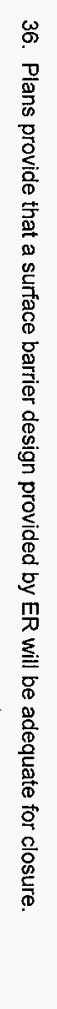 & 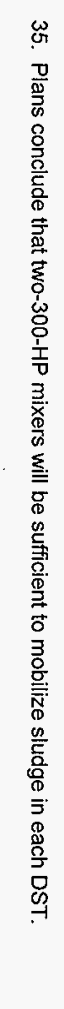 & 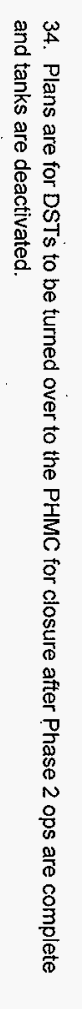 & 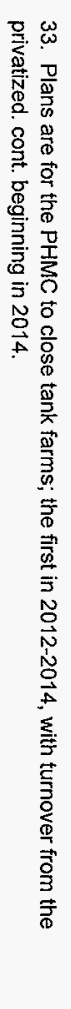 & 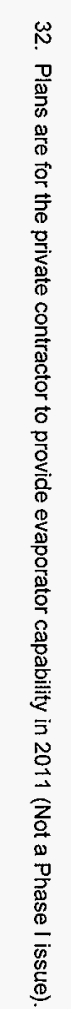 & . & 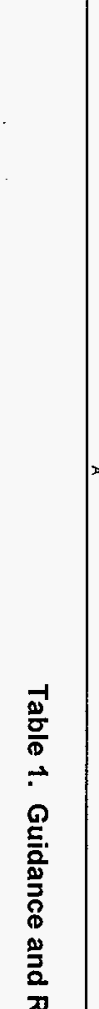 \\
\hline & & & & & & & & 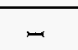 & $m$ & & TWRS Mission Analysis Report & 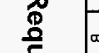 \\
\hline- & - & & & & & & & & & & TWRS Program Plan & $\overline{\bar{g}}$ \\
\hline & & & & & & & & & & & $\begin{array}{l}\text { TWRS R\&DM Waste Feed } \\
\text { Delivery Plan }\end{array}$ & $\stackrel{\overrightarrow{0}}{\vec{z}}$ \\
\hline & & & & & & & & & & & $\begin{array}{l}\text { TWRS R\&DM Infrastructure } \\
\text { Plan }\end{array}$ & $\frac{\infty}{0}$ \\
\hline & & & & & & & & & & & $\begin{array}{l}\text { TWRS R\&DM ILAW Disposal } \\
\text { Plan }\end{array}$ & $\underline{\underline{\Phi}}$ \\
\hline & & & & & & & & & & & $\begin{array}{l}\text { TWRS REDM IHLW Storage } \\
\text { Plan }\end{array}$ & 菖 \\
\hline- & & & & & & & & & & & \begin{tabular}{|l|} 
TWRS Retrieval Authorization \\
Basis Amendment Task Plan
\end{tabular} & $\frac{2}{0}$ \\
\hline & & & - & & - & & & & & & $\begin{array}{l}\text { TWRS Environmental Program } \\
\text { Plan }\end{array}$ & $\begin{array}{l}n \\
\Omega \\
\Omega\end{array}$ \\
\hline & & & & & & & & & & & TWRS Safety Program Plan & . \\
\hline- & - & & & & & & & & & & $\begin{array}{l}\text { TWRS Quality Assurance } \\
\text { Program Plan }\end{array}$ & 剂 \\
\hline - & & & & & & & & & & & $\begin{array}{l}\text { TWRS System Engineering } \\
\text { Management Plan }\end{array}$ & \\
\hline- & & & & & & & & & & & TWRS Risk Management Plan & \\
\hline & & & & & & & & & & & TWRS R\&DM Risk List & \\
\hline & & - & & & & & - & & & & $\begin{array}{l}\text { TWRS RRDM Key Enabling } \\
\text { Assumptions }\end{array}$ & \\
\hline & & & & & & & & & & & \begin{tabular}{|l} 
TWRS Configuration \\
Management Plan
\end{tabular} & \\
\hline & & & & - & - & & & & & & $\begin{array}{l}\text { TWRS R\&DM Technical } \\
\text { Baseline Summary Description }\end{array}$ & \\
\hline & & & & & & $r$ & m & & & & TWRS Engineering Plan & \\
\hline & & & & - & - & & & & & & $\begin{array}{l}\text { TWRS Operations \& Utilization } \\
\text { Plan (Update) }\end{array}$ & \\
\hline & & - & & & & & & & & & $\begin{array}{l}\text { TWRS R\&DM Interface Control } \\
\text { Documents }\end{array}$ & \\
\hline & & & & & & & & & & & \begin{tabular}{|l|} 
TWRS Initial Updated Retrieval \\
\& Disposal Baseline Summary
\end{tabular} & \\
\hline & & & & & & & & & & & $\begin{array}{l}\text { TWRS R\&DM Phase } 1 \\
\text { Financial Analysis }\end{array}$ & \\
\hline & & & & & & & & & & & Level 0 and Level 1 Logics & \\
\hline & & & & & & & & & & & $\begin{array}{l}\text { TWRS R\&DM RTP } \\
\text { Memorandum }\end{array}$ & \\
\hline & & & & & & & & & & & $\begin{array}{l}\text { Management Assessment of } \\
\text { TWRS RTP }\end{array}$ & \\
\hline & & & & & & & & & & & $\begin{array}{l}\text { TWRS R\&DM RTP Internal } \\
\text { Independent Assessment }\end{array}$ & \\
\hline
\end{tabular}




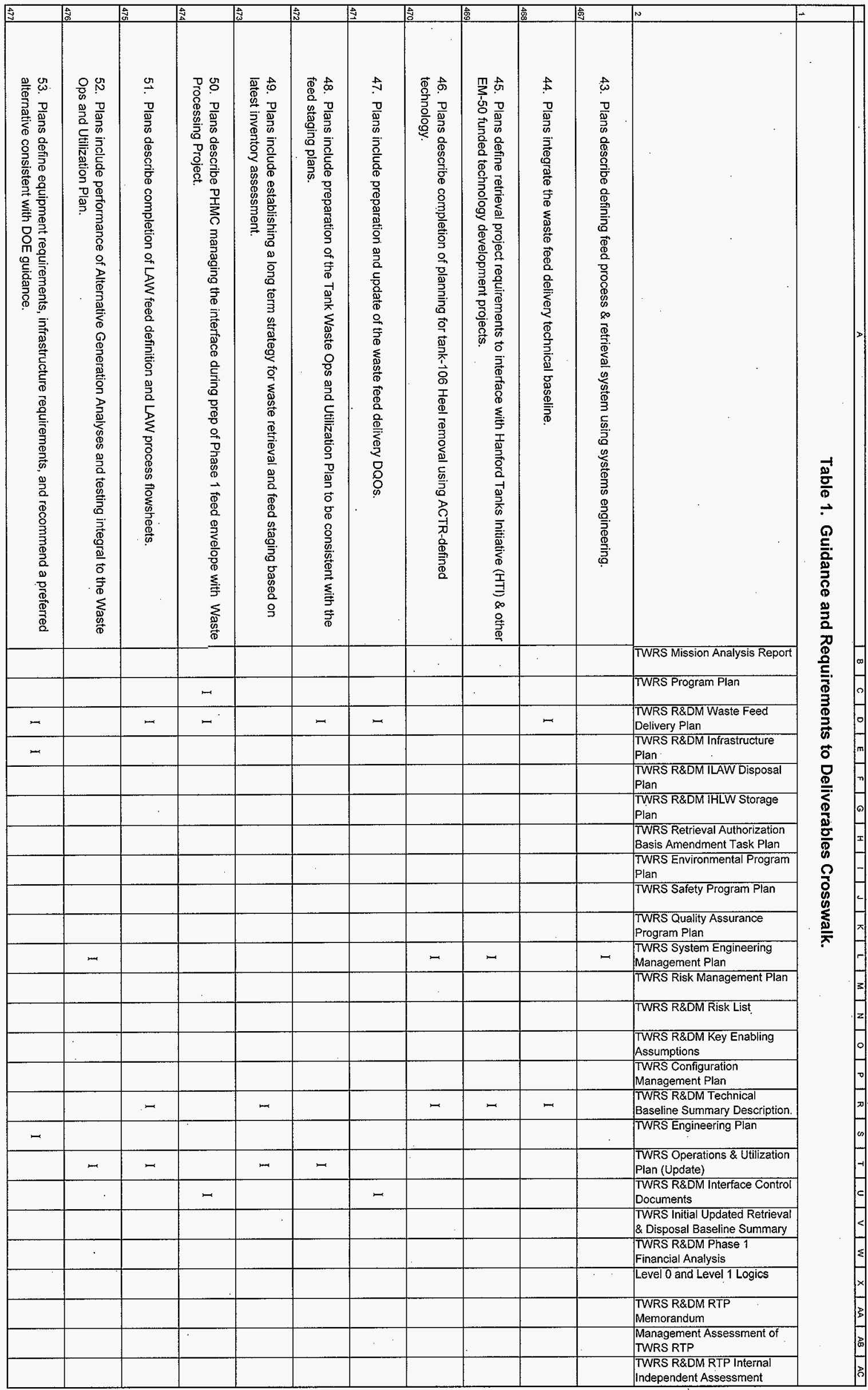




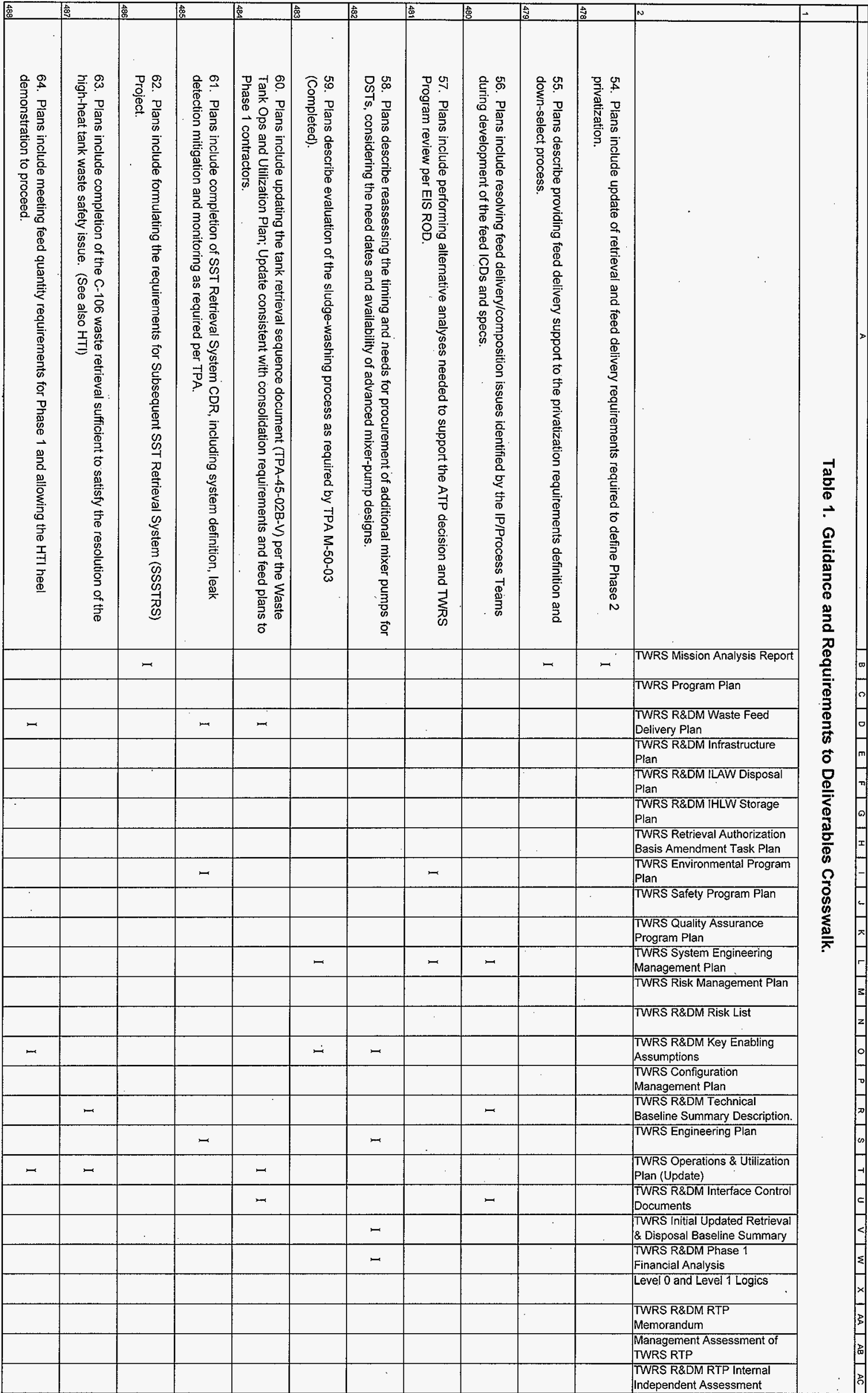




\begin{tabular}{|c|c|c|c|c|c|c|c|c|c|c|c|c|}
\hline & & & & & & & 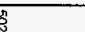 & & & & $T_{N}$ & \\
\hline 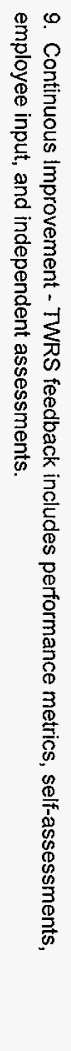 & 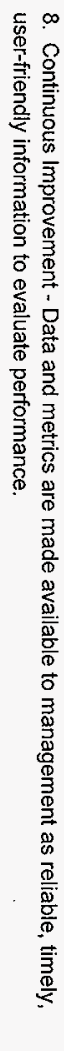 & 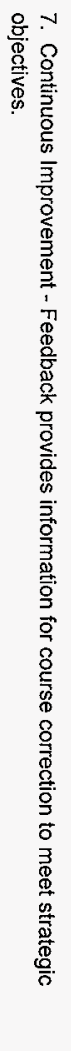 & 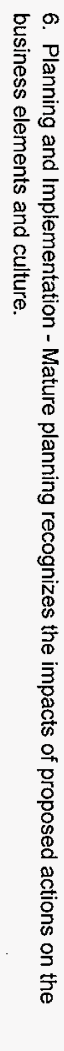 & 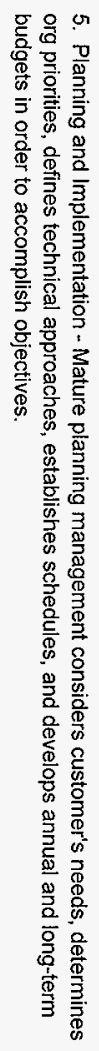 & 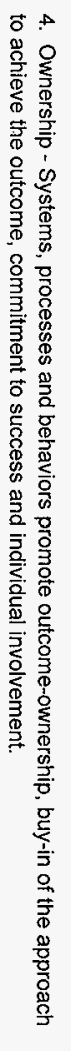 & 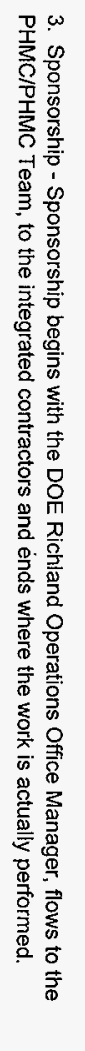 & 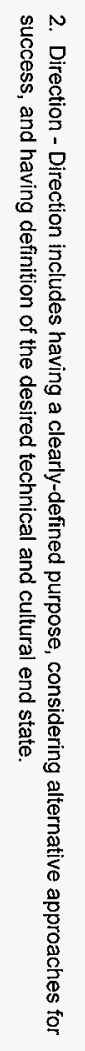 & 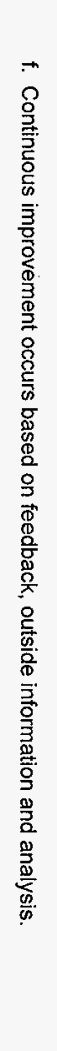 & 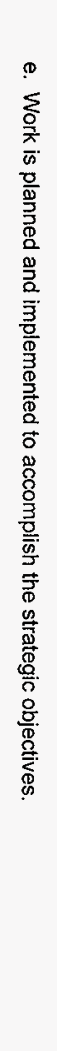 & 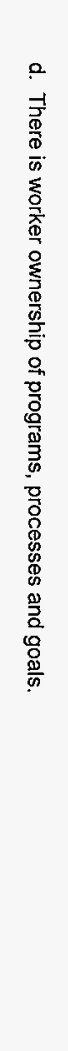 & & 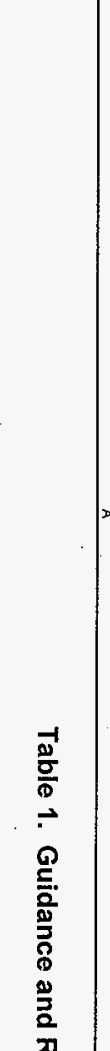 \\
\hline & & & & & & & & & & & TWRS Mission Analysis Report & 苾 \\
\hline & & - & - & - & - & - & - & - & - & & TWRS Program Plan & $\overline{\bar{\Phi}}$ \\
\hline & & r & & & & & & & & & $\begin{array}{l}\text { TWRS R\&DM Waste Feed } \\
\text { Delivery Plan }\end{array}$ & 帝 \\
\hline & & & & & & & & & & & $\begin{array}{l}\text { TWRS R\&DM Infrastructure } \\
\text { Plan }\end{array}$ & के \\
\hline & & . & & & & & & & & & $\begin{array}{l}\text { TWRS R\&DM ILAW Disposal } \\
\text { Plan }\end{array}$ & $\underset{\underline{\Phi}}{\underline{\underline{p}}}$ \\
\hline & & & & & & & & & & & $\begin{array}{l}\text { TWRS R\&DM IHLW Storage } \\
\text { Plan }\end{array}$ & స్త \\
\hline & & & & & & & & & & & \begin{tabular}{|l|} 
TWRS Retrieval Authorization \\
Basis Amendment Task Plan \\
\end{tabular} & $\frac{\tilde{\sigma}}{\frac{\sigma}{0}}$ \\
\hline & & & & & & & & & & & $\begin{array}{l}\text { TWRS Environmental Program } \\
\text { Plan }\end{array}$ & $\begin{array}{l}0 \\
0 \\
0\end{array}$ \\
\hline & & & & & & & & & & & TWRS Safety Program Plan & 足 \\
\hline$=$ & - & $=$ & & & & & & & & & \begin{tabular}{|l} 
TWRS Quality Assurance \\
Program Plan
\end{tabular} & 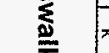 \\
\hline & & & & & & & & & & & $\begin{array}{l}\text { TWRS System Engineering } \\
\text { Management Plan }\end{array}$ & \\
\hline & & & & & & & & & & & TWRS Risk Management Plan & \\
\hline & & & & & & & & & & & TWRS R\&DM Risk List & \\
\hline & & & & & & & & & & & $\begin{array}{l}\text { TWRS R2DM Key Enabling } \\
\text { Assumptions }\end{array}$ & \\
\hline & & & & & & & & & & & $\begin{array}{l}\text { TWRS Configuration } \\
\text { Management Plan }\end{array}$ & \\
\hline & & & & & & & & & & & $\begin{array}{l}\text { TWRS RQDM Technical } \\
\text { Baseline Summary Description. }\end{array}$ & \\
\hline & & & & & & & & & & & TWRS Engineering Plan & \\
\hline & & & & & & & & & & & $\begin{array}{l}\text { TWRS Operations \& Utilization } \\
\text { Plan (Update) }\end{array}$ & \\
\hline & & & & & & & & & & & \begin{tabular}{|l|} 
TWRS R2DM Interface Control \\
Documents
\end{tabular} & \\
\hline & & & & & & & & & & & $\begin{array}{l}\text { TWRS Initial Updated Retrieval } \\
\text { \& Disposal Baseline Summary }\end{array}$ & \\
\hline & & & & & & & & & & & $\begin{array}{l}\text { TWRS R\&DM Phase } 1 \\
\text { Financial Analysis }\end{array}$ & \\
\hline & & & & & & & & & & & Level 0 and Level 1 Logics & \\
\hline & & & & & - & - & & & & & $\begin{array}{l}\text { TWRS R\&DM RTP } \\
\text { Memorandum }\end{array}$ & \\
\hline- & - & - & & & 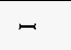 & 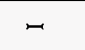 & $m$ & - & - & - & $\begin{array}{l}\text { Management Assessment of } \\
\text { TWRRS RTP }\end{array}$ & \\
\hline & & & & & & & & & & & \begin{tabular}{|l} 
WWRS R\&DM RTP Internal \\
Independent Assessment
\end{tabular} & \\
\hline
\end{tabular}




\begin{tabular}{|c|c|c|c|c|c|c|c|c|c|c|c|}
\hline & 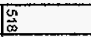 & $\Xi$ & $\frac{5}{5}$ & $\frac{9}{2}$ & $\frac{\underline{n}}{\underline{\underline{n}}}$ & 要 & 篦 & $\frac{2}{2}$ & $\frac{200}{0}$ & $N$ & - \\
\hline 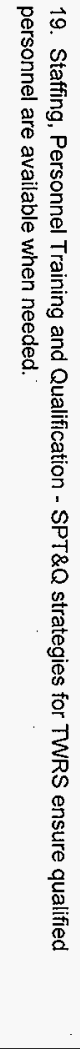 & 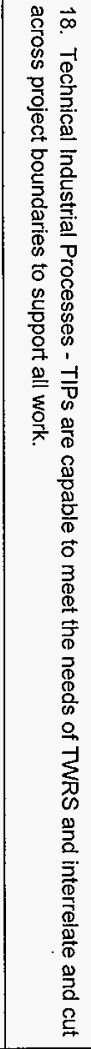 & 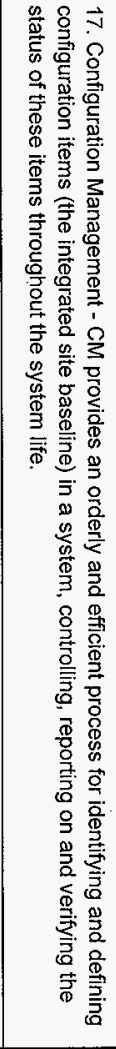 & 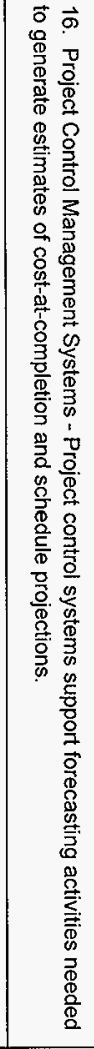 & 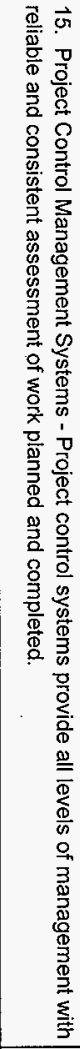 & 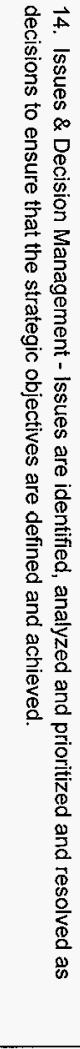 & 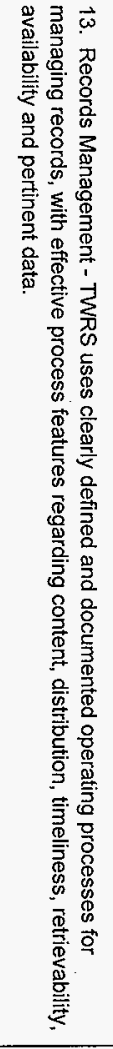 & 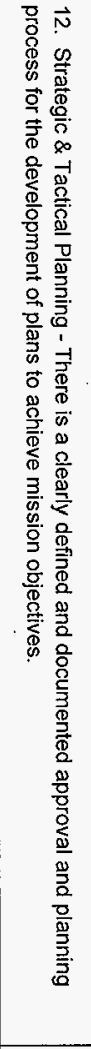 & 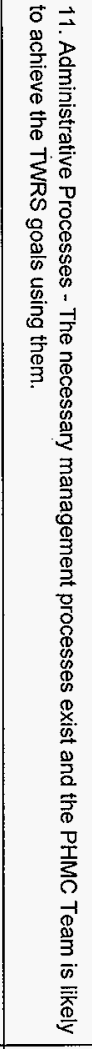 & 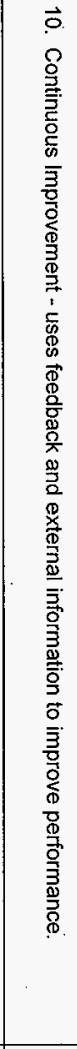 & 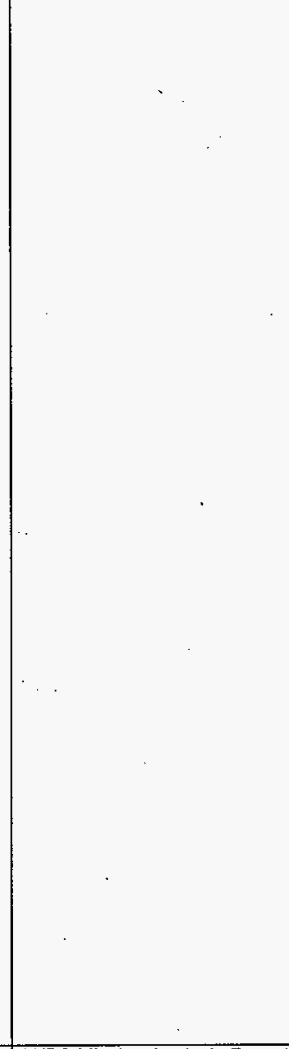 & 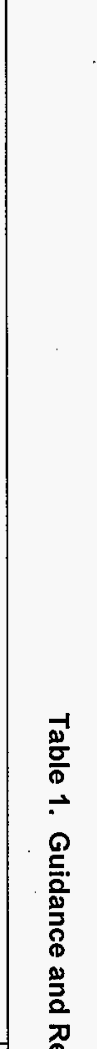 \\
\hline & & & & & & & - & & & TWRS Mission Analysis Report & $\stackrel{D}{2}$ \\
\hline- & & - & - & $m$ & - & $\varpi$ & & - & & TWRS Program Plan & $\overline{\mathbb{D}}$ \\
\hline & & & & & & & & & & $\begin{array}{l}\text { TWRS R\&DM Waste Feed } \\
\text { Delivery Plan }\end{array}$ & $\stackrel{0}{2}$ \\
\hline & & & & & & & & & & $\begin{array}{l}\text { TWRS R\&DM Infrastructure } \\
\text { Plan }\end{array}$ & is \\
\hline & & & & & & & & & & $\begin{array}{l}\text { TWRS R\&DM ILAW Disposal } \\
\text { Plan }\end{array}$ & 足 \\
\hline & & & & & & & & & & $\begin{array}{l}\text { TWRS R\&DM IHLW Storage } \\
\text { Plan }\end{array}$ & হ゙ \\
\hline & & & & & & & & & & $\begin{array}{l}\text { TWRS Retrieval Authorization } \\
\text { Basis Amendment Task Plan }\end{array}$ & $\frac{20}{\frac{0}{D}}$ \\
\hline & & & & & & & & & & $\begin{array}{l}\text { TWRS Environmental Program } \\
\text { Plan }\end{array}$ & @ \\
\hline & & & & & & & & & & TWRS Safety Program Plan & \\
\hline & & & & & & & & & $m$ & $\begin{array}{l}\text { TWRS Quality Assurance } \\
\text { Program Plan }\end{array}$ & 惡 \\
\hline & & & & & & & & & & $\begin{array}{l}\text { TWRS System Engineering } \\
\text { Management Plan }\end{array}$ & \\
\hline & & & & & & & & & & TWRS Risk Management Plan & \\
\hline & & & & & & & & & & TWRS R\&DM Risk List & \\
\hline & & & & & & & & & & $\begin{array}{l}\text { TWRS R\&DM Key Enabling } \\
\text { Assumptions }\end{array}$ & \\
\hline & & 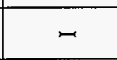 & & & & - & & & & $\begin{array}{l}\text { TWRS Configuration } \\
\text { Management Plan }\end{array}$ & \\
\hline & & & & & & & & & & $\begin{array}{l}\text { TWRS R\&DM Technical } \\
\text { Baseline Summary Description. }\end{array}$ & \\
\hline & & & & & & & & & & TWRS Engineering Plan & \\
\hline & & & & & & & & & & $\begin{array}{l}\text { TWRS Operations \& Utilization } \\
\text { Plan (Update) }\end{array}$ & \\
\hline & & & & & & & & & & $\begin{array}{l}\text { TWRS R\&DM Interface Control } \\
\text { Documents }\end{array}$ & \\
\hline & & & & & & & & & & $\begin{array}{l}\text { TWRS Initial Updated Retrieval } \\
\text { \& Disposal Baseline Summary }\end{array}$ & \\
\hline & & & & & & & & & & $\begin{array}{l}\text { TWRS R\&DM Phase } 1 \\
\text { Financial Analysis }\end{array}$ & \\
\hline & & & & & & & & & & Level 0 and Level 1 Logics & \\
\hline & & & & & & & & & & $\begin{array}{l}\text { TWRS R\&DM RTP } \\
\text { Memorandum }\end{array}$ & \\
\hline- & - & & & & - & & & - & & $\begin{array}{l}\text { Management Assessment of } \\
\text { TWRS RTP }\end{array}$ & \\
\hline & & & & & & & & & & $\begin{array}{l}\text { TWRS R\&DM RTP Internal } \\
\text { Independent Assessment }\end{array}$ & \\
\hline
\end{tabular}




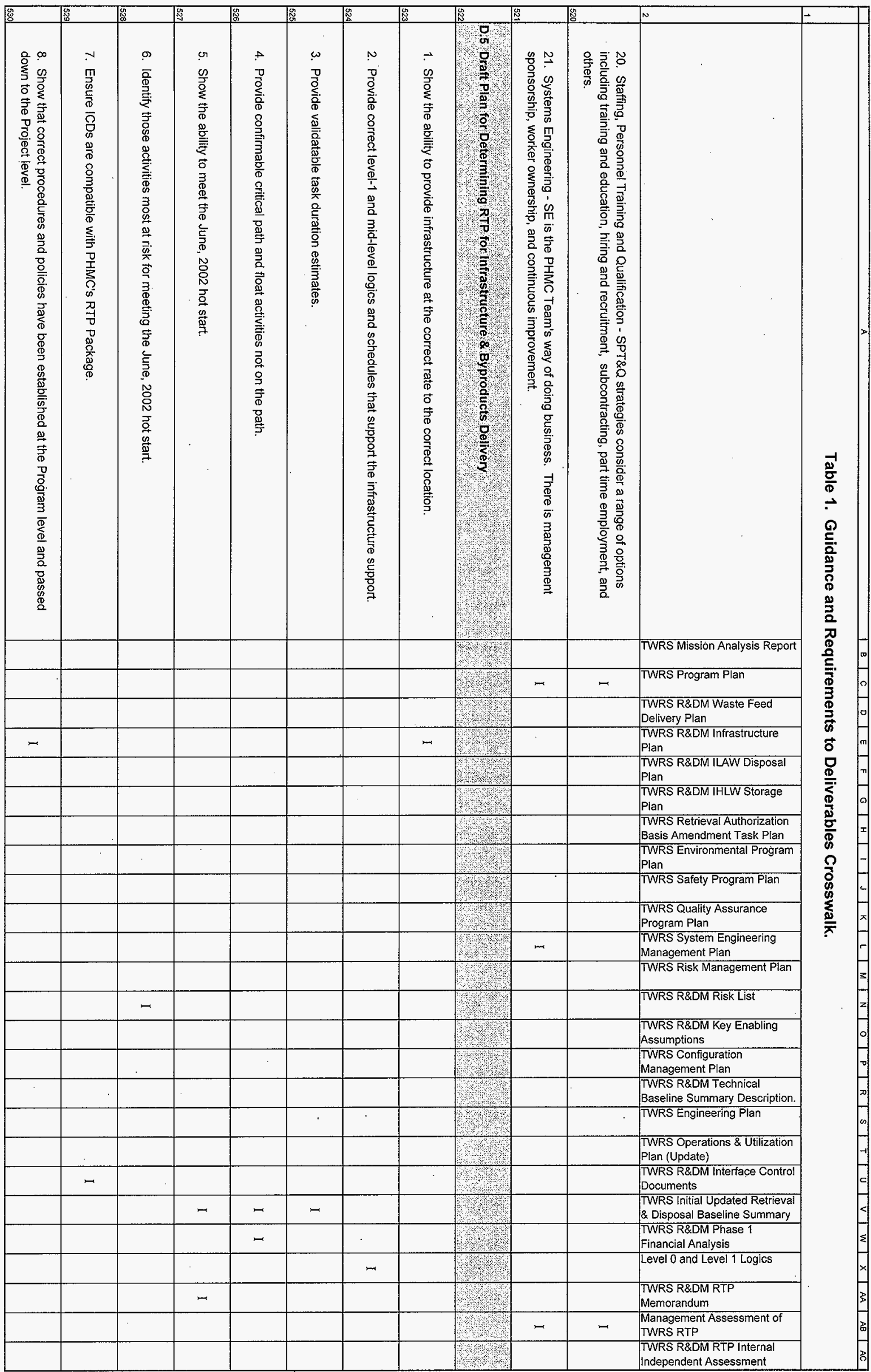




\begin{tabular}{|c|c|c|c|c|c|c|c|c|c|c|c|c|}
\hline \multirow{5}{*}{ 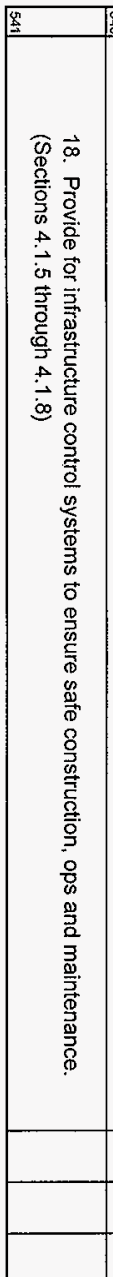 } & . & 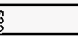 & . & 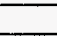 & 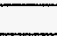 & . & 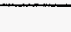 & & & & N & \\
\hline & 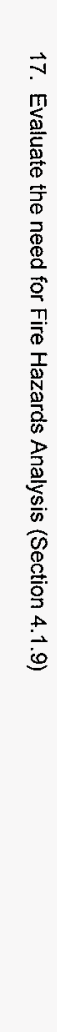 & 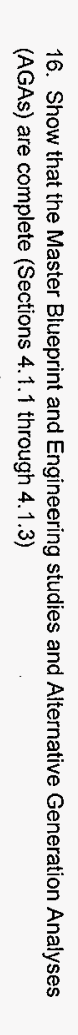 & 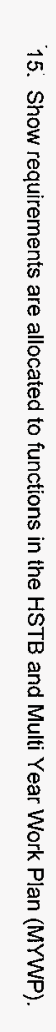 & 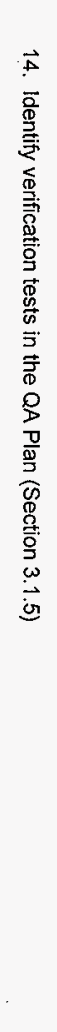 & 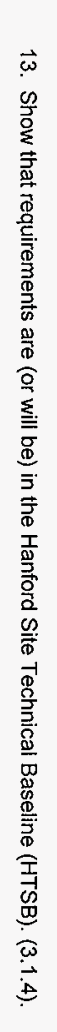 & 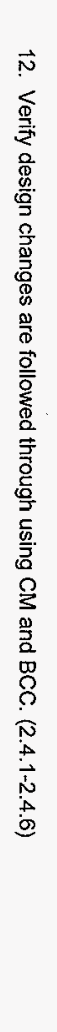 & 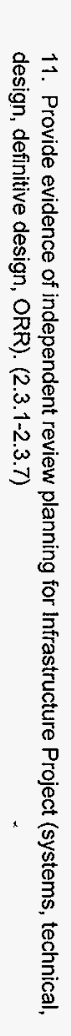 & 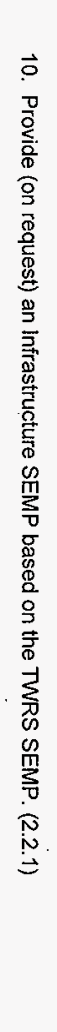 & 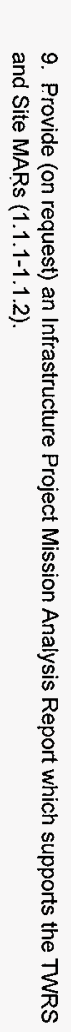 & 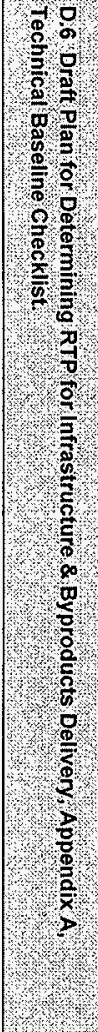 & 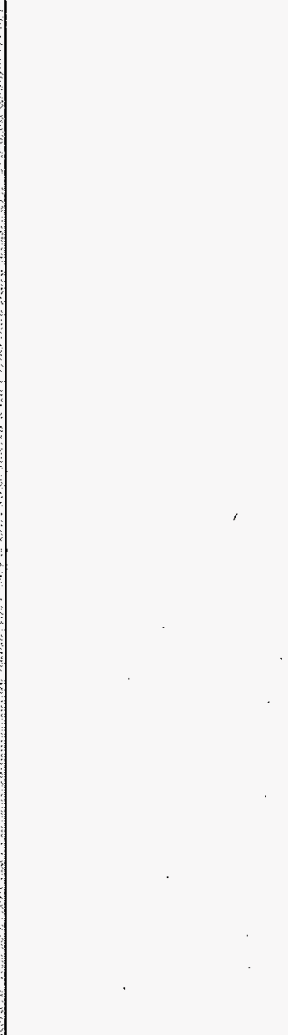 & 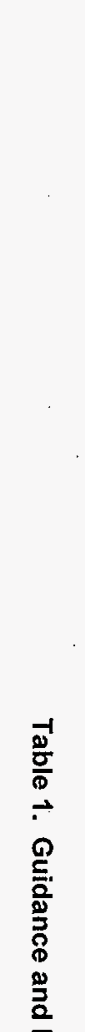 \\
\hline & & & $m$ & - & -7 & & & & 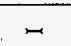 & & TWRS Mission Analysis Report & $\stackrel{8}{z}$ \\
\hline & & & & & & & & & & & TWRS Program Plan & $\Phi$ \\
\hline & & & & & & & & & & & $\begin{array}{l}\text { TWRS R\&DM Waste Feed } \\
\text { Delivery Plan }\end{array}$ & 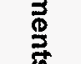 \\
\hline- & & & & & & & - & & & & $\begin{array}{l}\text { TWRS R\&DM Infrastructure } \\
\text { Plan }\end{array}$ & $\begin{array}{l}\bar{\sigma} \\
0 \\
0\end{array}$ \\
\hline & & & & & & & & & & & $\begin{array}{l}\text { TWRS R\&DM ILAW Disposal } \\
\text { Plan }\end{array}$ & $\underset{\Phi}{\mathscr{\Phi}}$ \\
\hline & & & & & & & & & & & $\begin{array}{l}\text { TWRS R\&DM IHLW Storage } \\
\text { Plan }\end{array}$ & $\bar{\S}$ \\
\hline & & & & & & & & & & & $\begin{array}{l}\text { TWRS Retrieval Authorization } \\
\text { Basis Amendment Task Plan }\end{array}$ & $\frac{\pi}{8}$ \\
\hline & & & & & & & & & & & $\begin{array}{l}\text { TWRS Environmental Program } \\
\text { Plan }\end{array}$ & 菓 \\
\hline- & - & & & & & & - & & & & TWRS Safety Program Plan & 8 \\
\hline & & & & - & & & & & & & $\begin{array}{l}\text { TWRS Quality Assurance } \\
\text { Program Plan }\end{array}$ & 总 \\
\hline & & & & & & & & - & & & $\begin{array}{l}\text { TWRS System Engineering } \\
\text { Management Plan }\end{array}$ & \\
\hline & & & & & & & & & & & TWRS Risk Management Plan & \\
\hline & & & & & & & & & & & TWRS R\&DM Risk List & \\
\hline & & & & & & & & & & & $\begin{array}{l}\text { TWRS R\&DM Key Enabling } \\
\text { Assumpitions }\end{array}$ & \\
\hline & & & & & & - & & & & & $\begin{array}{l}\text { TWRS Configuration } \\
\text { Management Plan }\end{array}$ & \\
\hline - & & & & & & & & & & & $\begin{array}{l}\text { TWRS R\&DM Technical } \\
\text { Baseline Summary Description. }\end{array}$ & \\
\hline & & - & & & & & & & & & TWRS Engineering Plan & \\
\hline & & & & & & & & & & & $\begin{array}{l}\text { TWRS Operations \& Utilization } \\
\text { Plan (Update) }\end{array}$ & \\
\hline & & & & & & & & & & & $\begin{array}{l}\text { TWRS R\&DM Interface Control } \\
\text { Documents }\end{array}$ & \\
\hline & & & & & & & & & & & $\begin{array}{l}\text { TWRS Initial Updated Retrieval } \\
\text { \& Disposal Baseline Summary } \\
\end{array}$ & \\
\hline & & & & & & & & & & & $\begin{array}{l}\text { TWRS R\&DM Phase } 1 \\
\text { Financial Analysis }\end{array}$ & \\
\hline & & & & & & & & & & & Level 0 and Level 1 Logics & \\
\hline & & & & & & & & & & & \begin{tabular}{|l} 
TWRS R\&DM RTP \\
Memorandum
\end{tabular} & \\
\hline & & & & & & & & & & & $\begin{array}{l}\text { Management Assessment of } \\
\text { JWRS RTP }\end{array}$ & \\
\hline & & & & & & & & & & $t$ & $\begin{array}{l}\text { TWRS R\&DM RTP Internal } \\
\text { Independent Assessment }\end{array}$ & \\
\hline
\end{tabular}




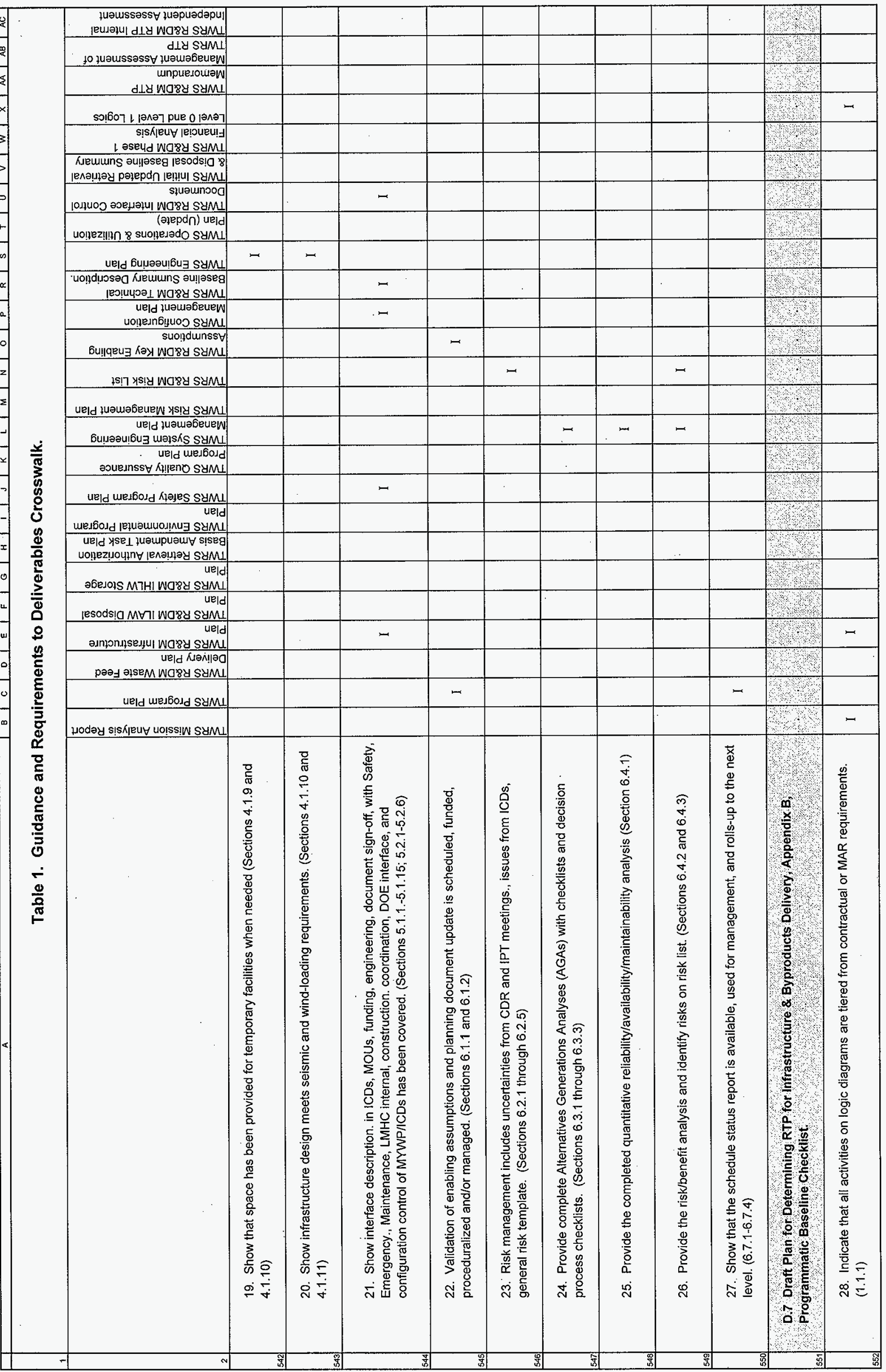




\begin{tabular}{|c|c|c|c|c|c|c|c|c|c|c|c|c|}
\hline & 3 & & & & & & 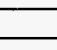 & 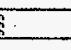 & 要 & & $N$ & 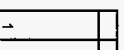 \\
\hline 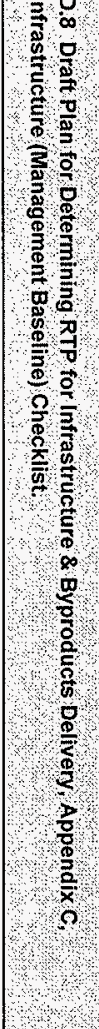 & 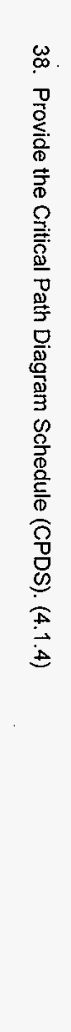 & 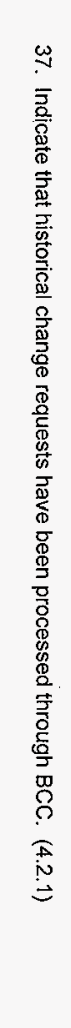 & 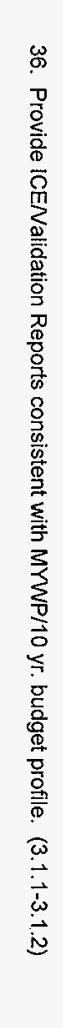 & 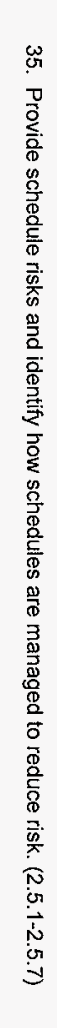 & 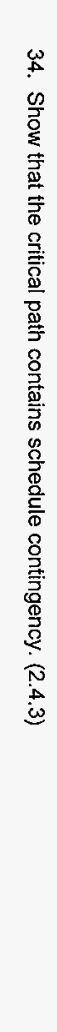 & 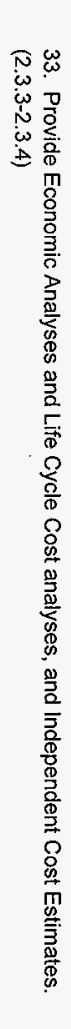 & 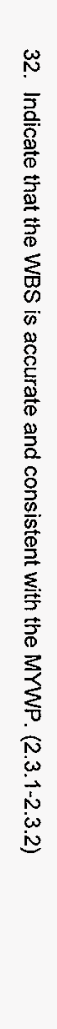 & 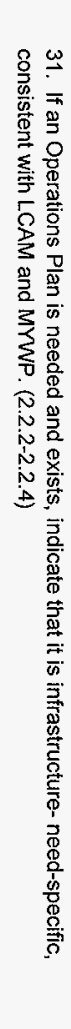 & 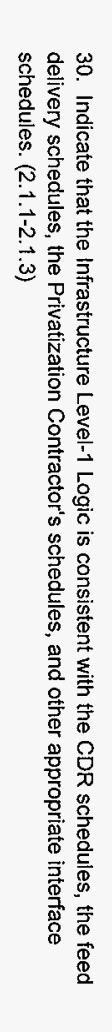 & 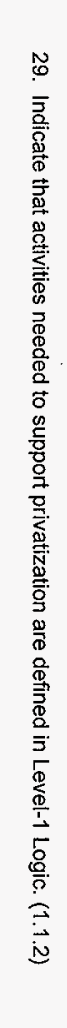 & & 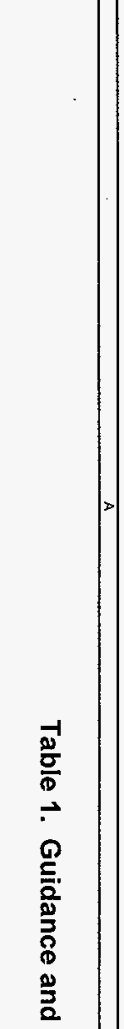 \\
\hline & & & & & & & & & & & TWRS Mission Analysis Report & $\begin{array}{l}\text { Do } \\
\text { D. } \\
=\end{array}$ \\
\hline & & & & - & & & & & & & TWRS Program Plan & 弯 \\
\hline & & & & & & & & & & & $\begin{array}{l}\text { TWRS R\&DM Waste Feed } \\
\text { Delivery Plan }\end{array}$ & $\stackrel{0}{=}$ \\
\hline & & & & & & & - & - & - & & $\begin{array}{l}\text { TWRS R\&DM Infrastructure } \\
\text { Plan }\end{array}$ & $\overrightarrow{0}$ \\
\hline & & & & & & & & & & & $\begin{array}{l}\text { WWRS R\&DM ILAW Disposal } \\
\text { Plan }\end{array}$ & $\underset{D}{\mathscr{D}}$ \\
\hline & & & & & & & & & & & $\begin{array}{l}\text { TWRS R\&DM IHLW Storage } \\
\text { Plan }\end{array}$ & 离 \\
\hline+8 & & & & & & & & & & & $\begin{array}{l}\text { TWRS Retrieval Authorization } \\
\text { Basis Amendment Task Plan }\end{array}$ & $\frac{\frac{3}{0}}{\frac{2}{0}}$ \\
\hline & & & & & & & & & & & $\begin{array}{l}\text { TWRS Environmental Program } \\
\text { Plan }\end{array}$ & 足 \\
\hline & & & & & & & & & & & TWRS Safety Program Plan & 8 \\
\hline & & & & & & & & & & & $\begin{array}{l}\text { TWRS Quality Assurance } \\
\text { Program Plan }\end{array}$ & 产 \\
\hline & & & & & & & & & & & $\begin{array}{l}\text { TWRS System Engineering } \\
\text { Management Plan }\end{array}$ & \\
\hline & & & & - & & & & & & & TWRS Risk Management Plan & \\
\hline 4 & & & & - & & & & & & & TWRS R\&DM Risk List & \\
\hline & & & & - & & & & & & & $\begin{array}{l}\text { TWRS R\&DM Key Enabling } \\
\text { Assumptions }\end{array}$ & \\
\hline & & m & & & & & & & & & $\begin{array}{l}\text { TWRS Configuration } \\
\text { Management Plan }\end{array}$ & \\
\hline & & & & & & & & & & & $\begin{array}{l}\text { TWRS R\&DM Technical } \\
\text { Baseline Summary Description. }\end{array}$ & \\
\hline & & & & & & & & & & & TWRS Engineering Plan & \\
\hline & & & & & & & & & & & TWRS Operations \& Utilization & \\
\hline 3 & & & & & & & & & & & U Interface Control & \\
\hline 3 & - & & & & - & & - & & - & & $\begin{array}{l}\text { TWRS Initial Uddated Retrieval } \\
\text { \& Disposal Baseline Summary }\end{array}$ & \\
\hline 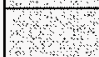 & & & & - & & - & & & & & $\begin{array}{l}\text { TWRS R\&DM Phase } 1 \\
\text { Financial Analysis }\end{array}$ & \\
\hline 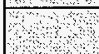 & & & & & & & & & - & - & Level 0 and Level 1 Logics & \\
\hline . & & & & & & & & & & & $\begin{array}{l}\text { TWRS R\&DM RTP } \\
\text { Memorandum }\end{array}$ & \\
\hline \% & & & & & & & & & & & $\begin{array}{l}\text { Management Assessment of } \\
\text { TWRS RTP }\end{array}$ & \\
\hline 2 & & & - & & & - & & & & & $\begin{array}{l}\text { TWRSR R\&DM RTP Internal } \\
\text { Independent Assessment }\end{array}$ & \\
\hline
\end{tabular}




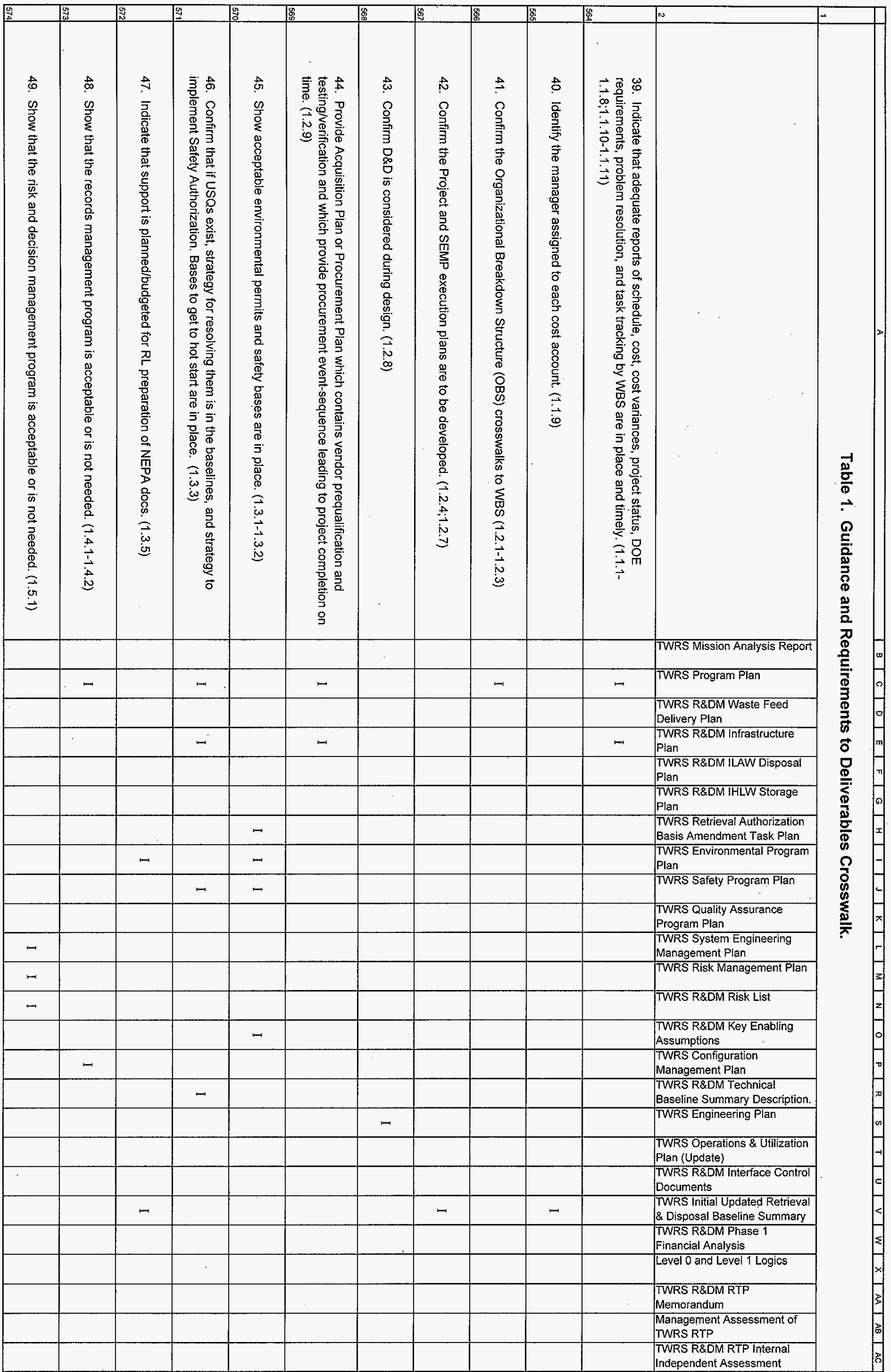




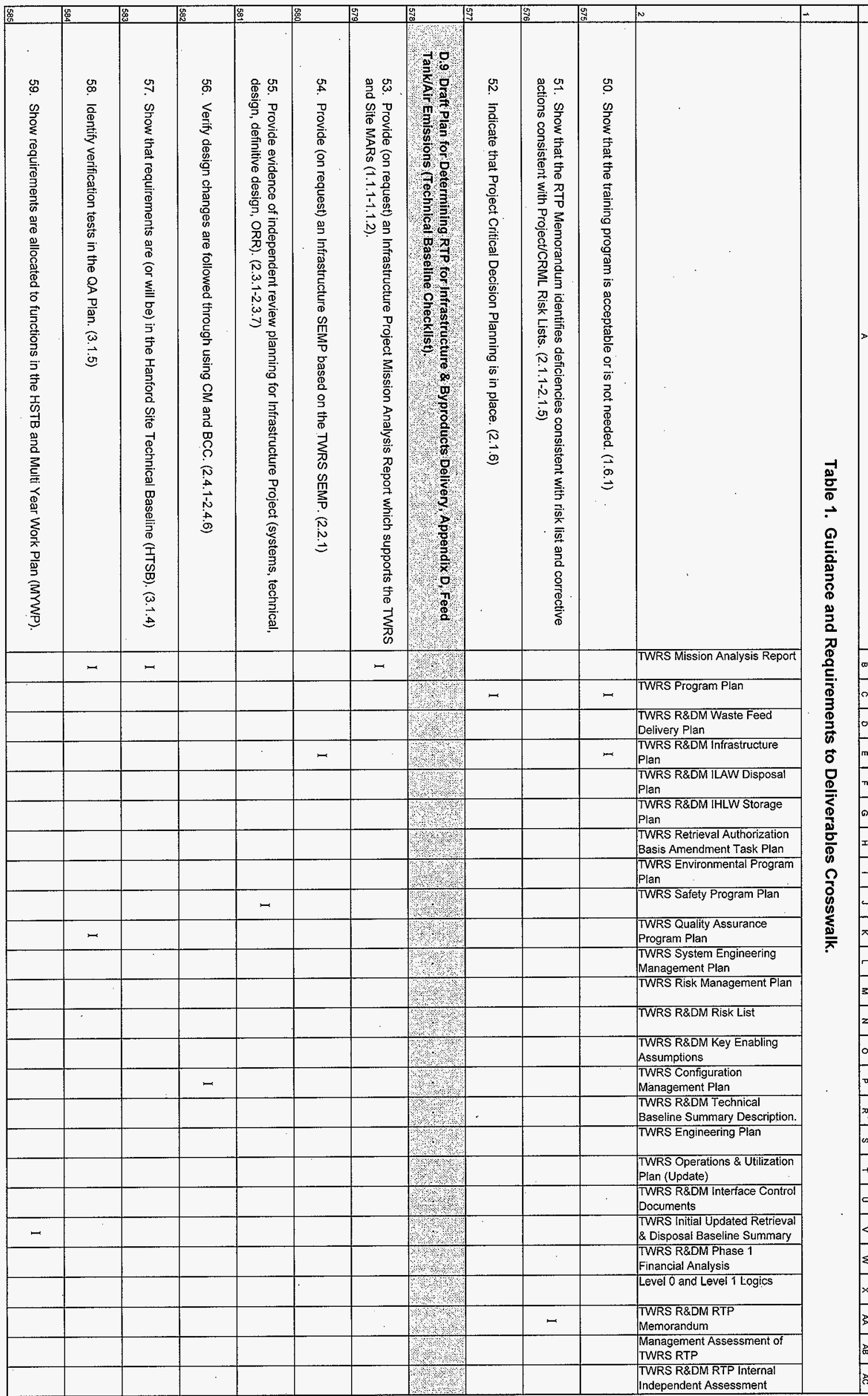




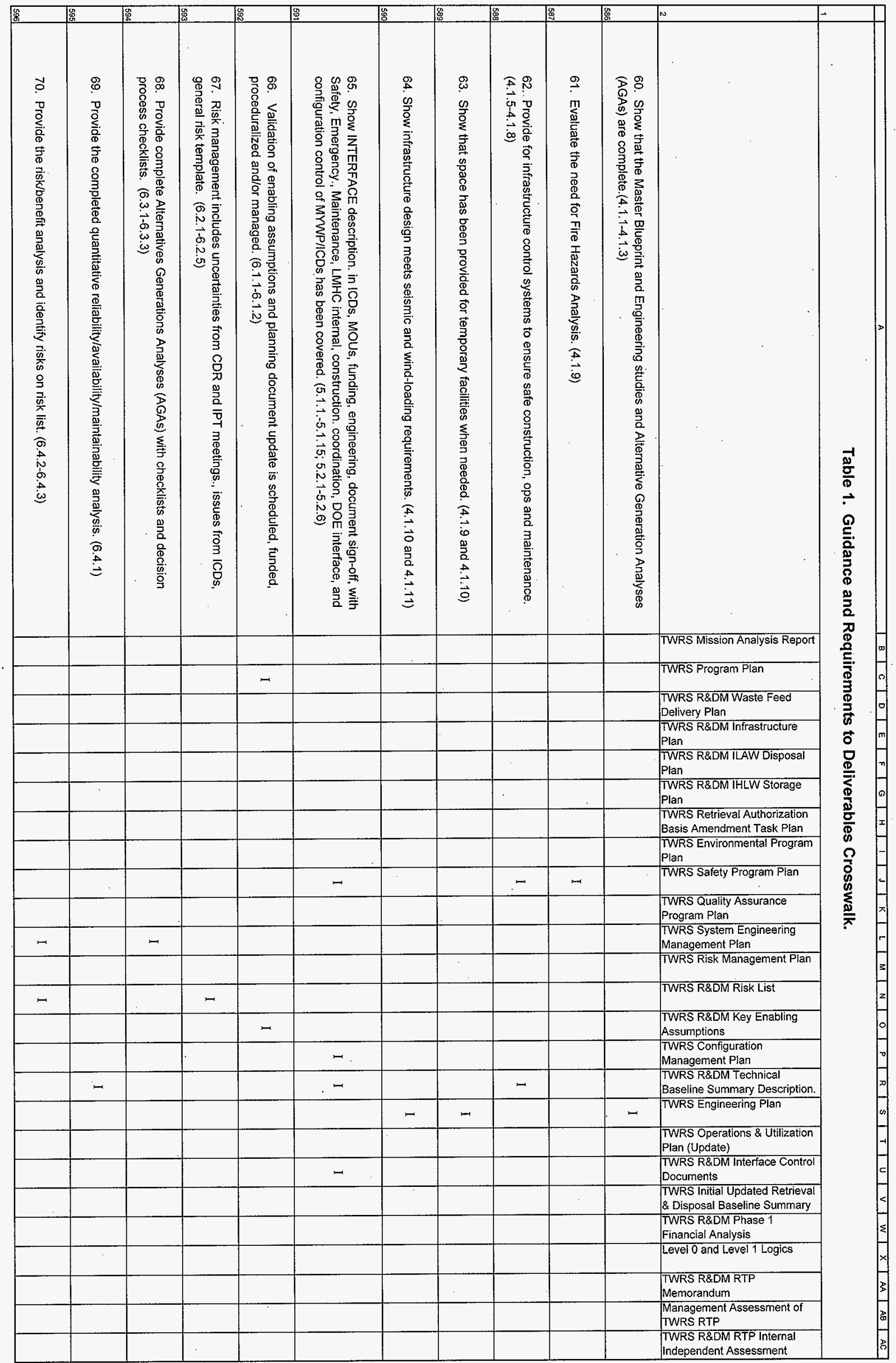




\begin{tabular}{|c|c|c|c|c|c|c|c|c|c|c|c|c|}
\hline 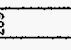 & 3 & 8 & 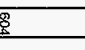 & & $\frac{8}{3}$ & 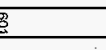 & & 8 & 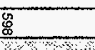 & 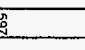 & $\omega$ & $\rightarrow$ \\
\hline 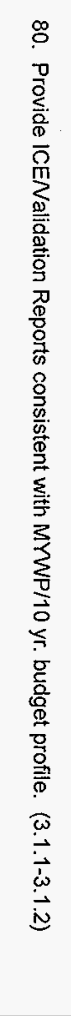 & 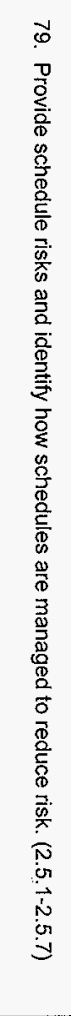 & 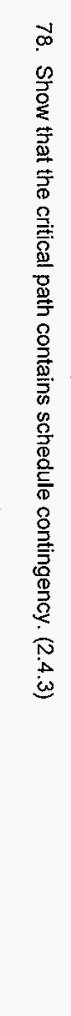 & 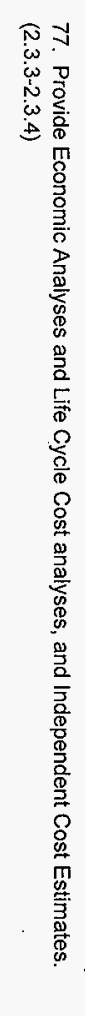 & 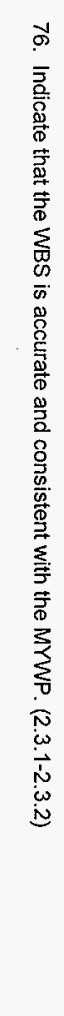 & 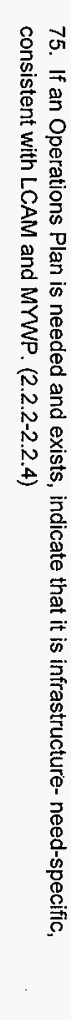 & 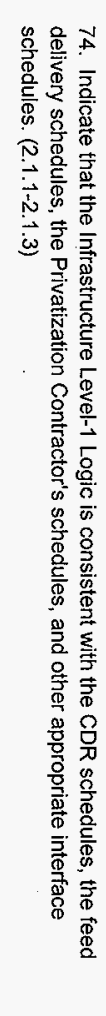 & 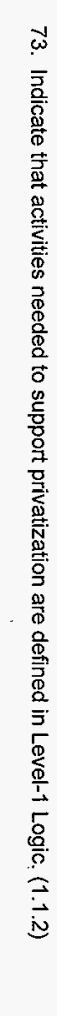 & 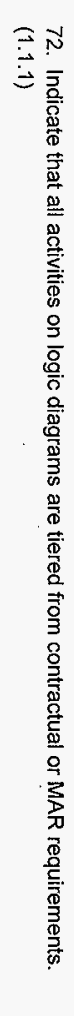 & 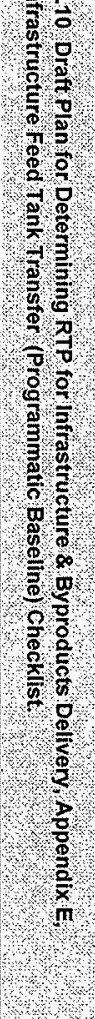 & 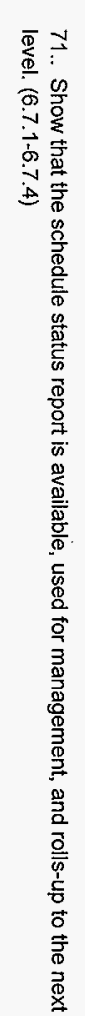 & . & 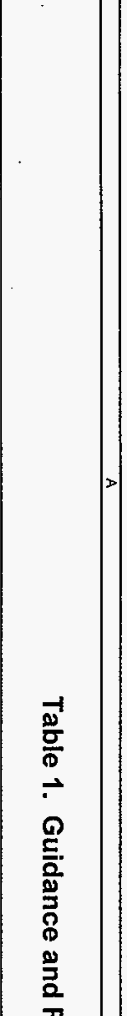 \\
\hline & & & & & & & & - & & & TWRS Mission Analysis Report & $\frac{1}{8}$ \\
\hline & - & & & & & & & & 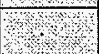 & - & TWRS Program Plan & 产 \\
\hline & & & & & & & & & & & $\begin{array}{l}\text { TWRS R\&DM Waste Feed } \\
\text { Delivery Plan }\end{array}$ & 苞 \\
\hline & & & & & & & & & & & $\begin{array}{l}\text { TWRS R\&DM Infrastructure } \\
\text { Plan }\end{array}$ & $\frac{\infty}{0}$ \\
\hline & & & & & & & & & & & $\begin{array}{l}\text { TWRS R\&DM ILAW Disposal } \\
\text { Plan }\end{array}$ & $\underset{\mathscr{\Phi}}{=}$ \\
\hline & & & & & & & & & 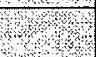 & . & $\begin{array}{l}\text { TWRS R\&DM IHLW Storage } \\
\text { Plan }\end{array}$ & হై \\
\hline & & & & & & & & & & & $\begin{array}{l}\text { TWRS Retrieval Authorization } \\
\text { Basis Amendment Task Plan }\end{array}$ & 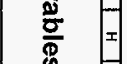 \\
\hline & & & & & & & & & & & $\begin{array}{l}\text { TWRS Environmental Program } \\
\text { Plan }\end{array}$ & 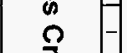 \\
\hline & & & & & & & & & & & TWRS Safety Program Plan & 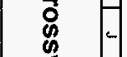 \\
\hline & & & & & & & & & & & $\begin{array}{l}\text { TWRS Quality Assurance } \\
\text { Program Plan }\end{array}$ & $\frac{\xi}{x}$ \\
\hline & & & & & & & & & & & $\begin{array}{l}\text { TWRS System Engineering } \\
\text { Management Plan }\end{array}$ & \\
\hline & $-m$ & & & & & & & & & & TWRS Risk Management Plan & \\
\hline & $m$ & & & & & & & & & & \begin{tabular}{|l} 
TWRS R\&DM Risk List \\
\end{tabular} & \\
\hline & - & & & & & & & & & & $\begin{array}{l}\text { TWRSR R\&DM Key Enabling } \\
\text { Assumptions }\end{array}$ & \\
\hline & & & & & - & & & & & & $\begin{array}{l}\text { WTSS Configuration } \\
\text { Management Plan }\end{array}$ & \\
\hline & & & & & & & & & & & $\begin{array}{l}\text { TWRS R\&DM Technical } \\
\text { Baseline Summary Description. }\end{array}$ & \\
\hline & & & & & & & & & & & TWRS Engineering Plan & \\
\hline & & & & & & & & & & & $\begin{array}{l}\text { TWRS Operations \& Utilization } \\
\text { Plan (Update) }\end{array}$ & \\
\hline & & & & & & & & & & & $\begin{array}{l}\text { TWRS RRDM Interface Control } \\
\text { Documents }\end{array}$ & \\
\hline & & - & & - & & - & & & & & $\begin{array}{l}\text { TWRS Initial Updated Retrieval } \\
\text { \& Disposal Baseline Summary }\end{array}$ & \\
\hline & - & & - & & & & & & & & $\begin{array}{l}\text { Tinancial Analysis } \\
\text { Fis }\end{array}$ & \\
\hline & & & & & & - & - & - & & & Level 0 and Level 1 Logics & \\
\hline & & & & & & & & & & & $\begin{array}{l}\text { TWRS R2DM RTP } \\
\text { Memorandum }\end{array}$ & \\
\hline & & & & & & & & & & & $\begin{array}{l}\text { Management Assessment of } \\
\text { TWRS RTP }\end{array}$ & \\
\hline - & & & - & & & & & & & & $\begin{array}{l}\text { TWRS R\&DM RTP Internal } \\
\text { Independent Assessment }\end{array}$ & \\
\hline
\end{tabular}




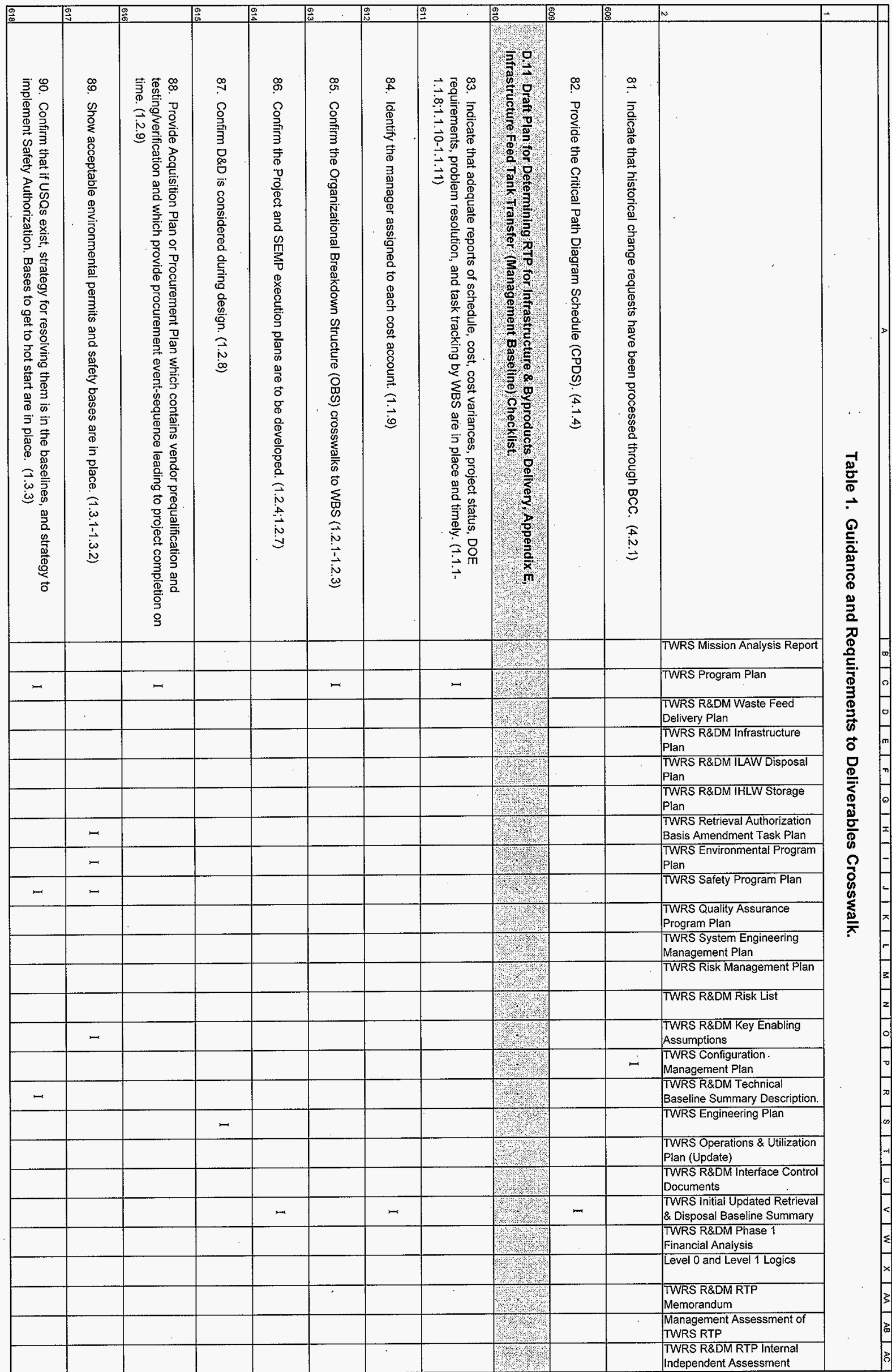




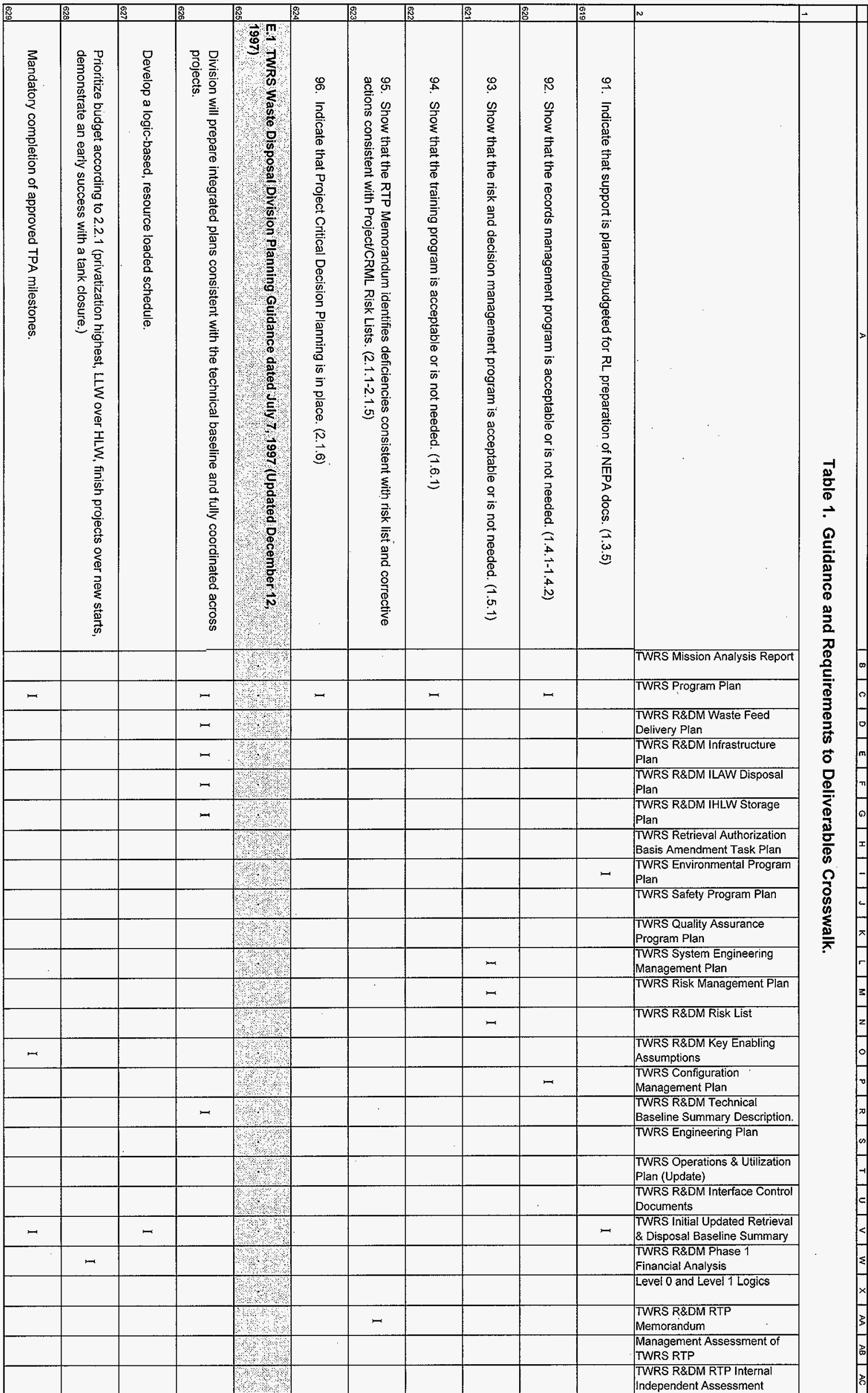




\begin{tabular}{|c|c|c|c|c|c|c|c|c|c|c|c|}
\hline- & 慝 & & & & $\underline{\underline{s}}$ & & 8 & 2 & & $\omega$ & F \\
\hline \multirow[t]{22}{*}{ 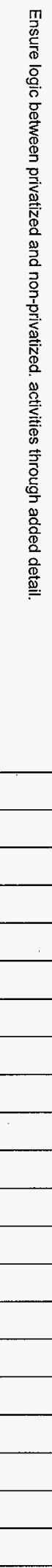 } & 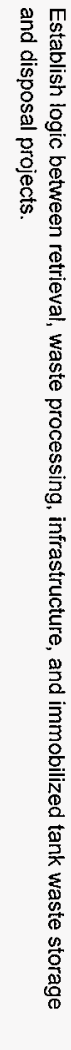 & 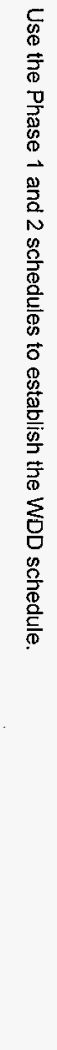 & 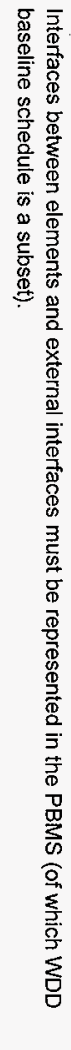 & 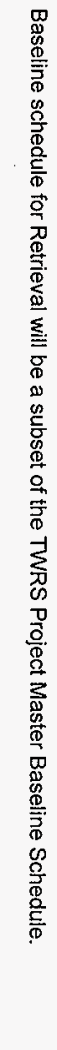 & 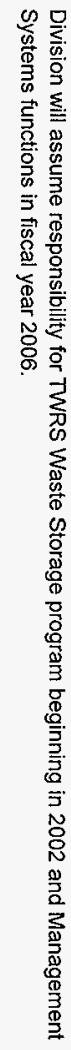 & 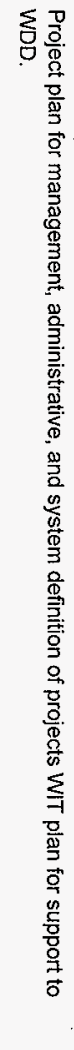 & 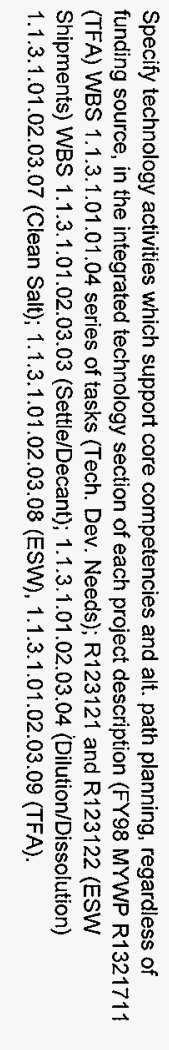 & 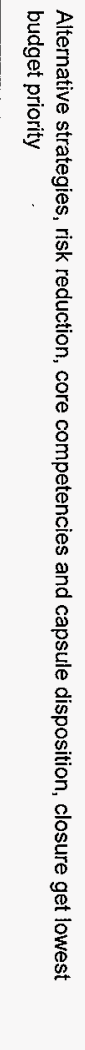 & 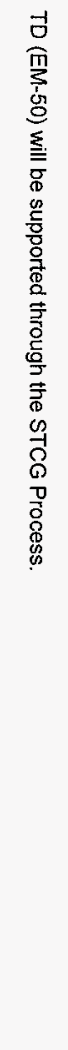 & & \multirow{26}{*}{ 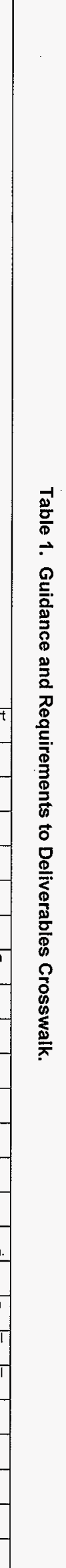 } \\
\hline & & & & & & & & & & TWRS Mission Analysis Report & \\
\hline & & - & - & 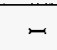 & & & & & - & TWRS Program Plan & \\
\hline & & & & & & & & & & $\begin{array}{l}\text { TWRS R\&DM Waste Feed } \\
\text { Delivery Plan }\end{array}$ & \\
\hline & & & & & & & & & & $\begin{array}{l}\text { FWRS R\&DM Infrastructure } \\
\text { Plan }\end{array}$ & \\
\hline & & & & & & & & & & $\begin{array}{l}\text { TWRS R\&DM ILAW Disposal } \\
\text { Plan }\end{array}$ & \\
\hline & & & & & & & & & & $\begin{array}{l}\text { TWRS R\&DM IHLW Storage } \\
\text { Plan }\end{array}$ & \\
\hline & & & & & & & & & & $\begin{array}{l}\text { TWRS Retrieval Authorization } \\
\text { Basis Amendment Task Plan }\end{array}$ & \\
\hline & & & & & & & & & & $\begin{array}{l}\text { TWRS Environmental Program } \\
\text { Plan }\end{array}$ & \\
\hline & & & & & & & & & & TWRS Safety Program Plan & \\
\hline & & & & & & & & & & $\begin{array}{l}\text { TWRS Quality Assurance } \\
\text { Program Plan }\end{array}$ & \\
\hline & & & & & & - & & & & $\begin{array}{l}\text { TWRS System Engineering } \\
\text { Management Plan }\end{array}$ & \\
\hline & & & & & & & & & & TWRS Rișk Management Plan & \\
\hline & & & & & & & & & & TWRS R\&DM Risk List & \\
\hline & & & & & - & & & & & $\begin{array}{l}\text { TWRS RRDM Key Enabling } \\
\text { Assumptions }\end{array}$ & \\
\hline & & & & & & & & & & \begin{tabular}{|l} 
TWRS Configuration \\
Management Plan
\end{tabular} & \\
\hline & & & & & & & & & & $\begin{array}{l}\text { TWRS R\&DM Technical } \\
\text { Base ine Summary Description. }\end{array}$ & \\
\hline & & & & & & . & & & & TWRS Engineering Plan & \\
\hline & & & & & & & & & & $\begin{array}{l}\text { TWRS Operations \& Utilization } \\
\text { Plan (Update) }\end{array}$ & \\
\hline & & & $\pi$ & & & & & & & $\begin{array}{l}\text { TWRS R\&DM Interface Control } \\
\text { Documents }\end{array}$ & \\
\hline & & - & - & - & & & & & & $\begin{array}{l}\text { TWRS Initial Updated Retrieval } \\
\text { \& Disposal Baseline Summary }\end{array}$ & \\
\hline & & & & & & & & - & & $\begin{array}{l}\text { TWRSR R2DM Phase } 1 \\
\text { Financial Analysis }\end{array}$ & \\
\hline \multirow[t]{4}{*}{-} & - & & & & & & & & & Level 0 and Level 1 Logics & \\
\hline & & & & & & & & & & $\begin{array}{l}\text { TWRS R\&DM RTP } \\
\text { Memorandum }\end{array}$ & \\
\hline & & & & & & & & & & $\begin{array}{l}\text { Management Assessment of } \\
\text { TWRS RTP }\end{array}$ & \\
\hline & & & & & & & & & & $\begin{array}{l}\text { TWRS R\&DM RTP Internal } \\
\text { Independent Assessment }\end{array}$ & \\
\hline
\end{tabular}




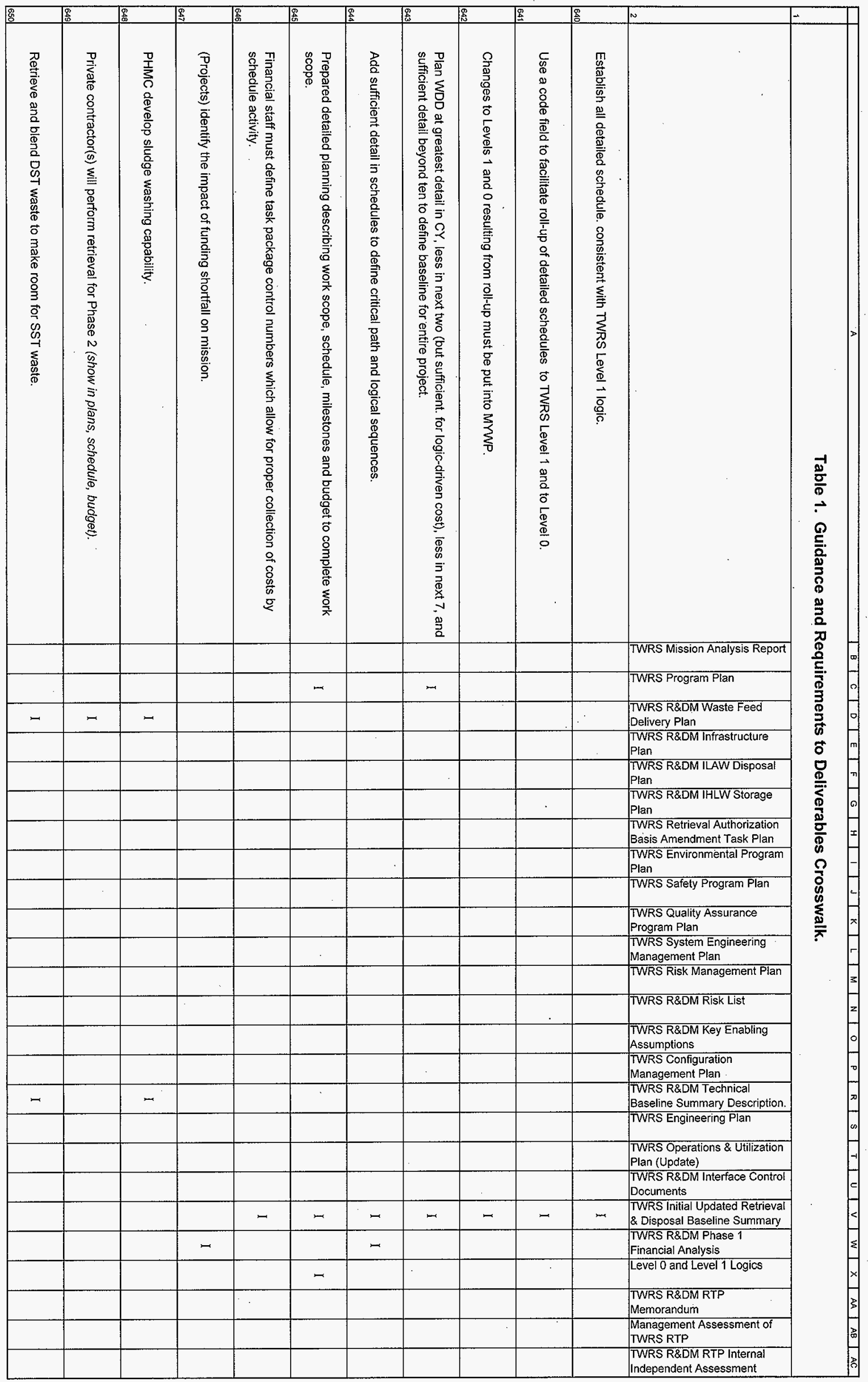




\begin{tabular}{|c|c|c|c|c|c|c|c|c|c|c|c|c|}
\hline & 曼 & & $\begin{array}{l}\underbrace{2}_{2} \\
\end{array}$ & & & & & 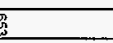 & & & $\omega$ & \\
\hline \multirow[t]{16}{*}{ 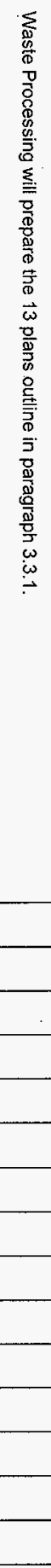 } & 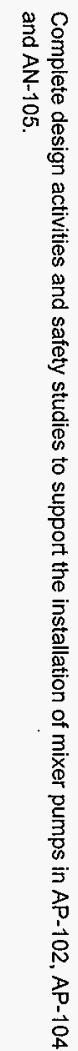 & 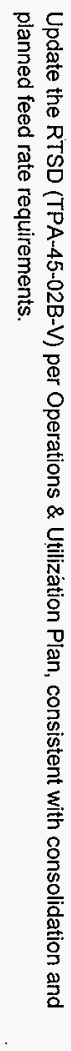 & 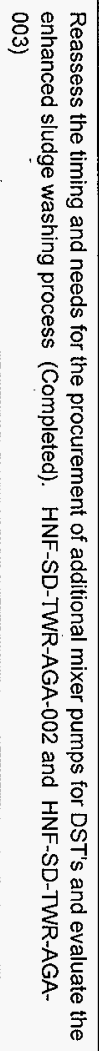 & 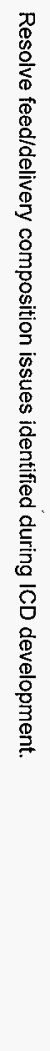 & 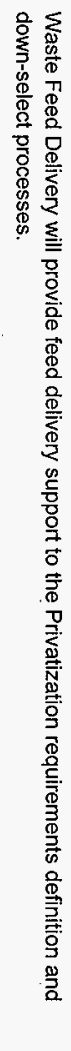 & 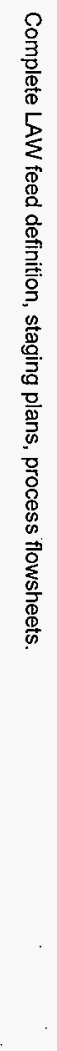 & 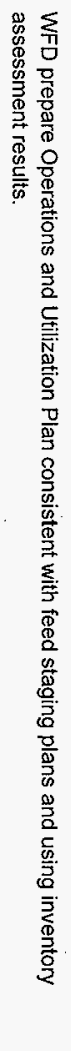 & 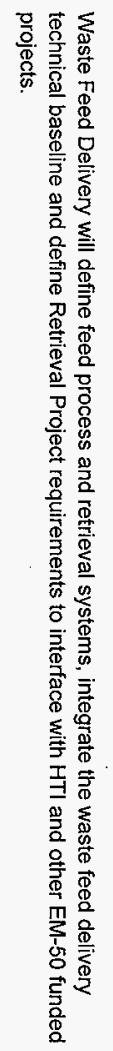 & 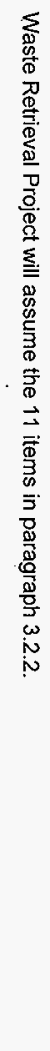 & 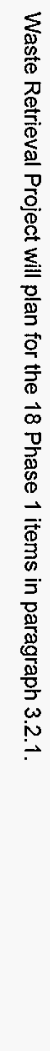 & & 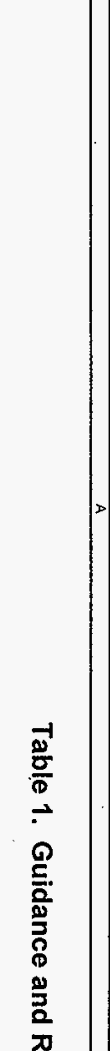 \\
\hline & & - & & & & & & & & & TWRS Mission Analysis Report & 总 \\
\hline & & & & & & & & & & & TWRS Program Plan & 焉 \\
\hline & & & & - & - & & & - & - & - & $\begin{array}{l}\text { TWRS R\&DM Waste Feed } \\
\text { Delivery Plan }\end{array}$ & 㟧 \\
\hline & & & & & & & & & & & $\begin{array}{l}\text { TwRS R\&DM Infrastructure } \\
\text { Plan }\end{array}$ & $\frac{0}{0}$ \\
\hline & & & & & & & & & & & $\begin{array}{l}\text { TWRS R\&DM ILAW Disposal } \\
\text { Plan }\end{array}$ & $\underset{\square}{\underline{\Phi}}$ \\
\hline & & & & & & & & & & & $\begin{array}{l}\text { TWRRS R\&DM IHLW Storage } \\
\text { Plan }\end{array}$ & శె' \\
\hline & . & & & & & & & & & & \begin{tabular}{|l|} 
TWRS Retrieval Authorization \\
Basis Amendment Task Plan
\end{tabular} & $\frac{\frac{0}{0}}{D}$ \\
\hline & r & & & & & & & & & & $\begin{array}{l}\text { WWRS Environmental Program } \\
\text { Plan }\end{array}$ & 足 \\
\hline & r & & & & & & & & & & TWRS Safety Program Plan & 实 \\
\hline & & & & & & & & & & & $\begin{array}{l}\text { TWRS Quality Assurance } \\
\text { Program Plan }\end{array}$ & 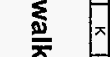 \\
\hline & & & & & & & & & & & $\begin{array}{l}\text { TWRS System Engineering } \\
\text { Management Plan }\end{array}$ & \\
\hline & & & & & & & & & & & TWRS Risk Management Plan & \\
\hline & & & & & & & & & & & TWRS R\&DM Risk List & \\
\hline & & & & & & & & & & & $\begin{array}{l}\text { TWRS RRDM Key Enabling } \\
\text { Assumptions }\end{array}$ & \\
\hline & & & & & & & & & & & $\begin{array}{l}\text { TWRS Configuration } \\
\text { Management Plan }\end{array}$ & \\
\hline \multirow[t]{10}{*}{$\varpi$} & r & & & & & $m$ & & - & - & - & $\begin{array}{l}\text { TWRS R\&DM Technical } \\
\text { Baseline Summary Description. }\end{array}$ & \\
\hline & - & & & & & & & & & & TWRS Engineering Plan & \\
\hline & & - & & & & & - & & & & $\begin{array}{l}\text { TWRS Operations \& Utilization } \\
\text { Plan (Update) }\end{array}$ & \\
\hline & & & & - & - & & & & & & $\begin{array}{l}\text { TWRS R\&DM Interface Control } \\
\text { Documents }\end{array}$ & \\
\hline & & & & & & - & & - & - & - & \begin{tabular}{|l|} 
TWRS Initial Updated Retrieval \\
\& Disposal Baseline Summary
\end{tabular} & \\
\hline & & & & & & & & . & - & $\pi$ & $\begin{array}{l}\text { TWRS R2DM Phase } 1 \\
\text { Financial Analysis }\end{array}$ & \\
\hline & & & & & & & & & & & Level 0 and Level 1 Logics & \\
\hline & & & & & & & & & & & $\begin{array}{l}\text { TWRS R\&DM RTP } \\
\text { Memorandum. }\end{array}$ & \\
\hline & & & & & & & & & & & $\begin{array}{l}\text { Management Assessment of } \\
\text { TWWRS RTP }\end{array}$ & \\
\hline & & & & & & & & & & & \begin{tabular}{|l} 
TWRS R\&DM RTP Internal \\
Independent Assessment
\end{tabular} & \\
\hline
\end{tabular}




\begin{tabular}{|c|c|c|c|c|c|c|c|c|c|c|c|c|}
\hline 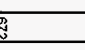 & 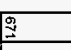 & 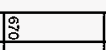 & 兽 & 盘 & 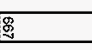 & 量 & 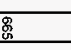 & 厚 & 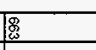 & 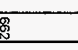 & N & - \\
\hline 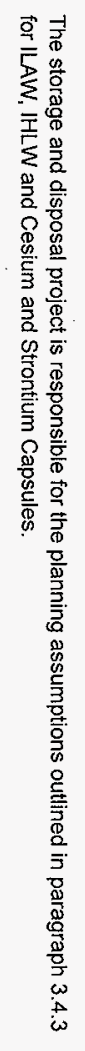 & 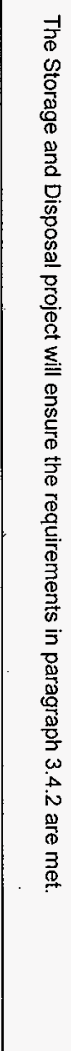 & 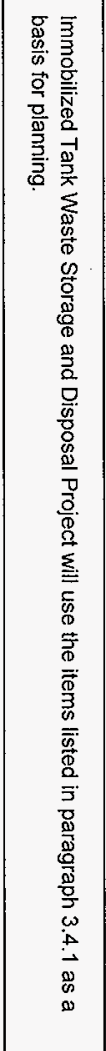 & 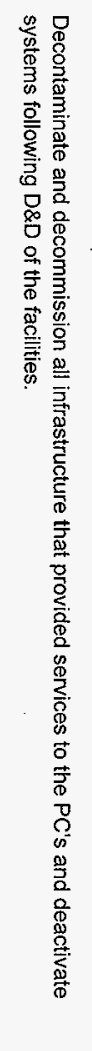 & 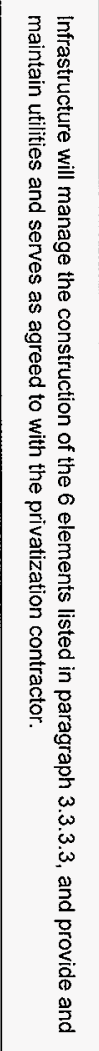 & 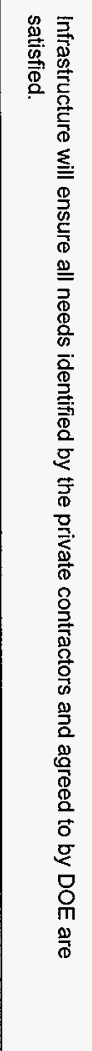 & 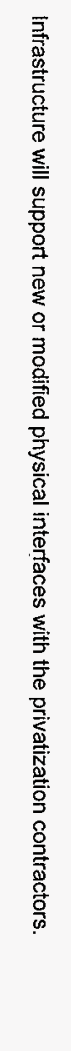 & 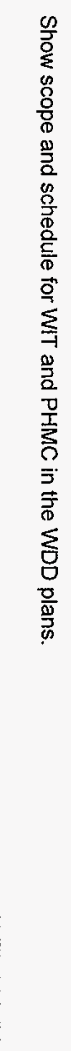 & 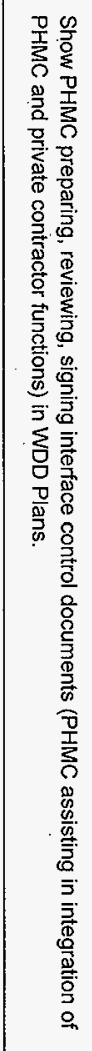 & 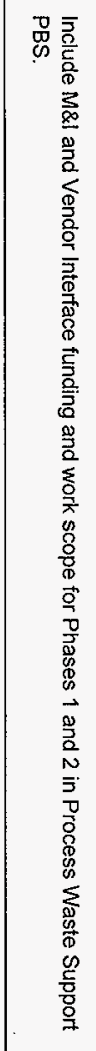 & 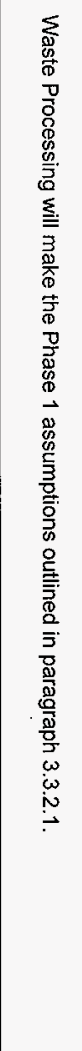 & & 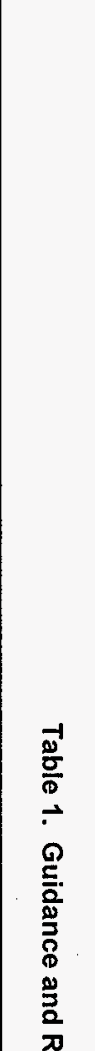 \\
\hline & & & & & & & & & & & TWRS Mission Analysis Report & 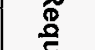 \\
\hline & & & & & & & - & 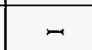 & & & TiWRS Program Plan & 商 \\
\hline & & & & & & & & & & & $\begin{array}{l}\text { WWRS R\&DM Waste Feed } \\
\text { Delivery Plan }\end{array}$ & $\stackrel{\underline{0}}{\rightleftarrows}$ \\
\hline & & & - & - & - & - & & & & & $\begin{array}{l}\text { TWRS R\&DM Infrastructure } \\
\text { Plan }\end{array}$ & $\frac{\infty}{0}$ \\
\hline- & & & & & & & & & & & $\begin{array}{l}\text { TWRS R\&DM ILAW Disposal } \\
\text { Plan }\end{array}$ & $\underset{\underline{\Phi}}{=}$ \\
\hline - & & & & & & & & & & & $\begin{array}{l}\text { TWRS R\&DM IHLW Storage } \\
\text { Plan }\end{array}$ & $\underset{\Phi}{2}$ \\
\hline & & & & & & & & & & & $\begin{array}{l}\text { TWRS Retrieval Authorization } \\
\text { Basis Amendment Task Plan }\end{array}$ & $\frac{\mathrm{\sigma}}{0}$ \\
\hline & - & & & & & & & & & & $\begin{array}{l}\text { TWRS Environmental Program } \\
\text { Plan }\end{array}$ & $\stackrel{n}{\Omega}$ \\
\hline & - & & & & & & & & & & TWRS Safety Program Plan & : \\
\hline & & & & & & & & & & & $\begin{array}{l}\text { TWRS Quality Assurance } \\
\text { Program Plan }\end{array}$ & $\underset{ٍ}{\stackrel{\Sigma}{*}}$ \\
\hline & & & & & & & & & & & $\begin{array}{l}\text { TWRS System Engineering } \\
\text { Management Plan }\end{array}$ & \\
\hline & & & & & & & & & & & TWRS Risk Management Plan & \\
\hline & & & . & & & & & & & & TWRS R\&DM Risk List & \\
\hline & & & & & & & & & & - & $\begin{array}{l}\text { TWRS R\&DM Key Enabling } \\
\text { Assumptios }\end{array}$ & \\
\hline & & & & & & & & & & & $\begin{array}{l}\text { TWRS Configuration } \\
\text { Management Plan }\end{array}$ & \\
\hline- & - & $m$ & $\pi$ & & & & & & & - & $\begin{array}{l}\text { TWRS REDM Technical } \\
\text { Baseline Summary Description. }\end{array}$ & \\
\hline & & & & & & & & & & & TWRS Engineering Plan & \\
\hline & & & & & & & & & & & $\begin{array}{l}\text { TWRS Operations \& Utilization } \\
\text { Plan (Update) }\end{array}$ & \\
\hline & & & - & - & - & - & & - & & & $\begin{array}{l}\text { TWRS R\&DM Interface Control } \\
\text { Documents }\end{array}$ & \\
\hline & & & & & & & - & & - & & $\begin{array}{l}\text { TWRS Initial Updated Retrieval } \\
\text { \& Disposal Baseline Summary }\end{array}$ & \\
\hline & & & & & & & & & & & $\begin{array}{l}\text { TWRS R\&DM Phase } 1 \\
\text { Financial Analysis }\end{array}$ & \\
\hline & & & & & & & & & & & Level 0 and Level 1 Logics & \\
\hline & & & & & & & & & & & $\begin{array}{l}\text { TWRS R\&DM RTP } \\
\text { Memorandum }\end{array}$ & \\
\hline & & & & & & & & & & & $\begin{array}{l}\text { Management Assessment of } \\
\text { TWRS RTP }\end{array}$ & \\
\hline & & & & & & & & & & & $\begin{array}{l}\text { TWRS R2DM RTP Internal } \\
\text { independent Assessment }\end{array}$ & \\
\hline
\end{tabular}




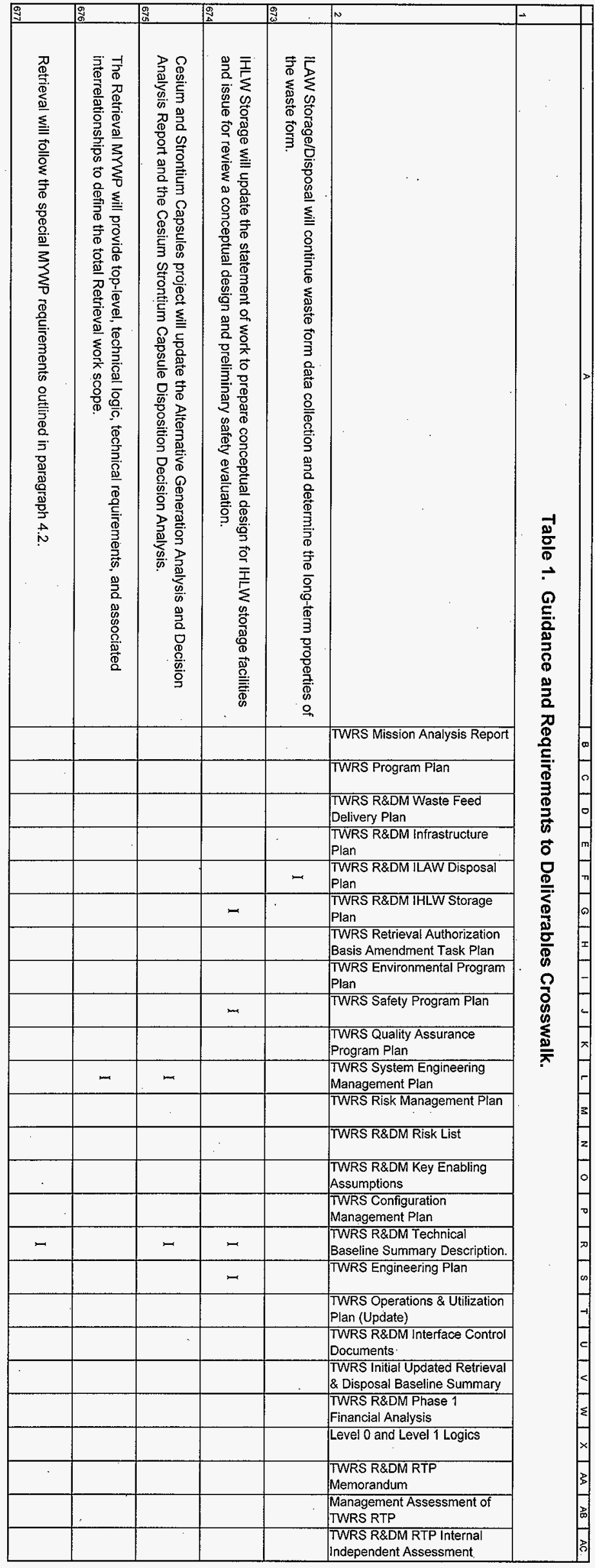




\section{DISTRIBUTION SHEET}

\begin{tabular}{|c|c|c|c|c|c|}
\hline \multirow{2}{*}{ Document Control Servic } & \multirow{2}{*}{\multicolumn{3}{|c|}{$\begin{array}{l}\text { From } \\
\text { TWRS Configuration Management }\end{array}$}} & \multicolumn{2}{|l|}{ Päge: $1-0 f i$} \\
\hline & & & & \multicolumn{2}{|c|}{ Date January 3, 1998} \\
\hline \multirow{2}{*}{\multicolumn{4}{|c|}{$\begin{array}{l}\text { Project Title/work Order. } \\
\text { Tank Waste Remediation Systems (HNF-2020) }\end{array}$}} & \multicolumn{2}{|l|}{ EDT No. } \\
\hline & & & & \multicolumn{2}{|c|}{ ECN No. 646202} \\
\hline Name & MSIN & $\begin{array}{c}\text { Text } \\
\text { With All } \\
\text { Attach. }\end{array}$ & $\begin{array}{l}\text { Text } \\
\text { Only }\end{array}$ & $\begin{array}{c}\text { Attach.! } \\
\text { Appendix } \\
\text { Oniy }\end{array}$ & $\begin{array}{c}\text { EDT/ECN } \\
\text { Only }\end{array}$ \\
\hline
\end{tabular}

E. Alford

J.N. ATibert

H.L. Boston

M.P. Delozier

O.A. Halvorson

J.0. Honeýmian

K.N. Jordan

M.R. Lewis

D.M. McDaniel

R.j. Murkowski

S.M. O'Toole

M.A. Payne

L.G. Peck

M.L. Peterman

R.W. Powell

R.P. Raven

R.E, Raymond

S.H. Rifaey

B. Root

P.S. Schaus

S.E. Seeman .

W.R. Swita

R.L. Treat

J.M. Vann

J.H. Wicks Jr

R.D. Wojtasek
H7-06 $X$

S2-48

G3-21

R2-58

G3-21

G3-21

G3-21

H5- 03

H7 -06

H6-37

G3-21

R2-58

H6-35

H7- 06

H5-03.

R1-51

$R 2-38$

R1-56

G3-21

H5- 03

H6-35

H5- 03

H5- 03

H6-37

H7-07

G3-21 $x$
$x$

$x$

$x$

$x$

$x$

$x$

$x$

$x$

$x$

$x$

$x$

$x$

$x$

$x$

$x$

$x$

$x$

$x$

$x$

$x$

$x$

$x$

$x$

$x$

$x$

Central Files

B1-07 X X 